\title{
A MULTICULTURAL NUTRITION AND CULINARY INTERVENTION FOR MIDDLE SCHOOL STUDENTS: PINK AND DUDE CHEFS, PHASE 2
}

\author{
A Thesis \\ presented to \\ the Faculty of California Polytechnic State University, \\ San Luis Obispo
}

In Partial Fulfillment

of the Requirements for the Degree

Master of Science in Agriculture,

with specialization in Food Science and Nutrition

by

Jaime Lynn Lockhart

August 2014 
(C) 2014

Jaime Lynn Lockhart

ALL RIGHTS RESERVED 


\section{COMMITTEE MEMBERSHIP}

TITLE:

AUTHOR:

DATE SUBMITTED:

COMMITTEE CHAIR:

COMMITTEE MEMBER:

COMMITTEE MEMBER:
A Multicultural Nutrition and Culinary Intervention for Middle School Students: Pink and Dude Chefs, Phase 2

Jaime Lynn Lockhart

August 2014

Lisa Nicholson, Ph.D., RD, Associate Professor, Food Science and Nutrition Department

Aydin Nazmi, Ph.D., Associate Professor, Food Science and Nutrition Department, Center for Solutions Through Research in Diet and Exercise (STRIDE)

Arlene Grant-Holcomb, Ed.D., RD, Lecturer, Food Science and Nutrition Department 


\begin{abstract}
A Multicultural Nutrition and Culinary Intervention for Middle School Students: Pink and Dude Chefs, Phase 2

Jaime Lynn Lockhart
\end{abstract}

The prevalence of adolescent obesity in the United States has more than quadrupled in the past 30 years, growing from 5\% to nearly $21 \%$ in $12-19$ year olds. Although obesity has many interrelated causes, a lack of knowledge and practical skill to prepare healthy food from scratch is an important factor. Research indicates that nutrition and culinary interventions based on behavioral change theories may improve mediators of healthful eating in adolescents.

Pink and Dude Chefs, Phase 2, an eight-week after-school nutrition and culinary intervention targeted towards middle school students in a predominantly low-income, Hispanic community, was developed and evaluated. The curriculum called "Around the World" was designed to enhance knowledge and cooking skills through a multicultural approach. Participants learned about the food culture and customs of a different country each week and then created a recipe of that country from scratch. Research assistants from California Polytechnic State University implemented the lessons. The program took place at Mesa Middle School in Arroyo Grande, CA over two consecutive academic quarters, Fall 2013 and Winter 2014. Participants were 16 seventhand eighth-grade students aged 12-14. The program consisted of 8 lessons that were divided into a classroom-based learning portion and a kitchen-based cooking portion. A pre- and post-intervention survey was developed to assess change in self-efficacy for cooking, cooking skills, nutrition knowledge, outcome expectancies, and perceived social and family norms.

Paired t-tests indicated that participants demonstrated a significant increase in nutrition knowledge $(P<0.0001)$ and cooking skills $(P=0.02)$ after participation in Pink and Dude Chefs, Phase 2. Empowering youth by improving food-related knowledge and skills is an important step toward improving the health of our nation. Further research is needed to examine how nutrition and culinary interventions may impact long-term health promoting behaviors. 


\section{ACKNOWLEDGMENTS}

First I would like to thank my committee chair and advisor, Dr. Lisa Nicholson, for her continual encouragement and guidance. It has been a true privilege to collaborate with such a knowledgeable scholar. I would like to thank my committee member, Dr. Aydin Nazmi, for pushing me beyond my limits and offering constant support. I would also like to thank my committee member Dr. Arlene Grant-Holcomb for her enthusiasm and positivity.

I would like to extend a huge thank you to the entire Cal Poly STRIDE team. This project would not have been possible if not for each and every one of you, and for that I am truly grateful. To all of the Health Ambassador students and volunteers that made weekly lesson implementation possible, your work is very much appreciated. Thank you to the Food Bank Coalition of San Luis Obispo County for their generous donations of food ingredients to support my research. Thank you to the Maxwell Foundation for their generous support of STRIDE and Pink and Dude Chefs. Thank you to Ed and Elaine Reeves and the rest of the Bright Futures staff at Mesa Middle School for a successful partnership. I would also like to extend an extraordinary thank you to Tianna Sheehan for her constant mentorship and support.

Last but not least, I would like to thank my wonderful family for their endless support and love. None of this would have been possible without you. And to my fiancé Corbin Kembel, you have inspired me more than you know. Thank you for the encouragement, love, and laughter throughout this journey together. 


\section{TABLE OF CONTENTS}

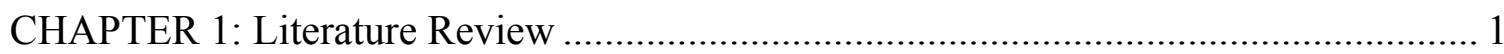

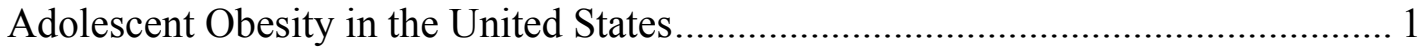

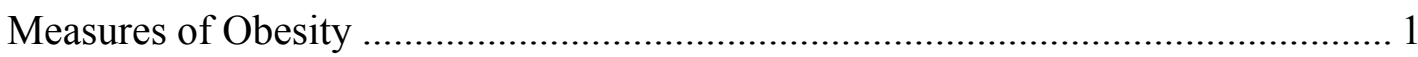

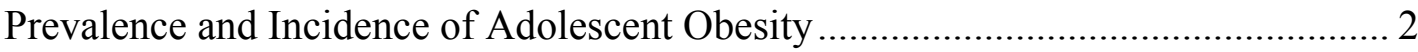

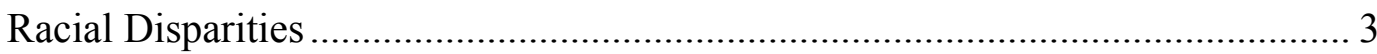

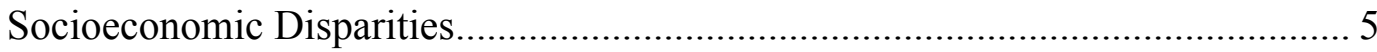

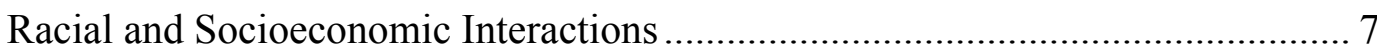

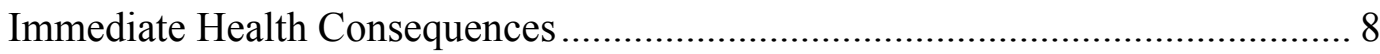

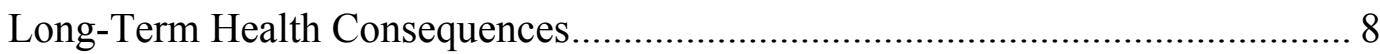

Psychological \& Social Consequences ............................................................. 9

Economic Consequences .............................................................................. 9

A Complex of Interrelated Factors Causing Adolescent Obesity ................................ 9

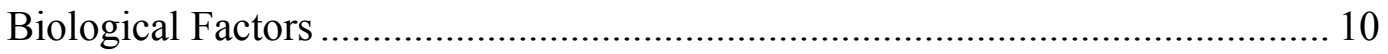

Social and Environmental Factors ............................................................... 12

Psychological and Behavioral Factors ........................................................... 13

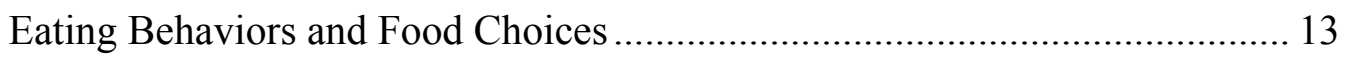

The Importance of Culinary Skills for Healthful Eating ......................................... 15

The Importance of Social and Family Norms for Healthful Eating.......................... 18

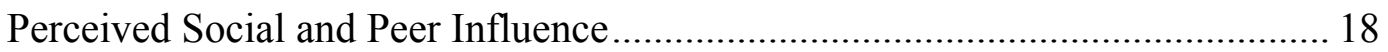

Perceived Family Norms and Influence............................................................. 19

Approaches to Prevent and Treat Adolescent Obesity ............................................. 20

Integrated Behavioral Model for Nutrition and Culinary Education ......................... 22

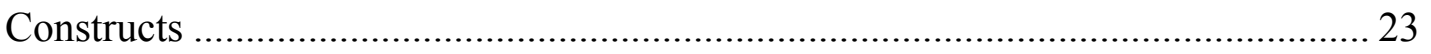

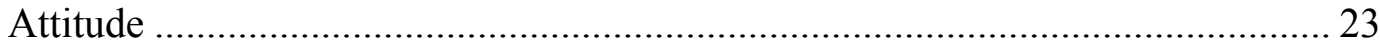

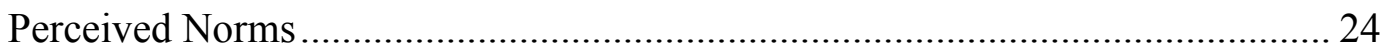

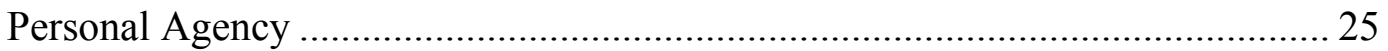

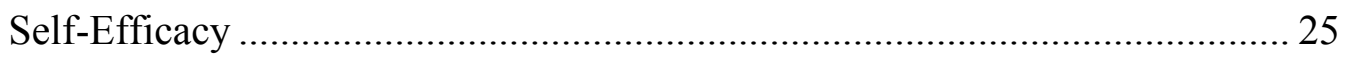

Obesity Prevention through Nutrition and Culinary Education................................ 26

Chef and Child Foundation Program .............................................................. 26

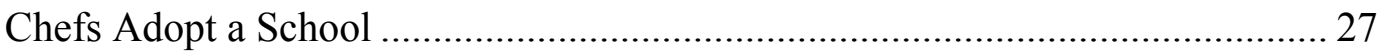

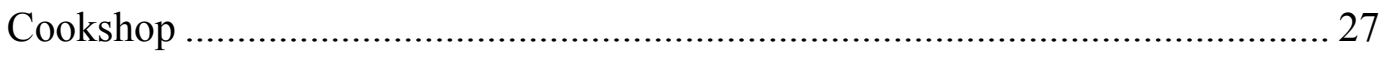

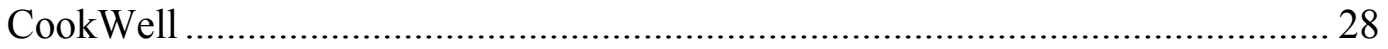

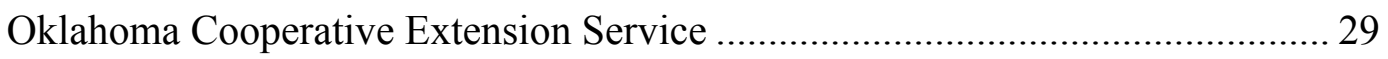

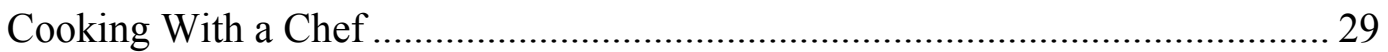

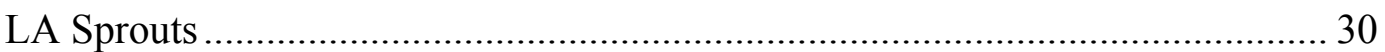

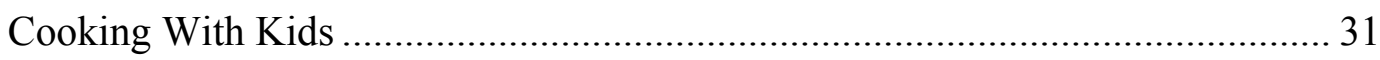

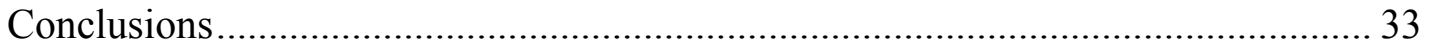




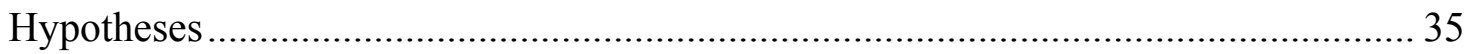

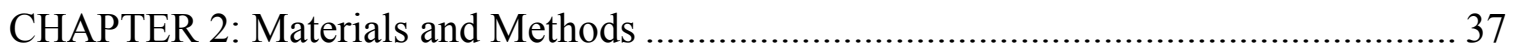

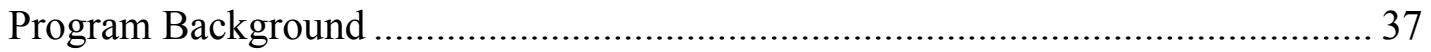

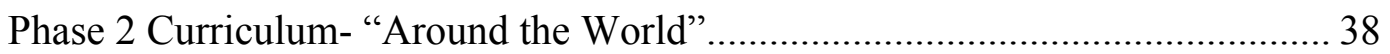

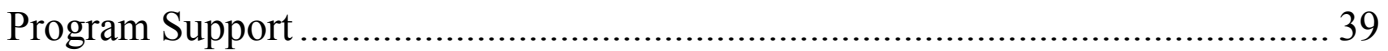

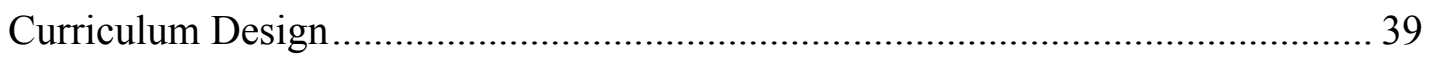

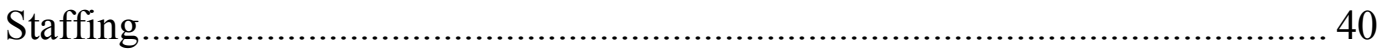

Weekly Lessons: Classroom Portion ................................................................. 41

Goal Sheets to Extend Learning Outside the Classroom .................................... 42

Weekly Lessons: Kitchen Portion...................................................................... 43

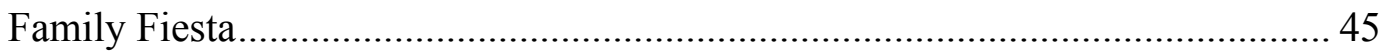

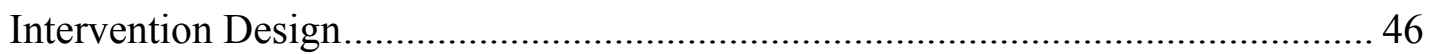

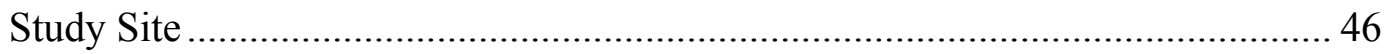

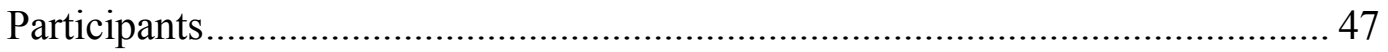

Institutional Review Board Process ............................................................ 48

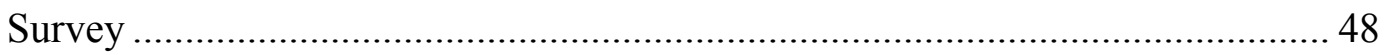

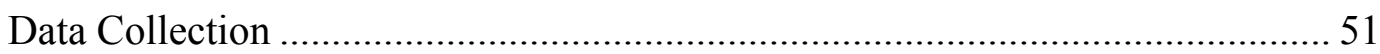

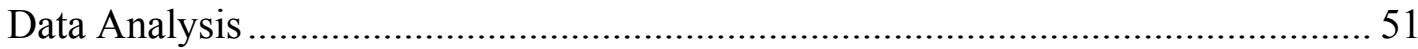

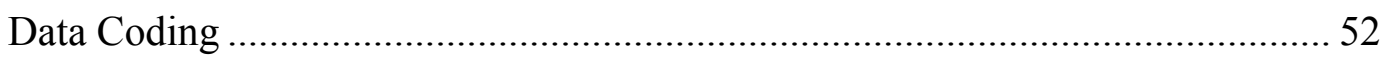

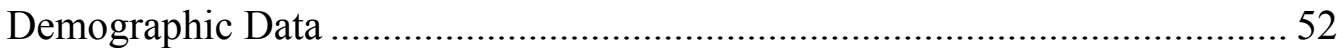

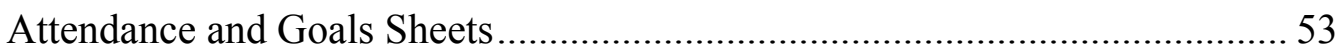

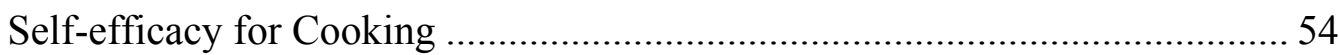

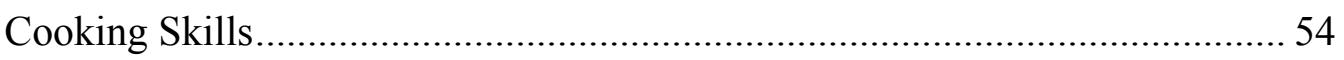

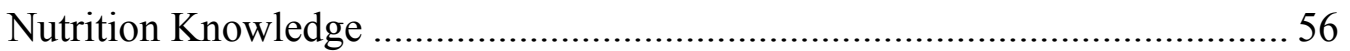

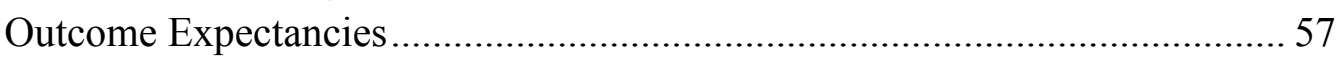

Perceived Social and Family Norms ............................................................ 58

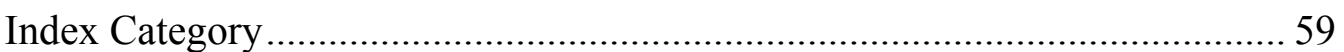

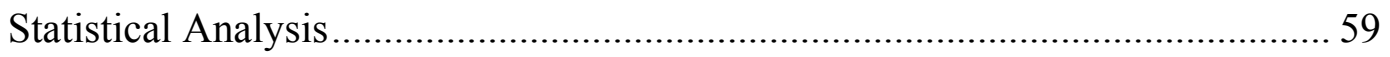

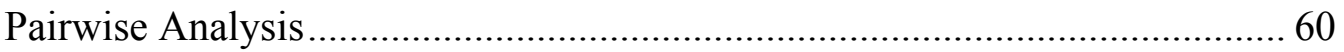

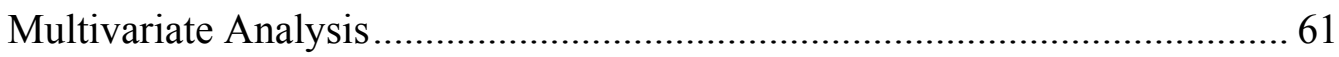

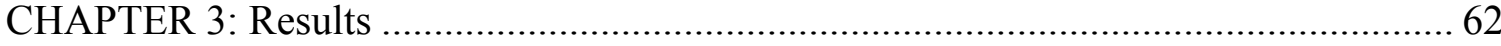

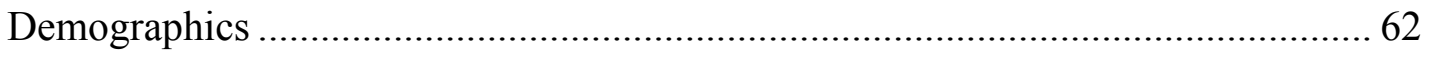

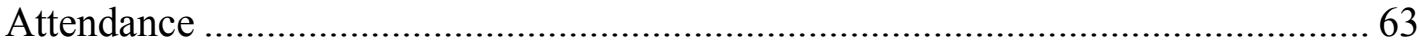

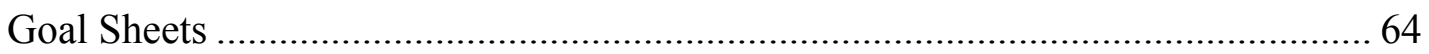

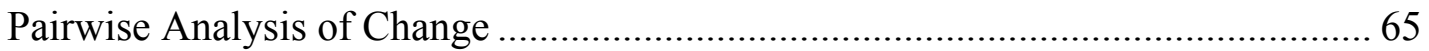

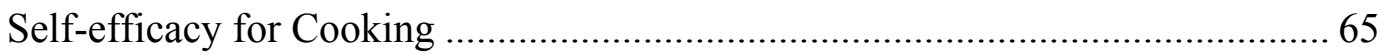

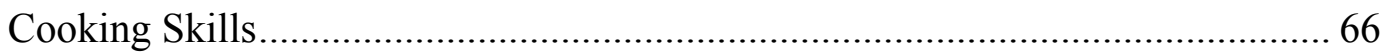

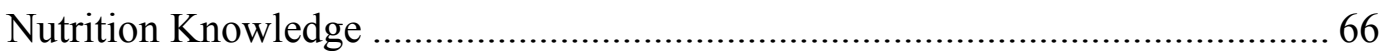

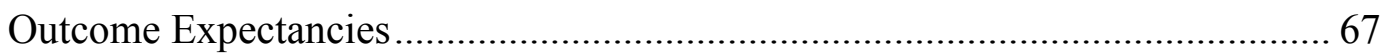

Perceived Social and Family Norms ................................................................... 68 


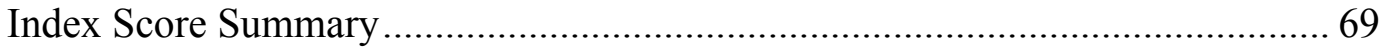

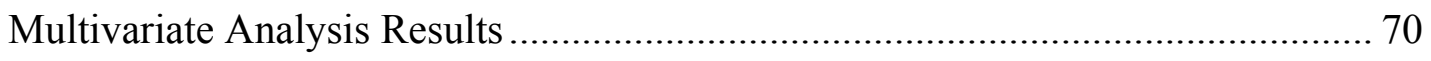

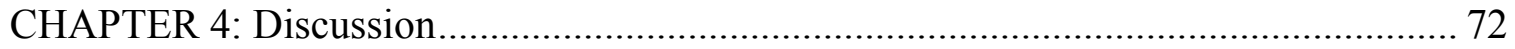

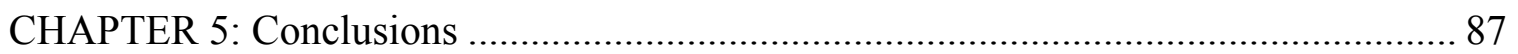

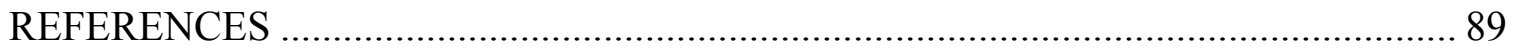

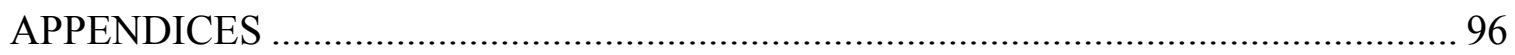

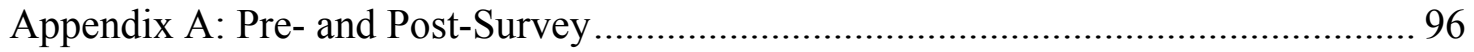

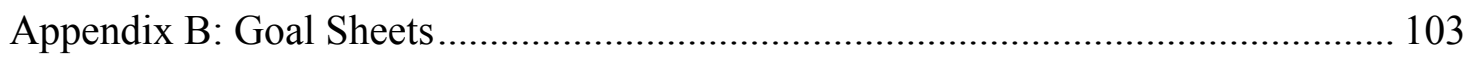

Appendix C: Recipes ....................................................................................... 107

Appendix D: Human Subjects Protocol Approval Form ......................................... 112

Appendix E: Human Subjects Research Protocol.................................................... 115

Appendix F: Informed Assent Form for Participant ............................................... 117

Appendix G: Informed Permission Form for Parents ............................................. 119 


\section{LIST OF TABLES}

Table 1.1: Weight status categories according to the CDC growth chart percentiles (National Institutes of Health, 2012). ..................................................................... 2

Table 2.1: Pink and Dude Chefs, Phase 2: Goals for the lesson........................................ 43

Table 2.2: Pink and Dude Chefs, Phase 2 recipe schedule............................................. 45

Table 2.3: Constructs and corresponding sample questions from pre- and post-

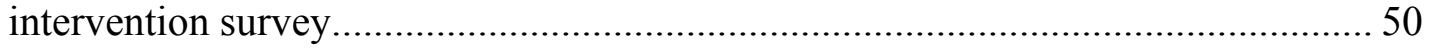

Table 2.4: Example coding of a survey question......................................................... 52

Table 2.5: Index categories and possible score ranges. ............................................... 59

Table 3.1: Baseline demographic characteristics of Pink and Dude Chefs

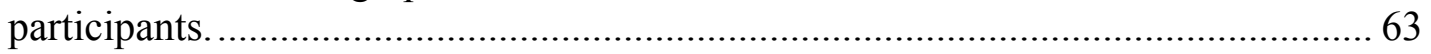

Table 3.2: Participant Attendance for Pink and Dude Chefs, Phase 2............................. 64

Table 3.3: Mean scores for self-efficacy for cooking and change in mean score

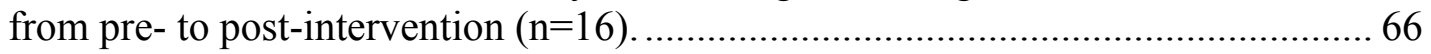

Table 3.4: Mean scores for cooking skills and change in mean score from pre-

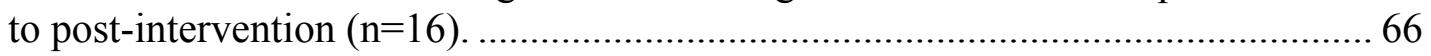

Table 3.5: Mean scores for cooking skills and change in mean score from pre- to post-intervention $(\mathrm{n}=16)$.

Table 3.6: Mean scores for outcome expectancies and change in mean score from

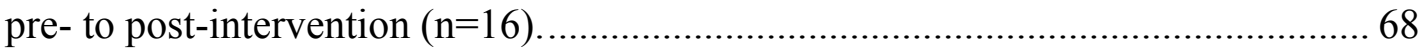

Table 3.7: Mean scores for perceived social and family norms and change in mean

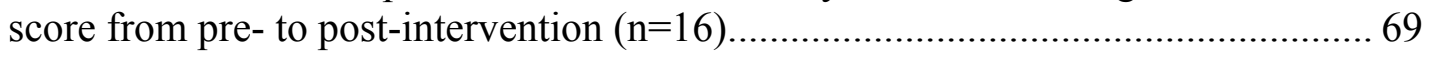

Table 3.8: Mean scores for index categories and change in mean index score from pre- to post-intervention $(\mathrm{n}=16)$. 70 


\section{LIST OF FIGURES}

Figure 1.1: Prevalence of adolescent obesity in non-Hispanic white and Hispanic adolescents aged 2-19, by sex and age (Ogden et al., 2012). ............................... 4

Figure 1.2: Biopsychosocial model of obesity (Skelton et al., 2006)........................... 10

Figure 1.3. The Integrated Behavioral Model (Glanz et al., 2008)............................... 23

Figure 2.1: Flowchart depicting the structure of the Pink and Dude Chefs program

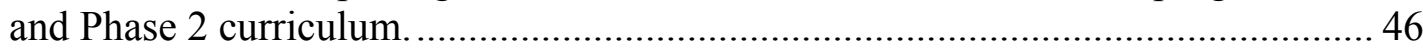

Figure 3.1: Total number of goal sheets submitted by participant. Column colors indicate the ratio of family goals to friend goals completed per individual. 65

Figure 3.2: Change in mean index scores from pre- to post-intervention. ..................... 70 


\section{CHAPTER 1}

\section{Literature Review}

\section{Adolescent Obesity in the United States}

Obesity is one of the greatest public health issues facing America today.

Specifically, the growing rate of adolescent obesity is of concern because overweight or obese children are more likely to be obese as adults than normal weight or underweight children (Freedman, Khan, Serdula, Dietz, Srinivasan, \& Berenson, 2005). Currently, more than one third of adults and $17 \%$ of youth in the United States are obese. The prevalence of obesity has more than doubled in children aged 2-5 and quadrupled in adolescents aged 12-19 in the past 30 years (Ogden, Carroll, Kit, \& Flegal, 2014). Healthy People 2020 objective Nutrition and Weight Status (NWS)-10 focuses on reducing the proportion of children and adolescents who are considered obese in the United States. More specifically, objective NWS-10.3 focuses on reducing the proportion of children and adolescents aged 2-19 who are considered obese to $14.5 \%$ by 2020 (HealthyPeople.gov, 2014).

\section{Measures of Obesity}

The terms "overweight" and "obesity" refer to body weight that is greater than what is considered healthy for a certain height (National Institutes of Health, 2012), but measuring and defining overweight and obesity among adolescents by using only one simple index is problematic because their bodies are undergoing a number of physiological changes through the teen years (World Health Organization, 2014). The term "adolescence" is used to refer to those aged 2-19. Measures of overweight and obesity in adolescents are classified using body mass index (BMI) as defined by the 
Centers for Disease Control and Prevention (CDC) standards of BMI assessment.

Whereas adult BMI assessment uses weight and height, adolescent BMI takes into account the sex and age of those aged 2-19 and provides a reliable indicator of body fatness for most children and teens. Both child and teen BMI percentiles are used to track and screen for weight categories that have been associated with increased risk for health problems (Centers for Disease Control and Prevention, 2012a). "Overweight" in children is defined as a BMI at or above the $85^{\text {th }}$ percentile and lower than the $95^{\text {th }}$ percentile for children of the same age and sex. "Obesity" in children is defined as a BMI at or above the 95th percentile for children of the same age and sex (National Institutes of Health, 2012). Table 1.1 shows the weight status categories according to the CDC growth chart percentiles.

Table 1.1: Weight status categories according to the CDC growth chart percentiles (National Institutes of Health, 2012).

\begin{tabular}{|l|l|}
\hline Weight Status Category & Percentile Range \\
\hline Underweight & Less than the $5^{\text {th }}$ percentile \\
\hline Healthy weight & $5^{\text {th }}$ percentile to less than the $85^{\text {th }}$ percentile \\
\hline Overweight & $85^{\text {th }}$ to less than the $95^{\text {th }}$ percentile \\
\hline Obese & Equal to or greater than the $95^{\text {th }}$ percentile \\
\hline
\end{tabular}

\section{Prevalence and Incidence of Adolescent Obesity}

Data from the National Health and Nutrition Examination Survey (NHANES) showed that in 2011-2012, 31.8\% of adolescents were either overweight or obese and $16.9 \%$ were obese. The prevalence of obesity did not significantly differ between boys (16.7\%) and girls (17.2\%) in 2011-2012, but there were significant disparities among different age groups and racial groups. $8.4 \%$ of $2-5$ year olds were obese as compared to $17.7 \%$ of 6-11 year-olds and $20.5 \%$ of $12-19$ year olds (Ogden et al., 2014). 
Although the prevalence of obesity increases with age, incident obesity was highest at the youngest ages and declined through eighth grade (Cunningham, Kramer, \& Venkat Narayan, 2014). Excess body weight early in life is strongly correlated with subsequent obesity risk, and children are becoming obese at very young ages. Data from the Early Childhood Longitudinal Study showed that children at the $50^{\text {th }}$ percentile of BMI at 5 years of age had a $6 \%$ probability of being obese at 14 years of age. This probability increased to $25 \%$ for 5 year-olds at the $85^{\text {th }}$ percentile, $47 \%$ for those at the $95^{\text {th }}$ percentile, and $72 \%$ for those at the $99^{\text {th }}$ percentile. Therefore a component of the course towards obesity is already established by the age of 5 years (Cunningham et al., 2014).

\section{Racial Disparities}

Significant disparities in the prevalence of adolescent obesity exist for racial/ethnic groups in the United States. The prevalence of obesity was significantly lower in non-Hispanic white adolescents (14.1\%) than Hispanic adolescents (22.4\%) and non-Hispanic black adolescents (20.2\%) (Ogden et al., 2012). Data from the Early Childhood Longitudinal Study found a similar pattern for all age groups (Cunningham et al., 2014). Disparities are also seen in incident obesity; $16.8 \%$ of non-Hispanic black adolescents became obese between the ages of 5 and 14 followed by $14.3 \%$ of Hispanic adolescents and $10.1 \%$ of non-Hispanic white adolescents (Cunningham et al., 2014). Figure 1.1 shows the prevalence of adolescent obesity by age, sex, and race. 


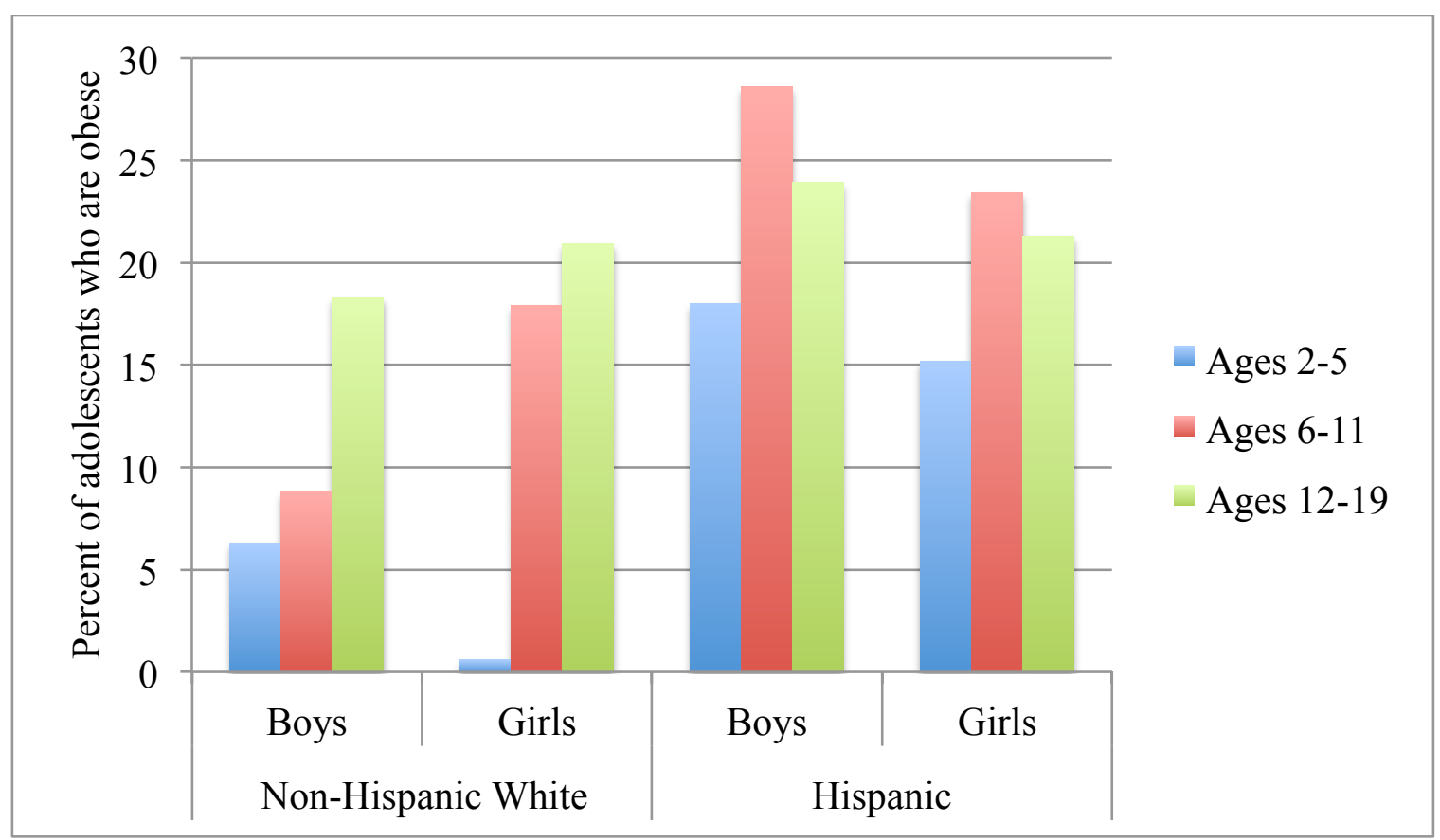

Figure 1.1: Prevalence of adolescent obesity in non-Hispanic white and Hispanic adolescents aged 2-19, by sex and age (Ogden et al., 2012).

Although the reasons behind these disparities are not fully understood, they are likely the result of many interconnected factors. Social and cultural values, low accessibility to fresh foods, economic barriers, and differences in physical activity and food choices may all play a role. A cross-sectional analysis of 1,672 adolescents (mean age $=15$ ) in the Eating and Activity in Teens 2010 found that Hispanic adolescents consumed significantly more fast food than white adolescents (Arcan, Larson, Bauer, Berge, Story, \& Neumark-Sztainer, 2014). Opalinski (2010) determined that busy parent schedules and time constraints may lead to higher fast food consumption and fewer home-cooked meals. Analysis of data from 5801 adolescents aged 12-17 in the 2001 California Health Interview Survey (CHIS) found that significantly fewer Hispanic adolescents met the physical activity recommendations than non-Hispanic adolescents, particularly first-generation (non-US born who has immigrated and become naturalized) and second-generation (US-born with at least one parent non-US born) adolescents 
(Allen, Elliot, Morales, Diamant, Hambarsoomian, \& Schuster, 2007). Third-generation (US-born with at least one grandparent non US-born) Hispanic adolescents demonstrated significantly more time spent watching television and playing video games and lower consumption of vegetables and milk than white adolescents. A generational trend toward worsening dietary habits was observed. First- and second-generation Hispanic youths tended to have dietary habits similar to non-Hispanic whites while third-generation youth tended to have poorer habits. For example, in this sample third-generation Hispanic youths consumed $25 \%$ more soda than whites (Allen et al., 2007). Other studies have suggested that although many ethnic minorities have adopted the Western cultural preference for slenderness, younger generations in pursuit of this may feel constrained by the opinions of older generations' in favoring larger body sizes (Townsend \& Scriven, 2014).

\section{Socioeconomic Disparities}

Disparities in the prevalence of adolescent obesity also exist between socioeconomic (SES) groups. Among both boys and girls obesity prevalence tends to decrease as income increases, but this is not consistent across race/ethnicity groups (Ogden, Lamb, Carroll, \& Flegal, 2010). In one study the prevalence of obesity among $8^{\text {th }}$ graders was consistently highest among those with lower SES status (Cunningham et al., 2014). Data from the Early Childhood Longitudinal Study, which followed a representative cohort of 7738 kindergarteners, found that children from the wealthiest $20 \%$ of families had lower obesity rates than children from all other SES quintiles.

Children in the next-to-poorest quintile had the highest rates of obesity by eighth grade (25.8\%), compared to $24.1 \%$ in the poorest quintile and $11.4 \%$ in the wealthiest quintile 
(Cunningham et al., 2014). Another study demonstrated that those below the poverty threshold were 2.8 times as likely to be obese compared to children (aged 10-17) with family incomes exceeding $400 \%$ of the poverty threshold (Singh, Kogan, Van Dyck, \& Siahpush, 2008). This study (of 46,707 adolescents in the 2003 National Survey of Children's Health) also noted that the obesity prevalence tended to be significantly higher among children from single-parent households and non-English speaking households, from non-metropolitan areas, and from socioeconomically disadvantaged backgrounds (Singh et al., 2008).

The inverse relationship between SES and obesity is complex. In a recent review of the literature, Townsend \& Scriven (2014) noted that those from lower SES background in high-income countries tended to engage in more obesogenic behaviors, such as eating more energy-dense food and being less physically active. This holds true for children, with SES in childhood being a predictor of obesity in adulthood. Additionally, the cost of food may be a significant barrier to adopting healthful dietary behaviors. Studies have shown that energy-dense and nutrient-poor foods provide dietary energy at a lower cost than healthy foods. Links have also been characterized between SES and environmental factors such as likelihood of breastfeeding, nutrition in infancy and childhood, attitudes towards body size and shape, and cultural and social norms in relation to dietary habits, all of which may play a role in the development of obesity (Townsend \& Scriven, 2014). While an environmental approach suggests that food poverty should be addressed with a focus on access, affordability and availability of healthy foods, an individual approach suggests that awareness and acceptability should be 
the focus (Wrieden et al., 2007). Taken together these approaches highlight some of the reasons why food choices in lower SES populations are complicated.

\section{Racial and Socioeconomic Interactions}

The independent influence of race/ethnicity and socioeconomic status is not always clear because individuals from ethnic minorities are more likely to be of low SES and live in poverty in the United States. A study that analyzed data from 46,707 children aged 10-17 in the 2003 National Survey of Children's Health investigated the independent and joint effects of race/ethnicity, poverty status, television viewing, and physical activity as predictors of adolescent obesity (Singh et al., 2008). Results showed that income effects were larger for Hispanics than non-Hispanic whites. The odds of obesity were 2.7 times higher for Hispanic children after controlling for the joint effects model and other social/demographic variables. Factors that were independently associated with a significantly higher risk of adolescent obesity were Hispanic ethnicity, non-metropolitan residence, lower household education, higher poverty levels, low neighborhood social capital, and increased levels of television viewing and physical inactivity. Joint effects by race/ethnicity and SES indicate the potential for considerable reduction in the existing disparities in adolescent obesity in the United States (Singh et al., 2008).

\section{Consequences of Adolescent Obesity}

Adolescence is an extremely important time for the development and persistence of obesity. Adolescence is marked by changes in body composition and decreased insulin sensitivity during puberty; as well as behavioral changes in diet and physical activity; and psychological health (Alberga, Sigal, Goldfield, Prud'homme, \& Kenny, 2012). For 
many reasons addressing healthy body weight is of great importance during, or preferably before, this developmental period. Obese adolescents are more likely to be obese adults (Centers for Disease Control and Prevention, 2014).

\section{Immediate Health Consequences}

Obesity during adolescence presents a number of immediate health effects. Obese adolescents are more likely to have higher blood pressure and higher cholesterol, increasing risk for cardiovascular disease. A cross-sectional analysis of the Bogalusa Heart Study participants (10,099 adolescents aged 5-17) showed that $70 \%$ of obese children had at least one risk factor for cardiovascular disease, and 39\% had two or more (Freedman, Mei, Srinivasan, Berenson, \& Dietz, 2007). Obese adolescents are also more likely to have prediabetes, a condition of high blood glucose levels that indicates an increased risk of type 2 diabetes (Li, Ford, Zhao, \& Mokdad, 2009). Other immediate health effects may include breathing problems such as sleep apnea and asthma, bone and joint problems, fatty liver disease, gallstones, and gastro-esophageal reflux disease (Centers for Disease Control and Prevention, 2014).

\section{Long-Term Health Consequences}

Studies have shown that children who became obese as early as age 2 were more likely to be obese as adults (Freedman et al., 2005). Excess body weight in children is associated with excess morbidity (incidence of disease) in childhood and into adulthood, and increases the risk of a multitude of health problems including heart disease, hypertension, type 2 diabetes, stroke, gallstones, breathing problems, and several types of cancer (Kushi et al., 2006). 


\section{Psychological \& Social Consequences}

Overweight and obese children are more likely to experience stigmatization, teasing, bullying, and ostracizing by their peers, which can lead to social isolation, depression, anxiety, and low self-esteem (Jacobson \& Melnyk, 2011). A recent review determined numerous psychological co-morbidities associated with adolescent obesity, including Attention Deficit Hyperactivity Disorder (ADHD), internalizing/externalizing disorders such as anxiety and depression, and sleep problems (Pulgarón, 2013).

\section{Economic Consequences}

Obesity and its associated health problems present a significant economic impact on the U.S. health care system. This impact includes both direct and indirect costs. Direct costs may include preventative, diagnostic, and treatment services related to obesity while indirect costs may include the value of income lost from decreased productivity, reduced activity (Centers for Disease Control and Prevention, 2012b). Cawley and Meyerhoefer (2012) estimated the health care costs of obesity in the U.S. to be as high as $\$ 190$ billion in 2005, a number that is double earlier estimates. These costs are expected to rise, along with obesity rates, over the coming decades (Cawley \& Meyerhoefer, 2012). The growing rate of adolescent obesity is setting the stage for tremendous health costs in the future.

\section{A Complex of Interrelated Factors Causing Adolescent Obesity}

Obesity is a complex health issue and there are many interwoven factors that play a role in the development of obesity. The World Health Organization states that the fundamental cause of adolescent obesity is an energy imbalance between calories consumed and calories expended. This imbalance is attributable to a number of factors 
including a global shift towards consuming energy dense foods that are high in calories and low in micronutrients and a trend towards decreased physical activity (World Health Organization, 2014). However, adolescents are faced with many barriers to healthy eating. Barriers that may contribute to obesity include biological, environmental, psychological, and behavioral factors. An appropriate model that has traditionally been used to visualize the overlapping causes of adolescent obesity is the Biopsychosocial Model (Figure 1.2), which shows how biological, psychological, and social factors all play interrelated roles (Skelton, DeMattia, Miller, \& Olivier, 2006).

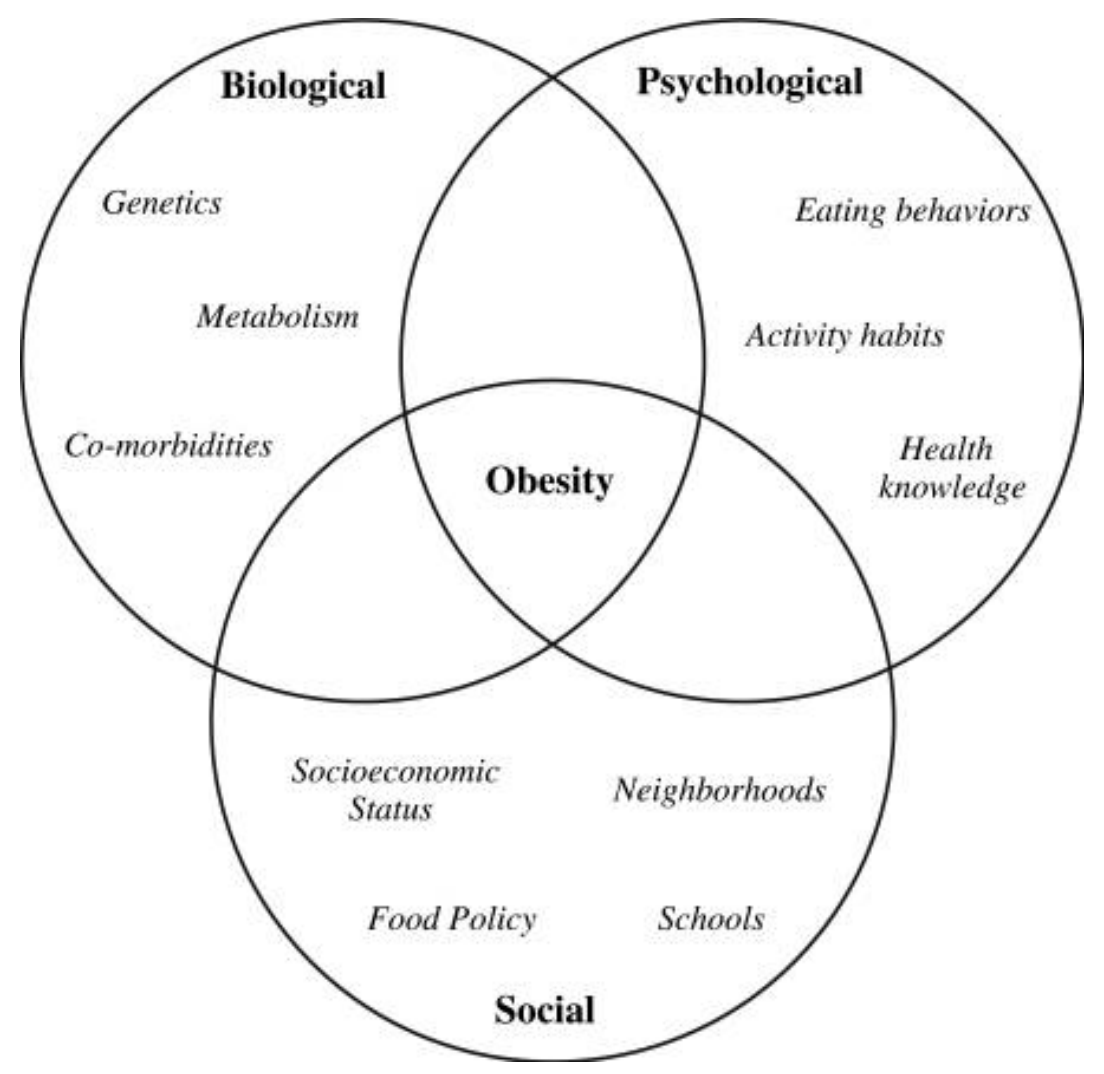

Figure 1.2: Biopsychosocial model of obesity (Skelton et al., 2006).

\section{Biological Factors}

Many genes and genetic markers have been linked with adolescent obesity (Winter, Sankowski, \& Back, 2013). Large-scale association studies in the past decade 
have identified hundreds of specific genes associated with obesity, and the number continues to increase. However, all discovered genetic polymorphisms taken together only account for $2 \%$ of common obesity (Winter et al., 2013). Obesity is a complex condition influenced by the interaction between genetic factors and environmental factors (Butte, Cai, Cole, \& Comuzzie, 2006). Adolescents with a given genetic propensity to becoming obese will be more likely to become so when exposed to an obesogenic environment including readily available food and sedentary behaviors. Genetic predisposition to obesity is expressed when changes in energy intake, energy expenditure, and partitioning of energy to adipose tissue contribute to a positive energy balance (Butte et al., 2006). Therefore

High rates of adolescent obesity in the Hispanic population have warranted genetic investigation. Although the mechanisms of action are still unknown, several processes have been recognized. Butte et al. (2006) designed a Genome-wide Association Study (GWAS) called Viva la Familia to genetically map the phenotypic variation in childhood obesity and it's comorbidities in the Hispanic population. The cohort consisted of 1030 children from 319 families selected based on an overweight proband (a person serving as the starting point for the genetic study of a family) between the ages of 4-19. It was found that sex, age, and environmental covariates explained 1-91\% of the phenotypic variance. Heritabilities of anthropometric indices ranged from $0.24-0.75$, heritability coefficients for the body composition traits ranged from $0.18-0.35$, and diet and physical activity presented heretabilities of $0.32-0.69$. Furthermore, risk factors for metabolic disease were heritable with coefficients of $0.25-0.73$. The Viva la Familia study provides 
strong evidence for a link between genetics and the high prevalence of obesity and its comorbidities in Hispanic children.

\section{Social and Environmental Factors}

Work by Gary Popkin (1993) hypothesizes that a "nutrition transition" in the US is a main causal factor in rising rates of obesity. This transition is marked by a dietary increase in saturated fat, sodium, sugar, and processed/fast foods, often accompanied by a sedentary lifestyle, and the associated high levels of chronic and degenerative diseases (Popkin, 1993). Evolutionarily speaking, our bodies are suited for an environment in which food is scarce and high levels of physical activity are required. The opposite is true in the modern environment which has been termed "obesogenic" due to the high availability of food and low rates of physical activity. The human tendency to gorge and store fat in times of food excess is no longer suitable when there are no lean times to compensate (Townsend \& Scriven, 2014). According to Stevenson, Doherty, Barnett, Muldoon, \& Trew (2007), an obesogenic culture of eating amongst young people has become integrated and normative. These changes have been attributed to an environment that encourages indulgent consumption of energy-rich foods, the promotion of such foods by the media and commercial concerns, and the increasing centrality of these foods in a variety of social contexts.

Townsend \& Scriven (2014) described in a review that urban environments may promote adolescent obesity through the high concentration of fast food chains and convenience stores offering calorically dense foods. People in urban areas tend to be less physically active due to factors such as safety concerns and a lack of proper facilities/areas for exercise (Plantinga \& Bernell, 2007). An increased distance to grocery 
stores is associated with a poorer diet, and poor neighborhoods tend to have more fast food and convenience stores that limit healthy food options (Morland, Wing, \& Diez Roux, 2002). Rural environments may also promote obesity for this reason, as they tend to have fewer supermarkets and limited food choices. Such communities may fall into the category of "food deserts," which are defined as urban neighborhoods and rural towns without ready access to fresh, healthy, and affordable food (USDA Agricultural Marketing Service, 2014). Lack of access often results in poor diet quality which may lead to obesity and other chronic diseases such as heart disease and cancer.

Schools are also important environments that may influence dietary and physical activity behaviors. Variations in school food service results in many schools offering nutritionally imbalanced meals and high amounts of processed and nutritionally imbalanced meals through such channels as vending machines, cafeterias, and food trucks (Townsend \& Scriven, 2014).

\section{Psychological and Behavioral Factors}

\section{Eating Behaviors and Food Choices}

Nearly the entire U.S. population was found to not meet of the current Federal Dietary Recommendations. A 2010 study determined using NHANES data from 16,338 people in the United States found that although people of all ages were not meeting the dietary recommendations, young adults most frequently fell short (Krebs-Smith, Guenther, Subar, Kirkpatrick, \& Dodd, 2010). For example, the proportion of adolescent boys aged 9-13 who did not meet the minimum dietary recommendation was $78 \%$ for total fruit, $96 \%$ for total vegetables, nearly $100 \%$ for dark green vegetables, $98 \%$ for dark green vegetables, $98 \%$ for legumes, $68 \%$ for milk, $99 \%$ for whole grains, $52 \%$ for meat 
and beans, and 75\% for oils. The proportions for adolescent girls aged 9-13 were very similar, with a few notable discrepancies: $84 \%$ did not meet the minimum dietary recommendation for milk and $77 \%$ did not meet the minimum dietary recommendation for meat and beans. Additionally, the proportion of adolescent boys and girls aged 9-13 who exceeded the maximum discretionary energy allowances for solid fats and added sugars was $97 \%$ and $98 \%$, respectively (Krebs-Smith et al., 2010)

As adolescents become more independent, they often begin to make their own food choices. According to Story, Neumark-Sztainer, \& French (2002), diet quality tends to decline from childhood to adolescence due to factors such as eating out more often, concern with physical appearance and body weight, the need for peer acceptance, and busy schedules. Data suggests that the majority of young adults are consuming diets high in fat and low in calcium-rich foods, fruits, deep-yellow and green vegetables, and whole grains (HealthyPeople.gov, 2014). Furthermore, such dietary patterns have been associated with increasing consumption of food prepared away from the home, which tends to be less healthful than foods prepared at home (Larson, Perry, Story, \& NeumarkSztainer, 2006)

A qualitative study examining potential conceptual, physical, individual, developmental, and social barriers to healthy eating was conducted using focus groups with 73 adolescents selected from a range of SES groups and rural/urban locations across Scotland (Stevenson et al., 2007). Four key themes were identified. The first theme identified suggested that the rewarding physical and emotional sensations from eating unhealthy foods leads to an overall preference for sweets and the opinion that healthy foods are tasteless. The second theme found a perception that foods are either "good" or 
"bad," which may cause adolescents to view their diet not as a whole, but broken into specific healthy and unhealthy foods. The third theme identified contradictions that adolescents perceive related to healthy eating. One example of this contradiction theme would be when parents and teachers reward good behavior with unhealthy sweets. The fourth theme suggested that adolescents view healthy eating as a quick fix solution to the obesity endemic rather than a long-term solution and that a healthy diet is something that only needs to be adopted when health has already deteriorated (Stevenson et al., 2007). Understanding these specific barriers is key when exploring ways to prevent adolescent obesity.

\section{The Importance of Culinary Skills for Healthful Eating}

Cooking from scratch is no longer the norm in American kitchens. Dramatic changes in domestic cooking practices have led to a decrease in the transfer of cooking skills from parent to child. It can be argued that Western culture has transformed cooking into a means to an end rather than a process for social and cultural expression. Levy \& Auld (2004) confirm that changes in American demographics, lifestyle, education, and access to convenience food and fast food have led to a reduction in the transfer of cooking skills both in family and educational settings. Cooking and home economics classes are no longer formally taught in most schools. Many believe that this decline represents a sociodemographic shift marked by changes in family structure, women in the workforce, and time constraints (Nelson, Corbin, \& Nickols-Richardson, 2013). Cost and convenience of food are weighted more than ever before as predictors of food choice.

Because people's daily lives tend to be influenced by a chronic feeling of time constraint, they tend to adopt time-saving eating behaviors such as choosing processed/convenience 
foods and fast foods and spending much less time preparing and eating food (Hartmann, Dohle, \& Siegrest, 2013). The widespread decline in cooking skills is strongly linked to poor diet quality and adolescent and adult obesity (Condrasky \& Hegler, 2010). Luckily, in the past decade there has been an interest in reviving culinary skills education for adolescents.

Many studies have investigated the impact of food preparation on diet quality, and overall a positive association is clear. Cross-sectional analyses were performed on data from the Project EAT (Eating Among Teens) survey which included an ethnically and socioeconomically diverse sample of middle school and high school students. An analysis of the entire sample $(\mathrm{n}=4,746)$ by Larson, Story, Eisenberg, \& Neumark-Sztainer (2006) found that adolescent food preparation was related to more healthful food choices. Preparing food was positively associated with fruit and vegetable consumption in female adolescents and fruit consumption in male adolescents $(P<0.01)$. Furthermore, adolescents who reported preparing food seven times in the past week were found to report consumption of at least an additional one-half serving of fruits and one-half serving of vegetables than adolescents who reported never preparing food. Food preparation also presented a negative correlation with soft drink consumption in females and fried food consumption in males $(P<0.01)$.

Larson, Perry et al. (2006) analyzed data from a follow-up survey sent five years after the original Project EAT study (764 males and 946 females). Results confirmed that young adults who reported more frequent food preparation more often met the dietary objectives of Healthy People 2020 and less often consumed fast food. The majority of young adults did not perform food preparation behaviors during a typical week, and the 
most common barrier to food preparation was lack of time (Larson, Perry et al., 2006). Improving skills in the kitchen has been shown to lead to greater self-efficacy to prepare food, improved knowledge of healthy foods, increased frequency of cooking at home, and increased consumption of fruits and vegetables (Condrasky, Williams, Catalano, \& Griffin, 2011). Laska, Larson, Neumark-Sztainer, \& Story (2012) found that helping to prepare food for dinner during adolescence was significantly associated with higher enjoyment of cooking 10 years later among both sexes. This study also suggested that participating in food preparation during emerging adulthood predicts better dietary habits in mid-to-late twenties (Laska et al., 2012). There is no shortage of evidence suggesting that culinary skills may lead to improved diet quality and many feel that teaching adolescents to cook may reduce obesity (Nelson et al., 2013).

A problem in demonstrating how learning culinary skills leads to improved dietary quality and how this might then lead to a decrease in adolescent obesity is a lack of scientific evidence documenting and explaining causal pathways. One reason for this research gap is the lack of a reliable, standardized cooking skills scale. Measurements of cooking skills are subject to cultural and traditional influences as well as personal preferences and eating habits (Nelson et al., 2013).

A behavior such as eating healthfully is unlikely to occur if a person does not have the both knowledge and skill to do so (Glanz, Rimer, \& Viswanath, 2008). For example, federal dietary guidelines simply inform people about healthy food choices to make and good eating practices to follow. However, nutrition principles must be applicable in order to be useful. In order to translate these principles into actual meal 
preparation, they must be implemented using appropriate culinary skills (Hartmann et al., 2013).

\section{The Importance of Social and Family Norms for Healthful Eating}

Among the most important factors when considering adolescent obesity and healthful eating are peer and family influence and perceived social and family norms. According to Glanz et al. (2008), perceived norms refer to the pressure one feels to perform or not perform a particular behavior, which includes beliefs about what others think (injunctive norms) along with beliefs about what others are doing (descriptive norms). Perceived norms are a function of underlying normative beliefs, which are beliefs about the extent to which others think one should or should not perform a particular behavior (Glanz et al., 2008).

\section{Perceived Social and Peer Influence}

Peers serve as influential role models as adolescents spend increasingly more time together, and research has shown that personal interests, values, goals, and commitments are strongly impacted by the beliefs of peers (Nelson et al., 2013). Recently social norms have been linked with adolescent obesity and eating patterns because groups of peers tend to adopt similar behaviors. Adolescent weight gain was correlated with friend and family weight gain in a recent analysis of a social network of 12,067 people as part of the Framingham Heart Study (Christakis \& Fowler, 2007). Ball, Jeffery, Abbott, McNaughton, \& Crawford (2010) determined that social norms for both healthy eating and physical activity significantly predicted these behaviors. Fast food consumption $(P<0.0005)$, soft drink consumption $(P<0.0005)$, and fruit and vegetable consumption $(P<0.005)$ were all significantly associated with social norms regarding the behavior. 
Peers tend to influence one another through observational learning, which raises selfefficacy by increasing the belief that one can complete a task equally well. Salvy, de la Haye, Bowker, \& Hermans (2012) found through a review of empirical literature that adolescents were strongly influenced by nutrition related behaviors of peers in their social network, and that tasting healthy foods becomes the norm when adolescents observe peers trying them. Furthermore, a review by Turner (1999) confirmed that youths are more likely to sustain healthy behaviors if they feel empowered by their peers to do so.

Few studies have explored the association between social norms and culinary habits in adolescents (Nelson et al., 2013). Liquori, Koch, Ruth Contento, \& Castle (1998) suggested that preparing and eating foods in a positive social context may enhance food preferences and familiarity with healthy foods. Lukas \& Cunningham-Sabo (2011) identified through focus groups with adolescents who participated in the Cooking With Kids program $(\mathrm{n}=178)$ that school-aged children likely enjoy sharing experiences in the kitchen with peers and that it can increase acceptance of, and preference for, healthy foods. Focus groups with teachers $(\mathrm{n}=17)$ of these same students felt that culinary programs allowed adolescents to practice team-building skills and encouraged them to treat peers with respect (Lukas \& Cunningham-Sabo, 2011). The potential for increasing healthy eating habits in adolescents through the modification of social norms is promising and warrants further research.

\section{Perceived Family Norms and Influence}

Family also has a significant influence on adolescents' eating habits and risk of becoming obese. As mentioned previously, children have a higher likelihood of becoming overweight or obese if at least one of their parents is (Christakis \& Fowler, 
2007). This is likely due to a combination of biological, social, environmental, and behavioral factors. Previous studies have shown that adolescents' perceptions of parent support for healthy eating were associated with better diet quality, such as higher fruit and vegetable intake and less snacking (Fitzgerald, Heary, Kelly, Nixon, \& Shelvin, 2013). The presence of a parent at mealtime is associated with higher intake of fruits, vegetables, dairy food, and breakfast, and consuming a high frequency of family meals has been associated with a healthier diet overall (Townsend \& Scriven, 2014).

Furthermore, fruit and vegetable intake in adolescents can be strongly predicted by parental fruit and vegetable intake since parents serve as both role models and decision makers for food choices and preferences. Parents shape their children's dietary patterns by setting examples of meal and snack patterns, consumption of food away from the home, beverage consumption, portion sizes, and overall diet quality (Nicklas, Baranowski, \& Baranowski, 2001).

\section{Approaches to Prevent and Treat Adolescent Obesity}

Although there are many approaches to treating adolescent obesity, research has indicated that educating adolescents about making diet and lifestyle changes is extremely promising for eliciting long-term change. Health promoting interventions implemented earlier in life that target modifiable risk factors such as diet and lifestyle are likely to have a positive effect on the prevention of child and adult onset obesity (Branscum \& Sharma, 2012). Zeinstra, Koelen, Kok, \& de Graaf (2007) further confirm this idea, stating that interventions focused on young children impact behaviors before they are established, which may lead to more permanent effects. Furthermore, children aged 7-12 have the cognitive capacity to understand the health benefits of foods and can identify specific 
taste differences (Wall, Least, Gromis, \& Lohse, 2012). A review that assessed the efficacy of lifestyle, drug, and surgical interventions for treating obesity in childhood found that combined behavioral lifestyle interventions compared to standard care or selfhelp can produce a significant and clinically meaningful reduction in overweight and obese children and adolescents. Although drug therapies produced significant results when given in addition to a lifestyle program in moderate to severely obese adolescents, a range of adverse effects were noted (Oude Luttikhuis et al., 2009).

Nutrition and culinary education with a focus on increasing knowledge and skills related to food choices have shown positive outcomes. Significant disparities in the prevalence of adolescent obesity according to race/ethnicity and socioeconomic status indicate a need for culturally and socially relevant interventions. According to Condrasky \& Hegler (2010), "culinary nutrition" is a necessary practicality to improve the health of our nation. Culinary nutrition is defined as the application of nutrition principles with food science knowledge displayed through a mastery of culinary skills, which results in healthy eating behaviors produced from culinary confidence and nutrition knowledge. The differing worlds of culinary arts and nutrition science must be merged in order to successfully translate nutrition concepts and healthy cooking techniques into sustainable eating practices for the future health of adolescents in the US (Condrasky \& Hegler, 2010).

To develop effective nutrition and culinary education programs for adolescents it is necessary to understand the factors that determine eating behavior in this population. Research has continually shown that theory-based interventions that are guided by behavioral change theories are more likely to have a significant impact on dietary 
behaviors in adolescents (McClain, Chappuis, Nguyen-Rodriguez, Yaroch, \& SpruijtMetz, 2009). These theories are fundamental to understanding health behaviors because they provide a framework by which to examine the relationships among constructs.

\section{Integrated Behavioral Model for Nutrition and Culinary Education}

The Integrated Behavioral Model (IBM) includes constructs from many preexisting and widely used models such as the Theory of Reasoned Action, Theory of Planned Behavior, Health Belief Model, and Social Cognitive Theory. (See Figure 1.3.) In the IBM the most proximal determinant of a behavior is the intention to perform the behavior, and motivation is essential to intention. Four other components directly affect behavior, making it more likely to occur: knowledge and skills to carry out the behavior; no or few environmental constraints that limit behavioral performance; salience (importance) of the behavior; and habitual tendency toward the behavior. These four components and their interactions are important to consider when designing healthpromoting interventions, as they indicate strong behavioral intention. Behavioral intention is determined by three constructs: attitude, perceived norm, and personal agency. These constructs are all functions of underlying beliefs (Glanz et al., 2008).

The IMB may be used as an effective tool for guiding nutrition and culinary interventions that target specific behaviors such as consuming and preparing healthy foods in adolescents. It is important to understand each of the constructs and how they influence behavioral determinants and intentions. 


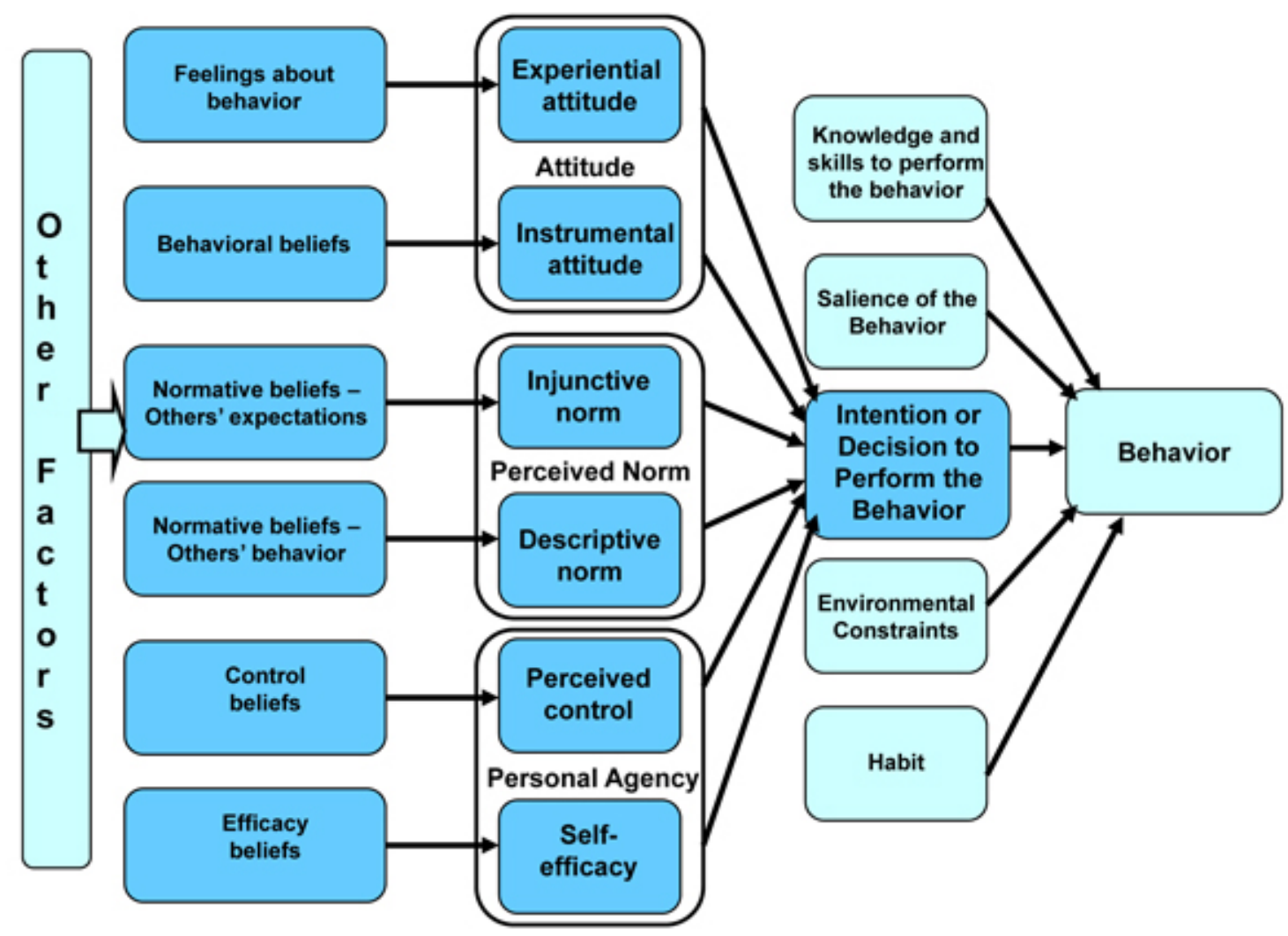

Figure 1.3. The Integrated Behavioral Model (Glanz et al., 2008).

\section{Constructs}

\section{Attitude}

Attitude toward the behavior, as defined by Glanz et al. (2008), is a person's overall acceptance or opposition toward performing the behavior, which is composed of affective and cognitive dimensions. Experiential attitude, or affect, refers to the emotional (positive or negative) response of the individual towards performing the behavior. A negative response predicts that the behavior is unlikely to be performed; a positive response predicts that the behavior is likely to be performed. Instrumental attitude, which is cognitively based, determines a person's beliefs about the outcomes behavioral performance (Glanz et al., 2008). Instrumental attitude corresponds to the SCT concept of outcome expectancies, defined as beliefs about the likelihood of various outcomes of a 
specific behavior. Very few studies have evaluated the link between outcome expectancies and dietary quality, and results have not been consistent (McClain et al., 2009). However, positive associations have been found between positive outcome expectancies and fruit and vegetable consumption (Resnicow et al., 1997; Keihner, Meigs, Sugerman, Backman, Garbolino, \& Mitchell, 2011). Experiential and instrumental attitudes are a function of underlying beliefs and feelings about the outcomes of performing the behavior. For example, positive underlying feelings and beliefs about preparing healthy food will likely lead to a more favorable attitude about preparing healthy food. Establishing positive emotional responses and outcome expectancies towards cooking and healthful eating is crucial in nutrition and culinary education.

\section{Perceived Norms}

Perceived norms reflect the social pressure one feels to perform or not perform a particular behavior (Glanz et al., 2008). Injunctive norms (beliefs about what others think and believe) along with descriptive norms (beliefs about what others are doing) make up this construct, which emphasizes the strong influence of social identity in many cultures. Perceived norms are a function of underlying normative beliefs (also called subjective norms), which are an individuals beliefs about the extent to which other people who are important to them think they should or should not perform a behavior. For example, the stronger one's beliefs that others think one should consume healthy food or that others are consuming healthy food, the stronger is one's perception of social pressure to consume healthy food (Glanz et al., 2008). As described earlier, perceived social and family norms are an important determinant of eating behaviors in adolescents. Nutrition and culinary education may have the potential to influence perceived social norms 
through group work, peer interactions, and observational learning. Perceived family norms may be influenced through including family members in the intervention or providing ways for the participants to extend their knowledge and skills into the home environment.

\section{Personal Agency}

Personal agency refers to individual's capability to originate and direct actions for given purposes (Glanz et al., 2008). This construct is made up of perceived control (an individual's perceived amount of control over behavioral performance) and self-efficacy (an individual's confidence in his/her ability to perform specific tasks in the face of obstacles and challenges). Personal agency is a function of underlying beliefs about control and efficacy for a given behavior. For example, the stronger one's beliefs that they have the ability to prepare healthy foods despite various barriers, the greater one's self-efficacy to prepare healthy foods will be (Glanz et al., 2008).

\section{Self-Efficacy}

Self-efficacy is often regarded as one of the most important factors in predicting behavioral performance. Fitzgerald et al. (2013) defines dietary self-efficacy as the perceived capability to make healthy food choices, even when faced with potential barriers. Research has shown that higher dietary self-efficacy is related to healthier food choices, such as higher fruit and vegetable intake. A 2013 study of 483 adolescents aged 13-18 found that self-efficacy for eating and peer support for unhealthy eating are both associated with food intake patterns. For example, higher self-efficacy beliefs were related to healthy food intake, and lower self-efficacy beliefs were related to unhealthy food intake (Fitzgerald et al., 2013). 
The relative importance of each of the three theoretical constructs may vary for different behaviors and different populations. For example, intention to perform a behavior such as cooking a meal from scratch may largely depend upon personal agency and self-efficacy for cooking (Glanz et al., 2008). Thus, to design effective interventions to influence behavioral intentions it is important to determine the degree to which each construct influences the intention to perform the behavior.

\section{Obesity Prevention through Nutrition and Culinary Education}

The IBM suggests that in order for adolescents to adopt a behavior such as healthful eating and food preparation, intention and motivation must be present. In order for intention and motivation to be present, they must possess the knowledge and skills to perform the behavior, there must be no or few environmental constraints, and the behavior must be salient and habitual. Furthermore, they must possess a positive attitude about the behavior, they must feel that the behavior is compatible with social and family norms, and they must perceive control and self-efficacy regarding the behavior.

Therefore, a successful nutrition and culinary education program designed to inspire healthful eating and food preparation in adolescents should aim to improve knowledge and skills, attitude, perceived norms, and self-efficacy related to the behavior in a safe, encouraging environment free of constraints. Each of the following examples of nutrition and culinary interventions address at least one specific construct or behavioral determinant of the IBM.

\section{Chef and Child Foundation Program}

Dougherty \& Silver (2007) evaluated a programed called the American Culinary Federation Chef and Child Foundation Program (C\&C). According to the children's' 
program evaluations $(n=36), 90 \%$ of the participants reported a high level of enjoyment. Chef-nutritionist teams contributed to the park of enjoyment, and as a result the children also gave high marks on future interest in what they learned. This positive class experience combined with practical skill-building exercises increases the likelihood that participants will perform healthy behaviors at home (Dougherty \& Silver, 2007).

\section{Chefs Adopt a School}

A UK-based nutrition and culinary program called Chefs Adopt a School (CAAS) showed positive outcomes related to eating healthfully (Halford, Caraher, Seeley, Wu, \& Lloyd, 2013). Professional chefs worked with local schools to deliver three class sessions to 86 students aged 9-11. Class topics included healthy eating, flavors and taste, practical food preparation, hygiene and health, and the session concluded with a visit to a restaurant. Pre- and post-intervention questionnaires were designed to measure attitudes about the program, changes in individual skills, confidence for cooking, vegetable consumption, and confidence for asking their parents to purchase specific foods. Results demonstrated that, compared to a control group, the program significantly improved confidence for cooking $(P<0.001)$ and significantly increased vegetable consumption $(P=0.002)$ (Halford, et al., 2013).

\section{Cookshop}

Another example of a school-based program linking nutrition education with culinary skills is the Cookshop Program in New York (Liquori et al., 1998). Designed for elementary school students, the goal of the program was to increase preference for, and consumption of, minimally processed whole grains and vegetables through repeated exposure in the cafeteria and hands-on culinary exposures in the classroom. The outcome 
evaluation compared four curriculums: Cookshops plus food and environmental lessons, Cookshops only, food and environmental lessons only, and a control group. Pre- and post-intervention questionnaires were completed by 590 students. Individuals that received Cookshops demonstrated significantly higher mean preference scores for plant foods ( $P<0.001$ for both age groups), knowledge scores ( $P<0.05$ for grades K-3; $P<0.001$ for grades 4-6), and less plate waste $(P<0.01)$ than classes that did not receive Cookshops in both younger and older children. Cookshops also positively impacted behavioral intentions in younger children $(P<0.01)$ and cooking self-efficacy in older children $(P<0.05)$. Food and environmental lessons had a positive impact only on knowledge scores. These results suggest that cooking and eating healthy foods along with peers, accompanied by a learning component, may be an effective nutrition education model for impacting mediators of healthy eating (Liquori et al., 1998).

\section{CookWell}

Wrieden et al. (2007) evaluated a food skills intervention called CookWell aimed at improving cooking confidence, food preparation methods, and dietary choices in reduced income areas of urban Scotland. The 7-week program was delivered to 113 adults and covered topics such as food hygiene, nutrition, interactive question-answer sessions, and guided recipe creation. A pre- and post-intervention questionnaire, food diaries, and a food frequency questionnaire were collected at baseline, completion, and 6 months afterwards in the intervention group and a control group. Immediately following the program, those in the intervention group significantly increased their fruit consumption $(P<0.05)$. At the 6-month follow up, those in the intervention group showed a significant increase in their confidence in following a recipe $(P<0.05)$ (Wrieden et al., 
2007). Despite the practical difficulties of working with low-income populations, such as high attrition and difficulties with the participants' commitment levels, these results suggest that a food skills intervention may have a positive effect on the dietary habits of low SES adults.

\section{Oklahoma Cooperative Extension Service}

A 2005 study found positive outcomes related to fruit and vegetable intake and food safety behaviors in youth and adults $(\mathrm{n}=602)$ following a series of cooking classes developed by the Oklahoma Cooperative Extension Service (Brown \& Hermann, 2005). Previously validated pre- and post-education questionnaires were used to evaluate the effectiveness of the cooking classes. The average number of fruit servings per day increased from 1.1 to 2.3 servings in youth $(P<0.0001)$, and there was a $39 \%$ increase in the number of youth who consumed the recommended 2 fruit servings per day. The average number of vegetable servings per day increased from 1.4 to 2.4 servings in youth $(P<0.0001)$, and there was a $25 \%$ increase in the number of youth who consumed the recommended 3 vegetable servings per day. Furthermore, $36 \%$ of youth increased cooking skills such as using a clean knife and cutting board to prepare fruits and vegetables to avoid cross-contamination $(P<0.0001)$. The degree of benefit was much higher in youths than adults for each variable measured, further confirming the belief that adolescence is a crucial time to establish healthful dietary behaviors (Brown \& Hermann, 2005).

\section{Cooking With a Chef}

School-based culinary programs are on the rise as they continue to produce positive outcomes in adolescents. A 2008 study evaluated a nutrition and culinary 
intervention called Cooking With a Chef (Condrasky, Parisi, Wall-Bassett, \& Warmin, 2008). A chef and a nutrition expert worked together to provide a hands-on five-session series of nutrition topics, cooking tips, and demonstrations. Participants were able to immediately apply information on ways to improve nutritional quality of meals through various culinary techniques. A pre-post intervention survey and focus groups performed after the series identified an increase in cooking skills, confidence level, number of meals prepared at home, discussion and preparation of healthier choices, and the frequency of cooking behaviors and cooking self-efficacy (Condrasky et al., 2008).

\section{LA Sprouts}

Evidence has demonstrated that gardening and nutrition interventions improve dietary intake in children and a 2012 Los Angeles-based pilot study evaluated the effect of this type of intervention on obesity outcomes (Davis, Ventura, Cook, Gyllenhammer, $\&$ Gatto, 2011). Latino adolescents were the focus of this study, given the fact that Latinos in LA are among those with the highest prevalence of obesity and therefore present the highest risk for associated chronic diseases. Furthermore, many Latinos in LA are of low socioeconomic status and reside in food deserts, which poses geographical and financial challenges to maintaining a healthful and balanced diet. A 12-week afterschool gardening, nutrition, and cooking program called LA Sprouts targeted dietary intake and obesity risk in Latino fourth- and fifth-graders. The intervention was delivered weekly for 90-minute sessions over 12 weeks. Results showed that participants $(n=34)$ had significantly increased dietary fiber intake $(P<0.04)$ and significantly decreased diastolic blood pressure $(\mathrm{P}<0.04)$ compared to a control group $(\mathrm{n}=70)$. In a subsample of 18 overweight/obese participants, there was a significant reduction in BMI $(P<0.04)$ and 
significantly less weight gain $(P<0.03)$ compared to a control group of 43 overweight and obese participants. Growing food in homes, schools, or community gardens is a means by which these low-income families increased access to nutritional foods that may otherwise be unavailable. The success of the LA Sprouts program suggests that hands-on, culturally relevant nutrition interventions incorporating a gardening and cooking component can lead to dietary improvements and positive health outcomes in Latino children, including those who are already overweight or obese (Davis et al., 2011).

\section{Cooking With Kids}

A promising 2014 study explored the impact of a school-based nutrition and cooking curriculum on attitudes and behaviors related to preparing and eating fruits and vegetables (Cunningham-Sabo \& Lohse, 2014). The Cooking With Kids curriculum was developed predominantly for low-income Hispanic communities to engage elementary school students in hands-on learning with fresh, affordable foods based on cultural traditions. This study compared the effects of the full Cooking With Kids cooking and tasting curriculum with a less intense, tasting-only curriculum on fourth graders' selfefficacy for cooking, cooking attitudes, and fruit and vegetable preferences. A nontreatment comparison group was also analyzed. Participants were 961 students from eleven low-income public schools in a Southwestern city; $50 \%$ were female and $84 \%$ were Hispanic. The intervention consisted of 5 2-hour cooking and/or 51 -hour fruit and vegetable tasting lessons. A survey was administered before and after the intervention to address each of self-efficacy for cooking, cooking attitudes, and fruit and vegetable preferences. Results from the pre-survey showed that over $80 \%$ of the participants reported cooking experience; $62 \%$ reported making food with friends and nearly $90 \%$ 
reported making food with family. Post-survey results showed that all groups increased self-efficacy over the course of the study, with the greatest impact seen in male students who did not report prior cooking experience $(P=0.004)$. The cooking and tasting curriculum showed a greater increase in self-efficacy as compared to the tasting-only curriculum. Similar to what was seen with self-efficacy the greatest improvement in cooking attitudes was seen in male students without prior cooking experience $(P=0.003)$. For fruit and vegetable preference, the greatest improvement was seen in the cooking and tasting curriculum with male students showing a significantly higher score than females ( $P=0.033)$ (Cunningham-Sabo \& Lohse, 2014).

Lukas \& Cunningham-Sabo (2011) conducted focus group interviews with students and adults prior to the Cooking With Kids program to better understand the students' perceptions of the program and cooking experiences at school and at home. Qualitative results from 22 focus groups ( $\mathrm{n}=178$ fourth-graders) indicated that program participants enjoyed the program and developed more positive attitudes towards cooking. Program participants were also more likely to consider classmates to be close friends than students in the control group, which may indicate that strong social ties were developed during the program. Healthy behaviors are more likely to be sustained if adolescents feel empowered by their peers to do so (Turner, 1999). This study provides evidence that nutrition and culinary interventions conducted in predominantly Hispanic communities may improve attitude and perceived norm, two important constructs that may predict behavior change.

These programs confirm that nutrition and culinary interventions have the potential to improve numerous mediators of healthful eating, and that target populations 
such as Hispanics and students without cooking experience may reap the greatest benefits.

\section{Conclusions}

The adolescent obesity endemic must be targeted through a multifactorial approach with special considerations for people of varying backgrounds, socioeconomic status, race/ethnicity, and family structure (Allen et al., 2007; Singh et al., 2008; Townsend \& Scriven, 2014). Educational interventions are most effective when both knowledge and skills are developed, as seen with nutrition and culinary programs (Condrasky \& Hegler, 2010; Glanz et al., 2008; Hartmann et al, 2013). Adolescence is a key period in which to intervene in order to establish long-term healthy habits (Story et al., 2002; Wall et al., 2012; Zeinstra et al., 2007). Nutrition and culinary programs have demonstrated great potential to improve mediators of healthful eating, such as cooking skills, self-efficacy for cooking, fruit and vegetable preferences, fruit and vegetable consumption, and attitudes towards healthy eating (Condrasky et al., 2008; Liquori et al., 1998; Lukas \& Cunningham-Sabo, 2011). Additionally, such programs may improve obesity-related risk factors such as blood pressure and BMI (Davis et al., 2011). This is an important step in achieving the Healthy People 2020 objective to reduce the proportion of children and adolescents aged 2-19 who are considered obese. Additional research is needed to explore the impact of nutrition and culinary interventions on outcome expectancies and perceived social and family norms in adolescents (McClain et al, 2009; Nelson et al., 2013).

Pink and Dude Chefs, Phase 2, a nutrition and culinary intervention designed to target middle school students in a predominantly Hispanic and low SES community, 
aimed to improve mediators of healthful eating by using the IBM as a framework for behavior change. The unique program under investigation incorporated a multicultural theme into each lesson to enhance knowledge and skill building. Key aspects of the program were peer group work in the kitchen and family-oriented take-home activities. Pink and Dude Chefs, Phase 2 allowed participants to apply nutrition guidelines through the preparation of nutritious meals in a fun, safe environment. The overall goal of the program was to empower youth by providing them with the necessary skills to prepare and consume healthy foods for future benefit to their health and well-being. 


\section{Hypotheses}

Research question \#1: Does participation in the Pink and Dude Chefs, Phase 2 program influence self-efficacy for cooking?

- Research hypothesis \#1: Participation in Pink and Dude Chefs, Phase 2 does influence self-efficacy for cooking.

Research question \#2: Does participation in the Pink and Dude Chefs, Phase 2 program increase cooking skills?

- Research hypothesis \#2: Participation in Pink and Dude Chefs, Phase 2 does increase cooking skills.

Research question \#3: Does participation in the Pink and Dude Chefs, Phase 2 program increase nutrition knowledge related to the USDA MyPlate?

- Research hypothesis \#3: Participation in Pink and Dude Chefs, Phase 2 does increase nutrition knowledge related to the USDA MyPlate.

Research question \#4: Does participation in the Pink and Dude Chefs, Phase 2 program influence outcome expectancies related to eating fruits and vegetables?

- Research hypothesis \#4: Participation in Pink and Dude Chefs, Phase 2 does influence outcome expectancies related to eating fruits and vegetables.

Research question \#5: Does participation in the Pink and Dude Chefs, Phase 2 program influence perceived social and family norms related to cooking?

- Research hypothesis \#5: Participation in Pink and Dude Chefs, Phase 2 does influence perceived social and family norms related to cooking. 
Research question \#6: Are perceived social norms or perceived family norms more highly associated with the eating/culinary behaviors of participants of Pink and Dude Chefs, Phase 2?

- Research hypothesis \#6: Either perceived social or perceived family norms are more highly associated with the eating/culinary behaviors of participants. 


\section{CHAPTER 2}

\section{Materials and Methods}

Pink and Dude Chefs, Phase 2 aims to empower youth by teaching them the necessary practical skills needed to take control over their food choices. Goals of the current iteration of the program were to increase food-related knowledge and skills and to identify factors that may moderate the impact of this curriculum on behavior.

\section{Program Background}

The Pink and Dude Chefs program is made possible by STRIDE (The Center for Solutions Through Research in Diet and Exercise) at California Polytechnic State University. The mission of STRIDE, founded in 2007, is to advance knowledge and practice in obesity prevention by conducting cutting edge, interdisciplinary research, fostering collaborative partnerships among faculty, students and community, and providing real-life learning experiences to develop the next generation of leaders. Pink and Dude Chefs participates in this mission by providing a research-based obesity prevention intervention in afterschool programs in the community.

Pink and Dude Chefs was developed in 2008 by a California Polytechnic State University Nutrition graduate student (Chessen, 2008). Based on the Social Cognitive Theory (SCT), the goals of the program were to increase the participants' self-efficacy for cooking, to build knowledge for healthy dietary practices, and to examine barriers to healthy eating in order to combat adolescent obesity. Designed for use at a middle school as part of an after-school program, early nutrition intervention works to establish healthful eating habits which may persist into adulthood. Results from the 12-lesson (twice weekly for 6 weeks) pilot study showed that program participants demonstrated a 
significant increase in self-efficacy for using basic culinary skills as a result of participation $(P<0.005)$. Chessen's pilot study demonstrated that increasing knowledge and skills in a practical setting is an important step for increasing self-efficacy for choosing nutritious foods.

A reworked curriculum for Pink and Dude Chefs was further investigated by another Nutrition graduate student (Sheehan, 2013). Sheehan focused the curriculum on several factors related to obesity prevention such as fruit and vegetable preferences; dietary behaviors; barriers to healthy eating; culinary skill and culinary confidence, and basic nutrition knowledge. Results of Sheehan's investigation (also twice weekly for 6 weeks) showed that Pink and Dude Chefs increased participants' fruit and vegetable preferences and intake, with a statistically significant increase in overall fruit preference ( $P=0.01)$. Sheehan concluded that increasing the availability and access to health-related cooking education programs such as Pink and Dude Chefs would likely translate into long-term public health benefits.

\section{Phase 2 Curriculum- "Around the World"}

Since its development, the Pink and Dude Chefs program has evolved into two distinct curriculums called "Phase 1" and "Phase 2." Phase 1, utilized by Chessen (2008) and Sheehan (2013), is entitled "Let's Get Started" and focuses on introductory nutrition concepts and culinary skills. A preliminary Phase 2 curriculum titled "Around the World" was created in 2010, but it was not fully developed or systematically examined. This project finalized the Phase 2 curriculum in order to determine its efficacy of Phase 2 as an effective curriculum of Pink and Dude Chefs. The "Around the World" theme is designed to teach participants key nutrition and culinary concepts through a multicultural 
approach, while still maintaining the core obesity prevention framework of Pink and Dude Chefs.

The "Around the World" curriculum introduces participants to a new country each lesson, including France, Italy, Japan, Greece, Thailand, and Morocco. Students are taught general information about each country such as geography and population, followed by interesting facts, national health statistics, and information about the food culture such as customs, etiquette, and meal patterns. The curriculum is designed for students to use critical thinking to make connections between each country's food culture and health status and to draw conclusions about how those patterns compare to the United States food culture and health status. These relationships are reinforced through cooking recipes specific to each country and learning new cooking skills.

\section{Program Support}

The majority of kitchen equipment used in Pink and Dude Chefs, Phase 2 (i.e. knives, cutting boards, mixing bowls, chef hats \& aprons, and food processors) was purchased through a grant from the Maxwell Foundation for the pilot study (Chessen, 2008). The Food Bank Coalition of San Luis Obispo County donated weekly food ingredients needed for recipe creation, and supplementary items were purchased at Food 4 Less through the grant funding from the Maxwell Foundation. Additional funding for supplies and materials came from Cal Poly IRAs (Instructionally Related Activities).

\section{Curriculum Design}

The current research project examining the Pink and Dude Chefs, Phase 2 curriculum took place during two subsequent 10-week academic quarters: Fall 2013 and Winter 2014. During each of these quarters, the session consisted of a two-hour lesson 
each week for eight weeks total. Each two-hour lesson consisted of a classroom portion and a kitchen portion. Weekly topics and recipes were related to the overall theme of Phase 2, "Around the World." A specific country was used as the focus of each lesson; topics included the food culture and customs, meal patterns, health status, and recipes of the country. Each of the two eight-week sessions was intentionally run essentially the same during each quarter therefore data from both quarters were combined for analyses of hypotheses.

\section{Staffing}

The STRIDE Health Ambassadors Program Coordinator developed the Around the World curriculum and authored this thesis in partial fulfillment of a Master's degree. The Program Coordinator also implemented the weekly lessons which included lesson plan preparation, gathering of materials needed weekly, managing additional research assistance staff, and leading the classroom and kitchen lessons. Additionally, the Program Coordinator was responsible for all data collection and handling.

The Program Coordinator was assisted by several graduate and undergraduate Cal Poly students as research assistance staff during each session. Weekly research assistants included two STRIDE Site Managers who were responsible for assisting the Program Coordinator, grocery shopping for recipe ingredients, and helping to lead each lesson. Additional research assistants consisted of Cal Poly students enrolled in the KINE 290-01 Health Ambassadors course, of which there were typically fifteen students per academic quarter. Students enrolled in this course (called "Health Ambassadors") were required to complete the National Institutes of Health (NIH) Protecting Human Subjects Research online program. Health Ambassadors were trained in class to assist in teaching the 
weekly lessons for Pink and Dude Chefs. Health Ambassadors then signed up to participate in least two Pink and Dude Chefs lessons throughout the quarter. The Program Coordinator ensured that three to four Health Ambassadors would be attending each lesson. This level of participation ensured for quality control since it takes at least six people to run a Pink and Dude Chefs lesson per week. The Program Coordinator, two Site Managers, and four Health Ambassador volunteers staffed a typical lesson; resulting in a typical ratio of six to eight staff members to eight to ten students. The high ratio of staff to students is most likely not typical or absolutely necessary to run the program, as it is possible to do so with fewer staff members.

\section{Weekly Lessons: Classroom Portion}

Each lesson began with a forty-five minute classroom portion. Tasks included taking roll, collecting returned goal sheets (explained in further detail in the following section), discussing the recipe prepared the previous week, and introducing the current topic country through a slideshow presentation. The presentations were interactive and encouraged classroom discussion. For example, the Program Coordinator asked questions throughout each presentation that sparked conversation related to the topic country. In addition to learning about the country's food culture and cuisine, nutrition topics such as the USDA MyPlate were introduced. USDA MyPlate was used to teach nutrition knowledge concepts such as food groups, portion sizes, and guidelines from the " 10 Tips Nutrition Education Series.” For example, participants were asked to match staple foods from each country with the correct food group using a MyPlate graphic. As designed, no topic country was explored during the introductory lesson; instead most of the lesson time was spent on the pre-survey and orientation activities. During lessons 2-7 the 
countries explored were France, Italy, Japan, Greece, Thailand, and Morocco. After the slideshow presentation, the recipe of the day was reviewed in detail. Teaching each recipe of the day emphasized any new ingredients being used, specific measurements, and/or any new cooking techniques to be used. Food safety standards were taught and modeled as part of each lesson. The participants were required to wash their hands and put on chef hats and aprons before heading into the kitchen.

\section{Goal Sheets to Extend Learning Outside the Classroom}

In an effort to extend participants' learning and practice of cooking skills outside of the Pink and Dude Chefs setting, 'goal sheets' were developed as a take-home assignment. These one-page handouts asked the participant to complete one of two listed activities, or goals, related to lesson content. The participant was asked to have a parent or guardian sign the goal sheet and bring it back on the next lesson. The incentive for returning a completed goal sheet was earning a raffle ticket for the Family Fiesta celebration (explained in further detail on page 45). Table 2.1 includes the weekly goals for each lesson of Pink and Dude Chefs, Phase 2 aligned with the weekly topic country.

Goals sheets were designed to include one activity related to the participant's family (e.g. "By next week I will teach someone in my family how to properly use a chef's knife") and one activity related to the participant's peers (e.g. "By next week I will ask three of my friends if they have ever tried quinoa"). The intent of providing an opportunity for participants to choose between family- or peer-related activities was to determine associations between perceived social and family norms and nutrition and cooking behaviors. Therefore in data analysis an evaluation of selecting either a familybased or friend based goal could be done to examine whether family or peers had a 
greater influence on participant's cooking attitudes and eating behaviors. [See Appendix B for weekly goal sheets.]

Table 2.1: Pink and Dude Chefs, Phase 2: Goals for the lesson.

\begin{tabular}{|c|c|c|}
\hline Lesson & Topic & Goals \\
\hline Lesson 1 & $\begin{array}{l}\text { Introduction / } \\
\text { California } \\
\text { Cuisine }\end{array}$ & $\begin{array}{l}\text { - I will teach someone in my family how to properly use a chef's } \\
\text { knife when chopping vegetables. } \\
\text { - I will ask one or more friends if they have ever tried quinoa. }\end{array}$ \\
\hline Lesson 2 & France & $\begin{array}{l}\text { - I will use fruit as a sweet, healthy dessert alternative at a family } \\
\text { dinner. } \\
\text { - I will walk or bike with a friend to a destination instead of } \\
\text { driving. }\end{array}$ \\
\hline Lesson 3 & Italy & $\begin{array}{l}\text { - I will use olive oil instead of butter in a recipe or meal with my } \\
\text { family. } \\
\text { - I will explain why the Mediterranean Diet is healthy to one of } \\
\text { my friends. }\end{array}$ \\
\hline Lesson 4 & Japan & $\begin{array}{l}\text { - I will try choosing (or preparing) seafood or tofu instead of } \\
\text { meat during a family mealtime. } \\
\text { - I will teach one of my friends how to use chopsticks. }\end{array}$ \\
\hline Lesson 5 & Greece & $\begin{array}{l}\text { - I will try making pesto or another yummy recipe in a food } \\
\text { processor or blender with my family. } \\
\text { - I will tell } 3 \text { of my friends how to make easy, delicious pizza } \\
\text { using a whole-wheat pita. }\end{array}$ \\
\hline Lesson 6 & Thailand & $\begin{array}{l}\text { - I will use fresh herbs such as cilantro, basil, or mint to garnish } \\
\text { a meal with my family. } \\
\text { - I will ask } 3 \text { friends if they have ever tried Thai food. }\end{array}$ \\
\hline Lesson 7 & Morocco & $\begin{array}{l}\text { - I will try replacing a regular grain choice with couscous or } \\
\text { millet at a family meal. } \\
\text { - I will quiz } 3 \text { friends about the USDA MyPlate categories and } \\
\text { have them name } 3 \text { sources of protein. }\end{array}$ \\
\hline Lesson 8 & Family Fiesta & - No goal sheets \\
\hline
\end{tabular}

Weekly Lessons: Kitchen Portion

The kitchen portion of each lesson began with a demonstration of one or more of the cooking techniques that would be used for the recipe of the day. Either the Program Coordinator or Site Manager led the demo. After the demonstration the student participants took turns practicing the skill. Participants then self-selected into four groups; each group was situated at a cooking station supplied with all the necessary cooking equipment and utensils needed for the recipe provided. Each group of student 
participants then cooked the entire recipe from scratch. All food ingredients were located at a central "ingredient station" and the participants were responsible for measuring all ingredients according to the recipe. Students were also responsible for the cleanup process including washing dishes and cleaning the workstations. The Program Coordinator, Site Managers, and Health Ambassador volunteers acted as supervisors for each group and were responsible for operating certain kitchen appliances, such as the oven and stove, to ensure safety of the younger students. At the end of each kitchen portion of the lesson, all food was packed into to-go containers for the participants to take home. The participants were encouraged to share the meal as well as the recipes with their families in order to introduce new foods into the home and demonstrate the students' culinary abilities.

All recipes were selected by the Program Coordinator based on level of difficulty, types of ingredients, cooking techniques used, and relevancy to the middle school student population. Recipes were found online through web searches and most were modified slightly. Modifications were based on ingredient availability, ingredient nutritional value, available kitchen equipment and appliances, and group size. For example, the Quinoa Black Bean Salad recipe was adapted from its original by replacing certain ingredients with ones that are more widely available and familiar to adolescents (i.e. diced jalapeno peppers were replaced with diced bell peppers). All recipes were entered into a nutrient analysis program (www.nutritiondata.com) to produce a nutrition facts label. Table 2.2 provides the recipe schedule of Pink and Dude Chefs, Phase 2, according to the topic country. [See Appendix C for full recipes.] 
Table 2.2: Pink and Dude Chefs, Phase 2 recipe schedule.

\begin{tabular}{|l|l|l|}
\hline Lesson & Topic & Recipe \\
\hline Lesson 1 & Introduction / California Cuisine & $\begin{array}{l}\text { - Quinoa and Black Bean Salad } \\
\text { - Kale Chips (if time allows) }\end{array}$ \\
\hline Lesson 2 & France & - French Apple Tart \\
\hline Lesson 3 & Italy & $\begin{array}{l}\text { - Homemade Tomato Sauce } \\
\text { - Stuffed Manicotti }\end{array}$ \\
\hline Lesson 4 & Japan & $\begin{array}{l}\text { - Sushi Rice } \\
\text { - Cucumber and Avocado Sushi Rolls }\end{array}$ \\
\hline Lesson 5 & Greece & $\begin{array}{l}\text { - Greek Pita Pizzas } \\
\text { - Homemade Basil Pesto } \\
\text { - Homemade Pita Chips (if time allows) }\end{array}$ \\
\hline Lesson 6 & Thailand & $\begin{array}{l}\text { - Pad Thai } \\
\text { - Thai Mango Sticky Rice Dessert }\end{array}$ \\
\hline Lesson 7 & Morocco / Trivia & $\begin{array}{l}\text { - Moroccan Chicken } \\
\text { - Couscous }\end{array}$ \\
\hline Lesson 8 & Family Fiesta & $\begin{array}{l}\text { - Recipes vary. Typical menu includes Moroccan } \\
\text { Chicken, couscous, salad, and a fruit-based dessert. }\end{array}$ \\
\hline
\end{tabular}

\section{Family Fiesta}

The final lesson of Pink and Dude Chefs, Phase 2, called "Family Fiesta," was designed to be a culminating celebration to highlight the participants' accomplishments for their families and friends. Family Fiesta is an opportunity for the participants to display their culinary abilities, be individually recognized as a valued group member, and engage their family and friends in their success. Participants were responsible for the planning, preparation, and implementation of a three-course meal for up to 80 people. The chosen recipes were either identical to, or based on, recipes that participants had already cooked during the eight-week session. For example, the menu for the Fall 2013 Family Fiesta included Moroccan Chicken and an apple crisp (based on the French Apple Tart recipe cooked in class but adapted to a crisp to be easier to make for large numbers of people). Student participants were also in charge of serving the meal to the guests, which showcased their culinary achievements and inspired a sense of pride. This special occasion included decorations, photography, an awards ceremony, and a raffle to 
commend the participants who returned the most goal sheets. For a visual flowchart of the Pink and Dude Chefs program and the Phase 2 curriculum, please see Figure 2.1.

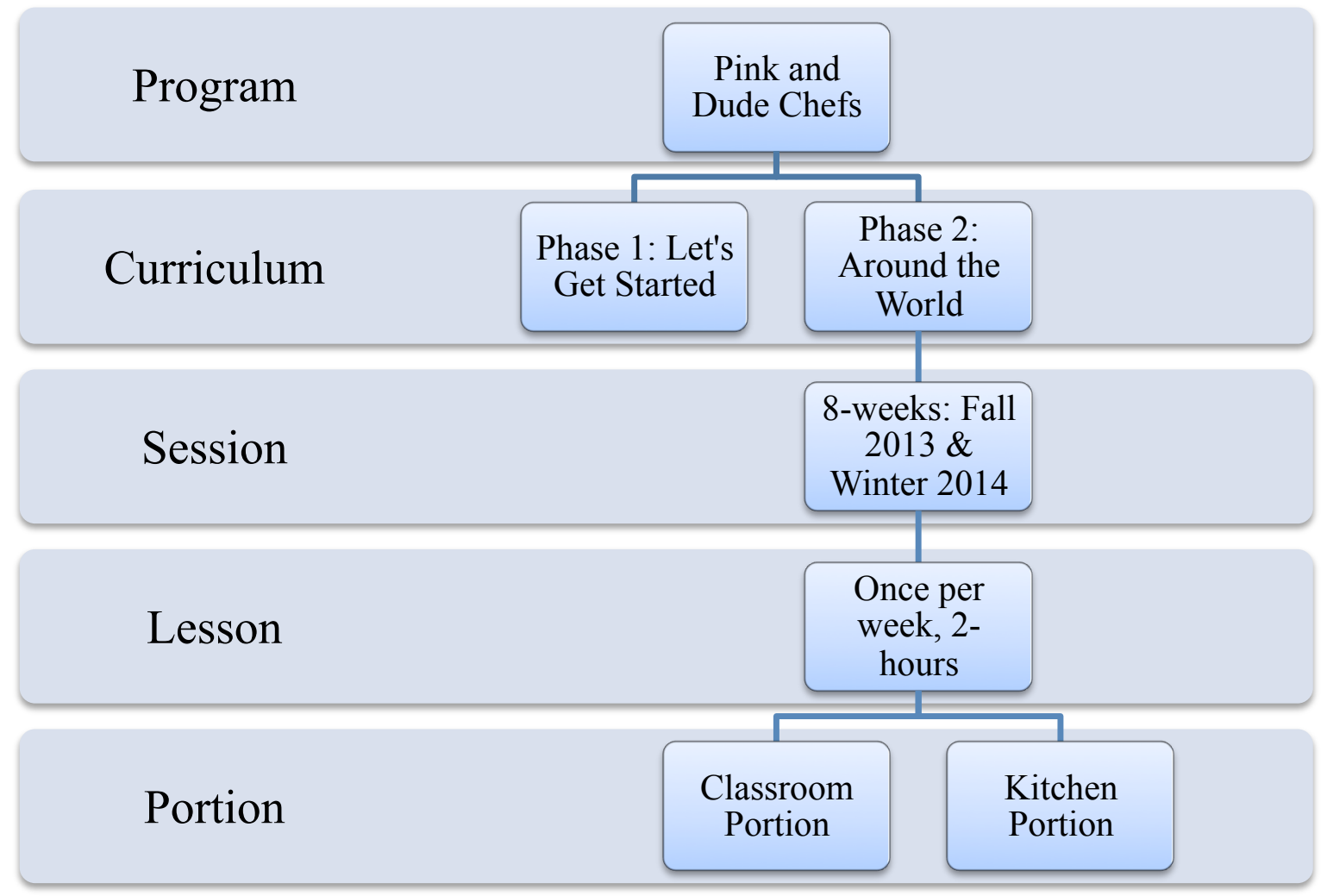

Figure 2.1: Flowchart depicting the structure of the Pink and Dude Chefs program and Phase 2 curriculum.

\section{Intervention Design}

\section{Study Site}

The current research evaluation of the Pink and Dude Chefs, Phase 2 program took place at Mesa Middle School in Arroyo Grande, California for both the Fall 2013 and Winter 2014 sessions. Arroyo Grande is located in San Luis Obispo County. The population of Arroyo Grande was 17,252 at the 2010 census, with a racial makeup of $85.3 \%$ non-Hispanic white, $15.7 \%$ Hispanic/Latino (Hispanics may be of any race, so are also included in applicable race categories), 3.4\% Asian, 0.9\% Black or African American, 0.7\% American Indian and Alaska Native, and 0.1\% Native Hawaiian and 
Other Pacific Islander (United States Census Bureau, 2014). According to the California Department of Education's Student Poverty - Free and Reduced Priced Meals (FRPM) dataset from 2012-2013, 60.71\% of students at Mesa Middle School are eligible to receive Free and Reduced Priced Meals (California Department of Education, 2014). Eligibility depends on household size (number of people) and is determined by a set income level. No income measure of the specific participants of the Pink and Dude Chefs Phase 2 program was included in this research.

\section{Participants}

Participants were $7^{\text {th }}$ and $8^{\text {th }}$ grade students enrolled in the Bright Futures afterschool program at Mesa Middle School. Bright Futures is an academic program funded by the After School Enrichment and Safety (AFES) state grant, and offers free admission to those students who fully participate. Students in this program are allowed to self-select into a variety of academic support and enrichment classes, including Pink and Dude Chefs. Therefore the participants of this study were determined through a convenience sample. A maximum of 15 students were allowed to participate in Pink and Dude Chefs each quarter due to space constraints in the kitchen. All participants were required to sign the Informed Assent Form and return the parent-signed Informed Permission Form. Excluded from the final sample were those participants who failed to submit required forms, did not complete both a pre-and post-survey, or did not attend at least $50 \%$ of the lessons. Participants from the Fall 2013 and Winter 2014 academic quarters were combined into one dataset. 


\section{Institutional Review Board Process}

Approval from the Institutional Review Board (IRB) at California Polytechnic State University is required for any research involving human subjects. The following forms were submitted and approved using the expedited review process:
A. Human Subjects Protocol Approval Form [Appendix D].
B. Human Subjects Research Protocol [Appendix E].
C. Informed Assent Form for Participant [Appendix F].
D. Informed Permission Form for Parents, in English and Spanish [Appendix G].
E. Pre- and Post-Intervention Survey [Appendix A].

\section{Survey}

A pre- and post-intervention survey was developed to measure each of five constructs addressed in this research: self-efficacy for cooking, cooking skills, nutrition knowledge, perceived social and family norms related to cooking, and outcome expectancies related to eating fruits and vegetables. Survey items were taken from previously validated assessment tools. Table 2.3 provides sample questions from each of the five constructs from the pre- and post-intervention survey. [For the full survey, see Appendix A].

Self-efficacy for cooking and cooking skills were measured using validated questions from the United Kingdom Food Standards Agency's CookWell program survey instrument. These items investigate how often participants cook at home, what kinds of cooking they normally do, the level of importance placed on cooking, and their confidence level for cooking skills such as reading a recipe and safely using a knife (Wrieden et al., 2007). 
Nutrition knowledge questions were developed for this project and were related to the USDA MyPlate 10 Tips Nutrition Education Series (United States Department of Agriculture, 2014). Participants were asked to fill in the name of each food group in the proper section of a blank MyPlate graphic and answer true-or-false questions related to nutrition behaviors.

Outcome expectancies related to fruit and vegetable consumption were measured using validated questions from the Compendium of Surveys for Nutrition Education and Obesity Prevention (California Department of Public Health, 2012) compiled by the Research and Evaluation Section of The Network for a Healthy California, a program within the California Department of Public Health. This is a compilation of validated surveys that are frequently used in public health nutrition education programs in California. The outcome expectancies questions were adapted from the "Power Play! School Idea and Resource Kit (SIRK) Survey," which was developed to assess the efficacy of a program that encourages fruit and vegetable consumption in low-income 911 year old children (Keihner et al., 2011). These questions explore the degree to which participants estimate that eating more fruits and vegetables leads to certain outcomes such as being healthier, thinking better in class, and having clearer skin.

Perceived social and family norms related to cooking were also measured using questions adapted from the Compendium of Surveys for Nutrition Education and Obesity Prevention. These questions were previously used in the "Gimme 5 Fruit, Juice, and Vegetables for Fun and Health" program evaluation to assess perceived social and family norms (Baranowski et al., 2000). For the Pink and Dude Chefs survey, the questions were 
designed to investigate the effect of peers and family on participants' cooking and attitudes and eating habits.

Table 2.3: Constructs and corresponding sample questions from pre- and postintervention survey.

\begin{tabular}{|c|c|}
\hline Construct & Sample Question \\
\hline $\begin{array}{l}\text { Self-efficacy } \\
\text { for Cooking }\end{array}$ & $\begin{array}{l}\text { - How confident are you that you can follow a simple recipe when } \\
\text { cooking? } \\
\text { a. Extremely confident } \\
\text { b. Somewhat confident } \\
\text { c. Confident } \\
\text { d. Not very confident } \\
\text { e. Not at all confident }\end{array}$ \\
\hline Cooking Skills & $\begin{array}{l}\text { - In a normal week, what kinds of cooking do you do at home? } \\
\text { a. Cook convenience foods and ready-made meals from a package } \\
\text { b. Put together ready-made ingredients to make a complete meal } \\
\text { c. Prepare dishes from basic ingredients, from scratch } \\
\text { d. Don't cook at all } \\
\text { e. Other, please explain }\end{array}$ \\
\hline $\begin{array}{l}\text { Nutrition } \\
\text { Knowledge }\end{array}$ & $\begin{array}{l}\text { - When eating a balanced meal, half of the food on my plate should be } \\
\text { fruits and vegetables. } \\
\text { a. True } \\
\text { b. False }\end{array}$ \\
\hline $\begin{array}{l}\text { Outcome } \\
\text { Expectancies }\end{array}$ & $\begin{array}{l}\text { - If I eat fruits and vegetables every day, I will have more energy. } \\
\text { a. I agree very much } \\
\text { b. I agree a little } \\
\text { c. I am not sure } \\
\text { d. I disagree a little } \\
\text { e. I disagree a lot }\end{array}$ \\
\hline $\begin{array}{l}\text { Perceived } \\
\text { Social Norms }\end{array}$ & $\begin{array}{l}\text { - What my friends think about cooking is important to me. } \\
\text { a. Agree strongly } \\
\text { b. Agree } \\
\text { c. Disagree } \\
\text { d. Disagree strongly }\end{array}$ \\
\hline $\begin{array}{l}\text { Perceived } \\
\text { Family Norms }\end{array}$ & $\begin{array}{l}\text { - Most people in my family think that eating 3-4 cups of fruits and } \\
\text { vegetables each day is: } \\
\text { a. A very good thing } \\
\text { b. A good thing } \\
\text { c. Not important } \\
\text { d. I don't know }\end{array}$ \\
\hline
\end{tabular}




\section{Data Collection}

The identical pre- and post-intervention survey was administered during the classroom portion on the first and last lessons of each Pink and Dude Chefs session. The Program Coordinator oversaw survey distribution and administration. A unique identification number was assigned to each student. This number was used to guarantee that each student's pre- and post-intervention surveys were correctly matched for data analysis. Students were informed that surveys would be kept confidential and they were instructed not to write their name on the surveys. Instructions were then given for completions of the remainder of the survey, and surveys were collected and returned to the Program Coordinator once finished.

\section{Data Analysis}

All data from the pre- and post-intervention surveys were entered into a Microsoft Access Database shortly after collection. To minimize error, the Program Coordinator and a research assistant separately entered the responses for double data entry. Data was then exported to a Microsoft Excel spreadsheet. The unique numerical code ensured that no identifying information was accessible.

Data was cleaned using a Microsoft Excel macro. The macro compared both sets of responses for the pre-intervention survey and the post-intervention survey and highlighted any existing discrepancies. Discrepancies (which mainly consisted of mistakes from data entry) were corrected by referring to the original surveys. After the data was cleaned, all participants who completed a pre-intervention survey but not a postintervention survey were removed from the dataset $(\mathrm{n}=6)$. This resulted in a sample size of 18 students, 11 of which participated in Fall 2013 and 7 of which participated in 
Winter 2014. There were two students who participated in Pink and Dude Chefs during both Fall 2013 and Winter 2014. Their pre- and post-intervention surveys from Winter 2014 were removed from the dataset because their scores may have been influenced by their prior participation. Only a student's first experience with Pink and Dude Chefs was of interest to address impact of the program. Therefore, the final sample size was 16 students: 11 from Fall 2013 and 5 from Winter 2014.

\section{Data Coding}

Data was coded by assigning a numerical value to each level of a question response. Table 2.4 provides an example of a coded survey question. Negative responses were assigned a lower number and positive responses assigned a high number. Therefore an increase in the numerical score indicates an increase in the positive valence of that response.

Table 2.4: Example coding of a survey question.

\begin{tabular}{|l|l|}
\hline $\begin{array}{l}\text { Question: If I eat fruits and vegetables every day, I will } \\
\text { have more energy. }\end{array}$ & Code \\
\hline I agree very much & 4 \\
\hline I agree a little & 3 \\
\hline I am not sure & 2 \\
\hline I disagree a little & 1 \\
\hline I disagree a lot & 0 \\
\hline
\end{tabular}

\section{Demographic Data}

Demographic data included the following variables: quarter of participation (Fall 2013 or Winter 2014), grade in school, age, sex, number of people living in the household (including the participant), race/ethnicity, and prior participation in a cooking class. Arbitrary numerical values were assigned for each of these variables. Upn cleaning the data it was observed that some participants answered demographic questions differently at pre-intervention and post-intervention (for example, participants' may have 
marked different answers for "race/ethnicity" on their pre- and post-intervention surveys). It was decided that these discrepancies would be resolved by using the preintervention answers.

Race/ethnicity was determined from the following question: "How would you describe yourself? (Circle ALL that apply to you)." Possible answers were American Indian or Alaska Native, Asian, Black or African American, Hispanic or Latino Mexican, Native Hawaiian or Other Pacific Islander, White/Caucasian, or Other (please fill in). It was determined that the primary racial groups of interest were Hispanic/Latino and "other," which included all other selections. Therefore each participant who selected "Hispanic/Latino" was assigned a value of 1, regardless of how many categories were selected. If "Hispanic/Latino" was not selected, that participant was assigned a score of 0 , regardless of how many categories were selected.

\section{Attendance and Goals Sheets}

The Program Coordinator logged each participant's attendance at the beginning of each lesson. Therefore each participant was assigned a numerical value indicating the total number of classes attended, out of 8 total per session. However, on several occasions participants attended the classroom portion and missed the kitchen portion or vise versa. For this reason attendance was not included as an explanatory variable in the multivariate statistical analysis (explained in further detail on page 61). The Program Coordinator also collected and logged all returned goal sheets. Goal sheets were distributed during lessons 1-7, and participants were instructed to complete and circle one of the two goals to receive credit. However, several participants in Fall 2013 and Winter 2014 turned in goal sheets with both goals circled (i.e. both the family goal and the friend 
goal were completed). Therefore in the analysis of perceived norms, the total number of goals completed and submitted would be used resulting in 14 possible per participant (two per seven lessons) with a maximum of 7 possible for either the family or for the peer group construct.

\section{Self-efficacy for Cooking}

Self-efficacy for cooking was measured by four survey questions designed to assess how confident participants felt about cooking from scratch, creating meals using new foods and ingredients, following a simple recipe, and using a knife when cooking. A response that demonstrated a greater degree of self-efficacy for cooking was represented with a higher score. Possible answers ranged from "Not at all confident," which was assigned a score of 0, to "Extremely confident," which was assigned a score of 4. The overall score for each question score was the numerical value of the answer chosen. Possible overall summed scores for self-efficacy for cooking ranged from 0 to 16 .

\section{Cooking Skills}

Cooking skills were measured by three survey questions designed to measure how often participants cook, what type of cooking they normally do, and for whom they normally cook. Decisions were made to determine which responses indicated increasing level of cooking skills. A response that demonstrated a greater degree of cooking skills was represented with a higher score.

The first cooking skills question asked "In a normal week, what kinds of cooking did you do at home? (Circle ALL that apply)." The answer "Don't cook at all" was assigned a score of 0 . The answer "Other, please explain" was assigned a score of 1 , after it was determined that each participant who chose to fill in this answer wrote that they 
"watch their parents cook." The answer "Cook convenience foods and ready-made meals from a package" was assigned a score of 2. The answer "Put together ready-made ingredients to make a complete meal" was assigned a score of 3 . The answer "Prepare dishes from basic ingredients, from scratch" was assigned a score of 4 . To determine the overall score for this question, it was decided that the highest level selected by the participant would be used; this assumes that a participant who knows how to prepare dishes from scratch is also competent in each lower level of cooking. Therefore, possible scores for this question ranged from 0 to 4.

The second cooking skills question asked "In a normal week, how often do you prepare and cook a meal from basic ingredients, from scratch? For example, making a meat and vegetable stir-fry starting with raw meat and fresh vegetables (Please circle only ONE)." The answer "Never" was assigned a score of 0 , the answer "Few days" was assigned a score of 1 , the answer "Most days" was assigned a score of 2 , and the answer "Every day" was assigned a score of 3. The overall score for the question was the numerical value of the answer chosen, which ranged from 0 to 3.

The third cooking skills question asked "In a normal week: (check ALL that apply)" followed by five responses: "a: I make meals for myself," "b: I make snacks for myself," "c: I make meals for others in my family," "d: I make snacks for others in my family," and "e: I did NOT make any meals or snacks for myself or for my family." The answers "a" and "c" were assigned a score of 2 , the answers "b" and " $d$ " were assigned a score of 1 , and the answer "e" was assigned a score of 0 . The overall score for the question represented the sum of each numerical response of the selected answers, which 
ranged from 0 to 6 . Therefore, possible overall summed scores for cooking skills ranged from 0 to 13 .

\section{Nutrition Knowledge}

Nutrition knowledge was measured by ten survey questions designed to assess knowledge gains related to USDA MyPlate. A response that demonstrated a greater degree of knowledge was represented with a higher score.

The first five questions asked participants to "Please fill in the names of the 5 food groups found on the USDA MyPlate." Participants were instructed to leave the line blank if they did not know the name of the group. A MyPlate graphic was provided with arrows pointing to each of the five categories. Therefore, a correct answer required the participant to write each food group in its correct location on the graphic. The five food groups were fruit, vegetables, dairy, grains, and protein. A blank response was assigned a score of 0 , a food group identified but incorrectly placed was assigned a score of 1 , and a correct response (food group correctly identified and placed) was assigned a score of 2. The overall score for each question was the numerical value of the answer chosen, which ranged from 0 to 2 .

The next four questions were "true or false" statements. The statements were: "Half the grains I eat should be whole grains," "When eating a balanced meal, half of the food on my plate should be fruits \& vegetables," "Eating too fast may lead to eating too much food," and "Eating while watching TV may lead to eating too much food." The correct answer for each question was "true." Therefore, a correct answer was assigned a score of 1 and an incorrect answer was assigned a score of 0 . The overall score for each question was the numerical value of the answer chosen, which ranged from 0 to 1. 
The last nutrition knowledge question asked participants "Which of the following foods are good sources of protein? (Please circle ALL that apply)." There were nine possible responses to this question, including meat, vegetables, eggs, fruits, beans \& peas, nuts \& seeds, seafood, dairy products, and tofu. Each of these answers, excluding vegetables and fruit, were regarded as a good source of protein; therefore, selecting these answers was a correct response. Additionally, not circling an incorrect answer (vegetables or fruit) was also considered a correct response. An incorrect response was defined as not circling a correct answer or circling an incorrect answer. Each correct response was assigned a score of 1 and each incorrect response was assigned a score of 0 . Participants received a net score based on their responses to each of the 9 answers. Possible scores ranged from 0 to 9 . Therefore, possible overall summed scores for nutrition knowledge ranged from 0 to 23 .

\section{Outcome Expectancies}

Outcome expectancies were measured by seven survey questions designed to assess the participants' beliefs about future outcomes of eating fruits and vegetables. A response that demonstrated a more positive outcome expectation was represented with a higher score. Each of the seven questions asked participants to choose how much they agreed with a statement about eating fruits and vegetables. An example question was "If $I$ eat fruits and vegetables every day, I will have more energy." Possible answers were "I agree very much," which was assigned a score of 4, "I agree a little," which was assigned a score of 3, "I am not sure," which was assigned a score of 2, "I disagree a little," which was assigned a score of 1 , and "I disagree very much," which was assigned a score of 0 . The overall score for each question was the numerical value of the answer chosen, which 
ranged from 0 to 4 . Therefore, possible overall summed scores for outcome expectancies ranged from 0 to 28 .

\section{Perceived Social and Family Norms}

Perceived social and family norms were measured by twelve survey questions designed to assess the influence of participants' family and friends on participants' attitudes about cooking and healthful eating. A response that demonstrated a greater degree of family or friend influence was represented with a higher score.

Questions were given in pairs. For example, in the first two questions asking participants how important it is to learn to cook, the first asked about cooking for their family and the second about cooking for their friends. Answers ranged from "Not at all important," which was assigned a score of 0, to "Extremely important," which was assigned a score of 4 . The overall score for each question was the numerical value of the answer chosen, which ranged from 0 to 4 . The remaining ten question pairs asked participants to choose how much they agreed with a statement related to their family or friends. Example questions were "I think cooking for my FAMILY is cool and fun" and "What my FRIENDS think about cooking is important to me." Possible answers were “Agree strongly," which was assigned a score of 3, "Agree," which was assigned a score of 2, "Disagree," which was assigned a score of 1, and "Strongly disagree," which was assigned a score of 0 . The overall score for each question was the numerical value of the answer chosen, which ranged from 0 to 3 . Therefore, possible overall summed scores for perceived social and family norms ranged from 0 to 38 . 


\section{Index Category}

An "index category" was created for each of the constructs by summing the numerical scores for all questions within each construct. For example, a "self-efficacy for cooking index" score was generated for each participant by totaling the scores of the four questions that measured self-efficacy. The purpose of this category was to provide an overall score for each construct to be analyzed pre- and post-intervention.

Perceived social and family norms questions were divided into two indices, with six questions each. The first was a compilation of all question scores that were related to peers (called "Social norms index") and the second was a compilation of all question scores that were related to family (called "Family norms index"). This resulted in a total of six index categories. See Table 2.5 for the index categories and their possible score ranges.

Table 2.5: Index categories and possible score ranges.

\begin{tabular}{|l|l|l|}
\hline Index Category & $\begin{array}{c}\text { Number of } \\
\text { Questions }\end{array}$ & $\begin{array}{l}\text { Possible Range } \\
\text { of Scores }\end{array}$ \\
\hline Self-efficacy for Cooking Index & 4 & $0-16$ \\
\hline Cooking Skills Index & 3 & $0-13$ \\
\hline Nutrition Knowledge Index & 10 & $0-23$ \\
\hline Outcome Expectancies Index & 7 & $0-28$ \\
\hline Social Norms Index & 6 & $0-19$ \\
\hline Family Norms Index & 6 & $0-19$ \\
\hline
\end{tabular}

\section{Statistical Analysis}

All analyses were performed using Microsoft Excel (Version 14.4.1) and JMP (SAS Institute, Version 10.0). For each pre-intervention and post-intervention survey question, the 16 participants' scores were summed and the mean was calculated. The mean was also calculated for each of the six index categories. A "difference" column for each survey question and each index category was created by calculating the post- 
intervention score minus the pre-intervention score. Therefore, a positive difference indicated an increase in score from pre-intervention to post-intervention.

The main outcome variables of interest in this study were the mean change in scores from pre- to post-intervention for each of the six index categories: self-efficacy for cooking, cooking skills, nutrition knowledge, perceived social norms, perceived family norms, and outcome expectancies. The main explanatory variable of interest was participation in the Pink and Dude Chefs, Phase 2 program. Potential confounding variables that may explain a portion of the results are quarter of participation (Fall 2013 or Winter 2014), sex, race/ethnicity (Hispanic or non-Hispanic), prior cooking class experience, and attendance.

\section{Pairwise Analysis}

One-sided paired t-tests were used to compare the mean score of each survey question and each index score pre- to post-intervention for cooking skills and nutrition knowledge. Because the literature provided strong evidence that cooking skills and nutrition knowledge would likely improve as a result of participation in a nutrition and culinary intervention, one-sided tests were appropriate. Two-sided paired t-tests were used to compare the mean score of each survey question and each index score pre- to post-intervention for self-efficacy for cooking, outcome expectancies, perceived social norms, and perceived family norms. Due to discrepancies and gaps in the literature regarding the effect of nutrition and culinary interventions on self-efficacy for cooking, outcome expectancies, and perceived social and family norms, two-sided tests were appropriate. There were 15 degrees of freedom and the significance level $(\alpha)$ was 0.05 . Normality tests were performed for each of the six index categories. 


\section{Multivariate Analysis}

Multivariate analysis of variance (MANOVA) was used to assess the overall effectiveness of the Pink and Dude Chefs, Phase 2 program and the potential effect of the demographic explanatory variables including: quarter participated (Fall 2013 or Winter 2014), sex, race, and prior cooking class experience. Attendance was not included as an explanatory variable because there were discrepancies regarding how attendance was recorded (i.e. on several occasions participants missed the classroom portion but attended the kitchen portion, or vice versa).

Because each of the index categories were on a different numerical scale (for example, the possible range of the cooking skills index scores was $0-13$ and the possible range of the outcome expectancies score was 1-28), an equation was used to create a "scaled" difference score for each of the index categories. 


\section{CHAPTER 3}

\section{Results}

\section{Demographics}

A total of 16 pairs of pre-intervention surveys and post-intervention surveys were included in the statistical analysis. These 16 pairs represented first-time exposures to Pink and Dude Chefs, as there were two students removed from the Winter 2014 dataset who had participated during both quarters. Demographic data was combined for Fall 2013 and Winter 2014 and also presented separately. See Table 3.1 for the baseline demographic data.

A majority of the participants were in $7^{\text {th }}$ grade $(68.8 \%)$. Most participants were either 12 years old $(43.8 \%)$ or 13 years old $(37.5 \%) .62 .5 \%$ of participants were female and $37.5 \%$ were male. Most participants $(62.5 \%)$ reported having 5 or more people in their household, including themselves. $62.5 \%$ of participants identified as Hispanic or Latino Mexican. Most of the participants $(81.2 \%)$ had no prior cooking class experience. The three students who did have prior cooking class experience included one that had participated in Pink and Dude Chefs the previous year (run by the same Program Coordinator but using the Phase 1 curriculum) and two that participated in other cooking programs. 
Table 3.1: Baseline demographic characteristics of Pink and Dude Chefs participants.

\begin{tabular}{|c|c|c|c|}
\hline Variable & $\begin{array}{l}\text { Total } \\
\text { n (\%) }\end{array}$ & $\begin{array}{l}\text { Fall } \\
2013 \\
\text { n (\%) }\end{array}$ & $\begin{array}{l}\text { Winter } \\
2014 \\
\mathrm{n}(\%)\end{array}$ \\
\hline & $\mathrm{n}=16^{1}$ & $\mathrm{n}=11$ & $\mathrm{n}=5$ \\
\hline \multicolumn{4}{|l|}{ Grade } \\
\hline $7^{\text {th }}$ grade & $11(68.8)$ & $7(63.6)$ & $4(80.0)$ \\
\hline $8^{\text {th }}$ grade & $5 \quad(31.2)$ & $4(36.3)$ & $1(20.0)$ \\
\hline \multicolumn{4}{|l|}{ Age } \\
\hline 12 & $7(43.8)$ & $5(45.5)$ & $2(40.0)$ \\
\hline 13 & $6(37.5)$ & $5(45.5)$ & $1(20.0)$ \\
\hline 14 & $3(18.8)$ & $1(9.1)$ & $2(40.0)$ \\
\hline \multicolumn{4}{|l|}{ Sex } \\
\hline Boy & $6(37.5)$ & $3(27.3)$ & $3(60.0)$ \\
\hline Girl & $10(62.5)$ & $8(72.7)$ & $2(40.0)$ \\
\hline \multicolumn{4}{|c|}{ Number of people living in household (including participant) } \\
\hline 4 & $6(37.5)$ & $3(27.3)$ & $3(60.0)$ \\
\hline 5 or more & $10(62.5)$ & $8(72.7)$ & $2(40.0)$ \\
\hline \multicolumn{4}{|l|}{ Race/ethnicity } \\
\hline Hispanic/Latino & $10(62.5)$ & $5(45.5)$ & $5(100)$ \\
\hline Other & $6(37.5)$ & $6(54.5)$ & -- \\
\hline \multicolumn{4}{|c|}{ Prior participation in a cooking class } \\
\hline No & $13(81.2)$ & $8(72.7)$ & $5(100)$ \\
\hline Yes & $3(18.8)$ & $3(27.3)$ & -- \\
\hline
\end{tabular}

\section{Attendance}

Overall, 15 of the 16 participants (93.8\%) attended at least $75 \%$ of the lessons (at least 6 out of 8), demonstrating good overall attendance. One participant attended 5 out of the 8 lessons. During Fall 2013 the majority of participants attended all 8 lessons (63.6\%). During Winter 2014 the majority of participants attended at least 7 out of 8 lessons (80.0\%). Table 3.2 shows participant attendance for Pink and Dude Chefs. 
Table 3.2: Participant Attendance for Pink and Dude Chefs, Phase 2.

\begin{tabular}{llll}
\hline Number of Classes Attended $^{1}$ & $\begin{array}{l}\text { Total } \\
\mathrm{n}(\%)\end{array}$ & $\begin{array}{l}\text { Fall 2013 } \\
\mathrm{n}(\%)\end{array}$ & $\begin{array}{l}\text { Winter 2014 } \\
\mathrm{n}(\%)\end{array}$ \\
\hline & $\mathrm{n}=16$ & $\mathrm{n}=11$ & $\mathrm{n}=5$ \\
\hline 8 & $8(50.0)$ & $7(63.6)$ & $1(20.0)$ \\
7 & $6(37.5)$ & $3(27.3)$ & $3(60.0)$ \\
6 & $1(6.25)$ & $1(9.09)$ & -- \\
5 & $1(6.25)$ & -- & $1(20.0)$ \\
4 or fewer & -- & -- & -- \\
\hline${ }^{1}$ Out of 8 classes total per session. & &
\end{tabular}

\section{Goal Sheets}

Overall a high number of goals were completed. Over both sessions 13 out of 16 students (81.2\%) completed at least one goal and only 3 did not complete any goals (18.8\%). A total of 58 goals were completed by 13 students. Of those completed, 28 were friend goals (48.3\%) and 30 were family goals (51.7\%). Eight students completed both family and friend goals. Of the goals complete by those eight students alone, 24 were friend goals (46\%) and 28 were family goals (54\%). Of the five students completing only friend goals or family goals alone, 2 were friend goals and 3 were family goals. Figure 3.1 shows the total number of goal sheets submitted by participant and the ratio of family goals to friend goals. 


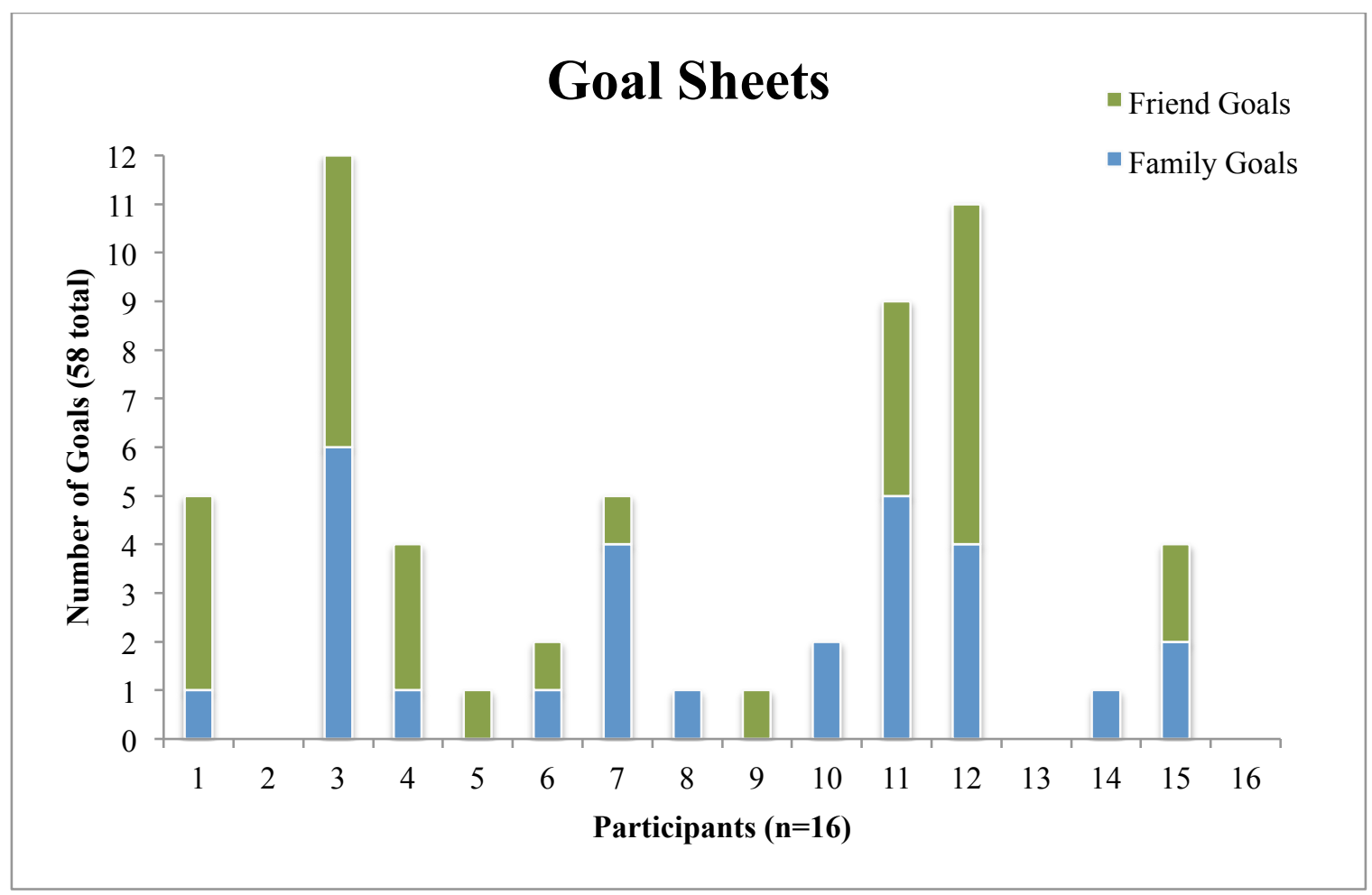

Figure 3.1: Total number of goal sheets submitted by participant. Column colors indicate the ratio of family goals to friend goals completed per individual.

Pairwise Analysis of Change

\section{Self-efficacy for Cooking}

Research question \#1 asks: Does participation in the Pink and Dude Chefs, Phase 2 program influence self-efficacy for cooking? Results show that there was no significant difference between the mean self-efficacy index score from pre- to post-intervention. The mean self-efficacy index score decreased from 13.5 to $13.3(P=0.73)$. One of the four questions within the self-efficacy for cooking section showed a non-significant increase: confidence in the ability to follow a simple recipe increased from 3.56 to $3.69(P=0.58)$. Table 3.3 shows the mean self-efficacy for cooking scores pre- and post-intervention and the change in mean self-efficacy for cooking scores. 
Table 3.3: Mean scores for self-efficacy for cooking and change in mean score from preto post-intervention $(\mathrm{n}=16)$.

\begin{tabular}{lllll}
\hline \multirow{2}{*}{ Self-efficacy for Cooking Question } & $\begin{array}{l}\text { Mean Pre- } \\
\text { score }\end{array}$ & $\begin{array}{l}\text { Mean Post- } \\
\text { score }\end{array}$ & $\begin{array}{l}\text { Change in } \\
\text { Mean }\end{array}$ & $\begin{array}{l}\text { P- } \\
\text { value }^{1}\end{array}$ \\
\hline 1. Cooking from scratch & 3.00 & 2.88 & -0.13 & 0.54 \\
2. Cooking with new foods/ingredients & 3.06 & 2.94 & -0.13 & 0.67 \\
3. Following a simple recipe & 3.56 & 3.69 & 0.13 & 0.58 \\
4. Safely using a knife while cooking & 3.88 & 3.81 & -0.06 & 0.72 \\
Self-efficacy for cooking index & 13.5 & 13.3 & -0.19 & 0.73 \\
\hline
\end{tabular}

${ }^{\mathrm{T}} \mathrm{P}$-value from two-sided paired t-tests, $p<0.05$.

${ }^{2}$ Self-efficacy for cooking index comprised of sum of 4 self-efficacy for cooking questions (range 0 to 16 ).

\section{Cooking Skills}

Research question \#2 asks: Does participation in the Pink and Dude Chefs, Phase

2 program increase cooking skills? Overall, the mean cooking skills index score

significantly increased from 5.75 to $6.94(P=0.02)$. Each of the three questions within the cooking skills section showed an increase in score. The third question, which asked the participant who they normally cooked for at home and whether they made meals or snacks, increased significantly $(P=0.02)$. Table 3.4 shows the mean cooking skills scores pre- and post-intervention and the change in mean cooking skills scores.

Table 3.4: Mean scores for cooking skills and change in mean score from pre- to postintervention $(\mathrm{n}=16)$.

\begin{tabular}{lllll}
\hline Cooking Skills Question & $\begin{array}{l}\text { Mean Pre- } \\
\text { score }\end{array}$ & $\begin{array}{l}\text { Mean Post- } \\
\text { score }\end{array}$ & $\begin{array}{l}\text { Change in } \\
\text { Mean }\end{array}$ & P-value $^{1}$ \\
\hline 1. Type of cooking at home & 2.63 & 2.81 & 0.19 & 0.27 \\
2. Frequency of cooking from scratch & 1.06 & 1.38 & 0.31 & 0.07 \\
3. Who participant cooked for & 2.06 & 2.75 & 0.69 & $0.02^{*}$ \\
Cooking skills index $^{2}$ & 5.75 & 6.94 & 1.19 & $0.02^{*}$ \\
\hline
\end{tabular}

${ }^{\mathrm{I}} \mathrm{P}$-value from one-sided paired t-tests, $p<0.05$.

${ }^{2}$ Cooking skills index comprised of sum of 3 cooking skills questions (range 0 to 13 ).

\section{Nutrition Knowledge}

Research question \#3 asks: Does participation in the Pink and Dude Chefs, Phase 2 program increase nutrition knowledge related to the USDA MyPlate? Overall, the mean nutrition knowledge index score significantly increased from 9.38 to 18.9 
$(P<0.0001)$. Each of the ten questions within the nutrition knowledge section showed an increase in score, and seven of these were significantly higher. Each of the five MyPlate category questions increased significantly $(P<0.0001)$ demonstrating improved knowledge of MyPlate at the end of the intervention. Of the four true or false questions, only the question that asked whether eating while watching TV may lead to eating too much food significantly increased $(P<0.01)$. Understanding food sources of protein was also improved as seen by a significant increase in correct responses to the question that asked participants which of nine foods listed were good sources of protein $(P<0.02)$.

Table 3.5 shows the mean nutrition knowledge scores pre- and post-intervention and the change in mean nutrition knowledge scores.

Table 3.5: Mean scores for cooking skills and change in mean score from pre- to postintervention $(\mathrm{n}=16)$.

\begin{tabular}{llllc}
\hline Nutrition Knowledge Question & $\begin{array}{l}\text { Mean Pre- } \\
\text { score }\end{array}$ & $\begin{array}{l}\text { Mean Post- } \\
\text { score }\end{array}$ & $\begin{array}{l}\text { Change in } \\
\text { Mean }\end{array}$ & P-value $^{1}$ \\
\hline 1. MyPlate category: Fruit & 0.38 & 1.94 & 1.56 & $<0.0001^{*}$ \\
2. MyPlate category: Vegetables & 0.25 & 2.00 & 1.75 & $<0.0001^{*}$ \\
3. MyPlate category: Dairy & 0.44 & 2.00 & 1.56 & $<0.0001^{*}$ \\
4. MyPlate category: Grains & 0.44 & 1.69 & 1.25 & $<0.0001^{*}$ \\
5. MyPlate category: Protein & 0.25 & 1.69 & 1.44 & $<0.0001^{*}$ \\
6. True or False: Whole grains & 0.88 & 0.88 & 0.00 & 0.50 \\
7. True or False: Fruit \& vegetables & 0.88 & 1.00 & 0.13 & 0.08 \\
8. True or False: Eating too fast & 0.88 & 1.00 & 0.13 & 0.08 \\
9. True or False: Watching TV & 0.50 & 0.81 & 0.31 & $0.01^{*}$ \\
10. Food sources of protein & 4.50 & 5.89 & 1.38 & $0.02^{*}$ \\
Nutrition knowledge index $^{2}$ & 9.38 & 18.9 & 9.50 & $<0.0001^{*}$ \\
\hline
\end{tabular}

${ }^{\mathrm{I}} \mathrm{P}$-value from one-sided paired t-tests, $p<0.05$.

${ }^{2}$ Nutrition knowledge index comprised of sum of 10 nutrition knowledge questions (range 0 to 23).

\section{Outcome Expectancies}

Research question \#4 asks: Does participation in the Pink and Dude Chefs, Phase 2 program influence outcome expectancies related to eating fruits and vegetables?

Overall, there was no significant change between the outcome expectancies index score from pre- to post-intervention. The mean outcome expectancies index score decreased 
from 25.5 to $24.9(P=0.52)$. One of the seven questions within the cooking skills section showed a non-significant increase: the expectancy of becoming stronger from eating fruits and vegetables increased from 3.63 to $3.69(P=0.67)$. Table 3.6 shows the mean outcome expectancies scores pre- and post-intervention and the change in mean outcome expectancies scores.

Table 3.6: Mean scores for outcome expectancies and change in mean score from pre- to post-intervention $(\mathrm{n}=16)$.

\begin{tabular}{lllcl}
\hline Outcome Expectancies Question & $\begin{array}{l}\text { Mean Pre- } \\
\text { score }\end{array}$ & $\begin{array}{l}\text { Mean Post- } \\
\text { score }\end{array}$ & $\begin{array}{l}\text { Change in } \\
\text { Mean }\end{array}$ & P-value $^{1}$ \\
\hline 1. I will become stronger & 3.63 & 3.69 & 0.06 & 0.67 \\
2. My friends will start eating them too & 3.00 & 2.88 & -0.13 & 0.61 \\
3. I will have more energy & 3.81 & 3.75 & -0.06 & 0.75 \\
4. I will be healthier & 4.00 & 3.94 & -0.06 & 0.33 \\
5. My family will be proud of me & 3.63 & 3.63 & 0.00 & 1.00 \\
6. I will think better in class & 3.81 & 3.50 & -0.31 & 0.21 \\
7. I will have a nicer smile and clear skin & 3.63 & 3.56 & -0.06 & 0.75 \\
Outcome expectancies index $^{2}$ & 25.5 & 24.9 & -0.56 & 0.52 \\
\hline
\end{tabular}

${ }^{1} \mathrm{P}$-value from two-sided paired t-tests, $p<0.05$.

${ }^{2}$ Outcome expectancies index comprised of sum of 7 outcome expectancies questions (range 0 to 28).

\section{Perceived Social and Family Norms}

Research question \#5 asks: Does participation in the Pink and Dude Chefs, Phase 2 program influence perceived social and family norms related to cooking? Overall, the mean social norms index score increased from 12.8 to $13.1(P=0.41)$ and the family norms index score increased from 15.1 to $15.8(P=0.08)$. Eight of the thirteen questions within the perceived social and family norms section showed an increase in score, but none of these were statistically significantly different. Table 3.7 shows the mean social and family norms scores pre- and post-intervention and the change in mean social and family norms scores.

Research question \#6 asks: Are either perceived social norms or perceived family norms more highly associated with the eating/culinary behaviors of participants of Pink 
and Dude Chefs, Phase 2? The family norms index score was significantly greater than the social norms index score at both pre-intervention $(P=0.0029)$ and post-intervention $(P=0.0058)$. The increase in the family norms index score $(0.63)$ was greater than the increase in the social norms index (0.38) score from pre- to post-intervention.

Furthermore, for each of the six question pairs, the mean pre- and post-intervention score was higher for family norms than social norms.

Table 3.7: Mean scores for perceived social and family norms and change in mean score from pre- to post-intervention ( $\mathrm{n}=16)$.

\begin{tabular}{|c|c|c|c|c|}
\hline Social and Family Norms Question & $\begin{array}{l}\text { Mean Pre- } \\
\text { score }\end{array}$ & $\begin{array}{l}\text { Mean Post- } \\
\text { score }\end{array}$ & $\begin{array}{l}\text { Change in } \\
\text { Mean }\end{array}$ & P-value ${ }^{1}$ \\
\hline Social norms: learning to cook & 3.13 & 3.00 & -0.13 & 0.58 \\
\hline Social norms: cooking fun and cool & 2.25 & 2.06 & -0.19 & 0.27 \\
\hline Social norms: cooking importance & 1.94 & 1.88 & -0.06 & 0.72 \\
\hline Social norms: fruits and vegetables & 1.25 & 1.75 & 0.50 & 0.07 \\
\hline Social norms: eating healthy food & 2.00 & 2.06 & 0.06 & 0.67 \\
\hline Social norms: exercising & 2.19 & 2.38 & 0.19 & 0.19 \\
\hline Family norms: learning to cook & 3.38 & 3.25 & -0.13 & 0.61 \\
\hline Family norms: cooking fun and cool & 2.75 & 2.75 & 0.00 & 1.00 \\
\hline Family norms: cooking importance & 2.38 & 2.44 & 0.06 & 0.67 \\
\hline Family norms: fruits and vegetables & 1.94 & 2.38 & 0.44 & 0.11 \\
\hline Family norms: eating healthy food & 2.38 & 2.50 & 0.13 & 0.33 \\
\hline Family norms: exercise & 2.31 & 2.44 & 0.13 & 0.33 \\
\hline Social norms index ${ }^{2}$ & 12.8 & 13.1 & 0.38 & 0.41 \\
\hline Family norms index ${ }^{3}$ & 15.1 & 15.8 & 0.63 & 0.08 \\
\hline
\end{tabular}

${ }^{\mathrm{I}} \mathrm{P}$-value from two-sided paired t-tests, $p<0.05$.

${ }^{2}$ Social Norms Index comprised of sum of 6 social norms questions (range 0 to 19).

${ }^{3}$ Family Norms Index comprised of sum of 6 social norms questions (range 0 to 19).

\section{Index Score Summary}

Table 3.8 shows a summary of the mean scores for each of the six index categories pre- and post-intervention and the change in mean index score. The mean cooking skills index score $(P=0.02)$ and the mean nutrition knowledge index score $(P<0.0001)$ significantly increased from pre- to post-intervention. Figure 3.2 shows change in the mean index score from pre-intervention to post-intervention. 
Table 3.8: Mean scores for index categories and change in mean index score from pre- to post-intervention $(n=16)$.

\begin{tabular}{llllc}
\hline \multirow{2}{*}{ Index Category } & $\begin{array}{l}\text { Mean Pre- } \\
\text { score (SD) }\end{array}$ & $\begin{array}{l}\text { Mean Post- } \\
\text { score (SD) }\end{array}$ & $\begin{array}{l}\text { Change in } \\
\text { Mean (SD) }\end{array}$ & P-value \\
\hline Self-efficacy for Cooking $^{1}$ & 13.5 & 13.3 & -0.19 & 0.73 \\
Cooking Skills $^{2}$ & 5.75 & 6.94 & 1.19 & $0.02^{*}$ \\
Nutrition Knowledge $^{2}$ & 9.38 & 18.9 & 9.50 & $<0.0001^{*}$ \\
Outcome Expectancies $^{1}$ & 25.5 & 24.9 & -0.56 & 0.52 \\
Social Norms $^{1}$ & 12.8 & 13.1 & 0.38 & 0.41 \\
Family Norms $^{1}$ & 15.1 & 15.8 & 0.63 & 0.08 \\
\hline
\end{tabular}

${ }^{1} \mathrm{P}$-value from two-sided paired t-tests, $p<0.05$.

${ }^{2} \mathrm{P}$-value from one-sided paired t-tests, $p<0.05$.

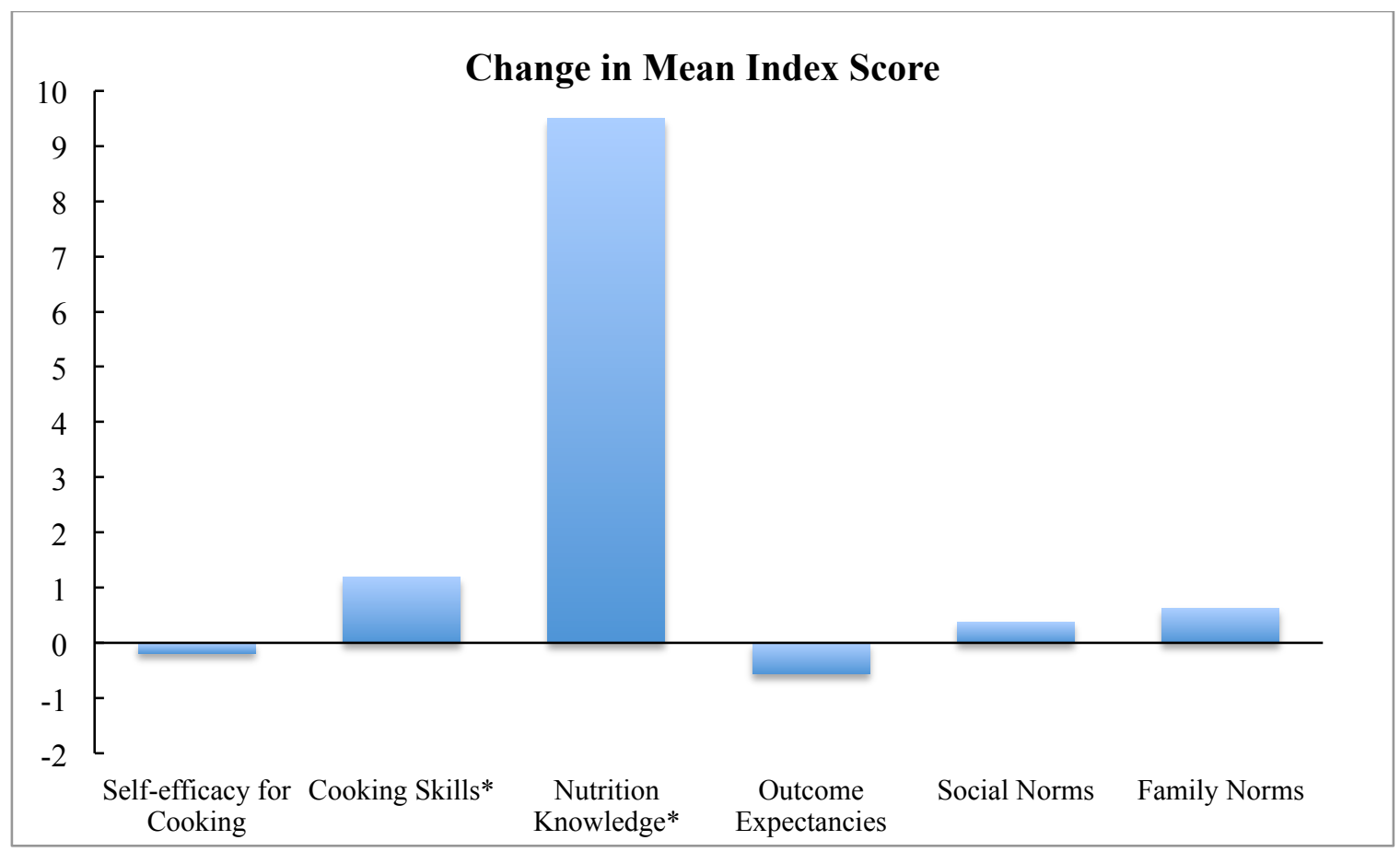

Figure 3.2: Change in mean index scores from pre- to post-intervention.

*Statistically significant difference, $p<0.05$.

\section{Multivariate Analysis Results}

MANOVA was conducted including quarter of participation, sex, race, and prior cooking class experience as the explanatory variables and the change in each of the six index scores as the outcome variables. Results from this MANOVA showed that overall, the Pink and Dude Chefs, Phase 2 program demonstrated a significant multivariate effect on the six outcome variables (exact $\mathrm{F}=16.43 ; 6 \mathrm{df} ; P=0.0038$ ). Overall there was no 
significant confounding effect from quarter of participation $(P=0.71)$, sex $(P=0.50)$, race $(P=0.57)$, and prior class cooking experience $(P=0.72)$.

Univariate results showed that significant effects on nutrition knowledge persisted $(P<0.0001)$. Non-significant effects were detected for self-efficacy for cooking $(P=0.37)$, cooking skills $(P=0.57)$, outcome expectancies $(P=0.72)$, perceived social norms ( $P=0.70)$, and perceived family norms $(P=0.15)$. However, the multivariate analysis no longer indicated a statistically significant effect on cooking skills $(P=0.57)$ when controlling for the explanatory variables, an effect that was seen in the pairwise analysis $(P=0.03)$.

The quarter of participation was significantly associated with the change in nutrition knowledge; those who participated during Winter 2014 showed a greater change in score $(P=0.04)$. Prior cooking class experience was also significantly associated with the change in nutrition knowledge; those with a prior class experience showed a greater change in score $(P=0.03)$. 


\section{CHAPTER 4}

\section{Discussion}

This study assessed the effectiveness of Pink and Dude Chefs, Phase 2, a multicultural-themed nutrition and culinary intervention targeted towards middle school students in Arroyo Grande, California. Results indicated that the program resulted in a significant improvement in nutrition knowledge and cooking skills of participants, but did not significantly influence self-efficacy for cooking, outcome expectancies related to eating fruits and vegetables, or perceived social and family norms related to cooking and eating healthfully.

Nutrition knowledge related to USDA MyPlate significantly increased after participation in the Pink and Dude Chefs, Phase 2 program. Each of the five questions in which participants identified the MyPlate food categories improved significantly. The design of Phase 2 emphasized cultural foods and recipe ingredients by MyPlate food groups. By comparing the staple foods of each topic country to the MyPlate categories, participants were able to apply knowledge concepts on a weekly basis. After seven weeks participants also demonstrated a significant increase in their knowledge of food sources of protein. In-class discussions about the food culture of each topic country frequently addressed the wide variety of protein-rich foods. Positive responses to the question whether or not eating while watching TV leads to eating too much food also significantly increased. Interestingly, this concept was not directly addressed during the session; possibly the participants inferred the knowledge through in-class discussions about the importance of leading an active lifestyle. Another consideration is that the nutrition knowledge survey questions were not previously validated, as were the rest of the survey 
questions. However, the participants seemed to have no problem understanding these questions.

Improvement in nutrition knowledge is a key finding in this current research. Knowledge is an important construct of behavioral change theories because it increases the likelihood of a healthy behavior occurring (Glanz et al., 2008). However, knowledge alone is not a single predictor because it interacts with other mediators to encourage behavior change. Furthermore, several studies have found a significant positive relationship between nutritional knowledge and self-efficacy for adopting healthy behaviors (Rabiei, Sharifirad, Azadbakht, \& Hassanzadeh, 2013). Potentially the combination of knowledge and skill components emphasized in the Pink and Dude Chefs, Phase 2 program will allow participants to apply the USDA MyPlate guidelines to actual daily meal preparation techniques in the future. A previous intervention that combined nutrition education with culinary skill building found significant positive outcomes for nutrition knowledge, culinary self-efficacy, and preference for plant foods after 10 lessons (Liquori et al., 1998).

The multivariate analyses showed that nutrition knowledge improvements were significantly associated with quarter of participation and prior cooking class experience. Participants in the Winter 2014 session showed a significantly greater score increase than those in the Fall 2013 session. This may have been due to the Program Coordinator's teaching skills improving over time, or that the smaller group during the Winter session caused participants to partake in class discussions more often. Another possible explanation is that during the winter quarter there were two students who had also participated during the fall session. They may have imparted some level of influence on 
the other participants, even though they were not included in the statistical analysis. However, the small sample size in Winter $2014(\mathrm{n}=5)$ makes it difficult to draw conclusions about differences between the quarter of participation. In addition, this result may have been due to chance. Participants with prior cooking class experience also showed a significantly greater score increase than those without a prior cooking class experience. This may be because prior cooking classes also emphasized nutrition knowledge concepts such as USDA MyPlate and food groups, or this result may be due to chance.

Cooking skills also significantly increased from pre- to post-intervention according to the paired t-tests results. However, after controlling for quarter of participation, sex, race, and prior cooking experience in the multivariate analyses, the significant effect did not persist. The impact of the covariates on the variance explained by the changes in cooking skills is difficult to interpret, potentially because of the small sample size. Regardless, there was a notable increase in the mean cooking skills index score after participation in Pink and Dude Chefs, Phase 2. Weekly repetition of basic culinary skills such as chopping, dicing, measuring, mixing, and recipe-reading allowed participants to hone their skills while receiving assistance and encouragement from the staff. Specifically, participants reported a significant increase in frequency regarding who they normally cooked for at home and whether they made meals or snacks at home. The weekly goal sheets and recipe cards made it possible for participants to practice their newly acquired skills in the home and this likely contributed to the increased amount of cooking meals and snacks at home. The participants verbally expressed enthusiasm during the program about recreating the recipes from Pink and Dude Chefs, Phase 2 for 
their families, which seemed to instill a sense of pride. Improving skills directly increases the likelihood of a behavior occurring (Glanz et al., 2008), and studies have consistently shown that food prepared from scratch in the home environment tends to be healthier than food consumed away from the home (Larson et al., 2006). Therefore the increase in cooking skills seen in this program may be an indicator that participants have begun to make healthy changes in the home environment.

There were only three questions used to assess cooking skills and these questions did not assess actual skills (i.e. observing the participant use a knife). A true measurement of cooking skills cannot be done on a survey, which contributes to the lack of clear evidence as to how cooking skills influence one's dietary behavior (Hartmann et al., 2013). Survey measurements of cooking skills may be subjected to social, cultural, and personal influences, and research investigating cooking skills through direct observation is lacking (Hartmann et al., 2013) Future research into creating a more reliable and applicable cooking skills survey measure may be more effective as a means of determining how improved cooking skills may relate to improved eating behaviors.

Self-efficacy for cooking, outcome expectancies related to eating fruits and vegetables, and social and family norms did not significantly change after participation in Pink and Dude Chefs, Phase 2. In fact, the mean index scores for self-efficacy for cooking and outcome expectancies actually decreased from pre- to post-intervention. The questions within the self-efficacy for cooking survey measure asked participants how confident they felt cooking from scratch, cooking with new foods and ingredients, following a simple recipe, and safely using a knife. Considering the facts that the participants repeatedly practiced each of these tasks throughout the session and that 
cooking skills significantly increased, the decrease in self-efficacy is puzzling. The Chessen (2008) Pink Chefs investigation showed a statistically significant improvement in self-efficacy for cooking, but with a 16-lesson twice-weekly version of the program. Twice-weekly exposures may be more effective than one-weekly exposures to improve newly acquired skills. Similar nutrition and culinary interventions have generally found positive outcomes for self-efficacy for cooking (Halford et al., 2013; Liquori et al., 1998; Cunningham-Sabo \& Lohse, 2014), however these studies had much larger sample sizes than the current research. One potential reason for the decrease in mean index score is that many of the participants with prior at-home cooking experience felt very confident in their abilities at the time of the pre-survey, only to realize that cooking in a different environment (i.e. groups of peers with guidance from college students) slightly lessened their confidence. Another possibility is that this population of middle school students (living in an agriculturally-rich, predominantly Hispanic region of California) simply has more exposure to cooking at home than the general population. With such a small sample size, finding change in a group with initially positive scores is difficult.

Outcome expectancies also slightly decreased slightly from pre- to postintervention. Of the seven questions which asked participants to choose the degree to which they agreed with statements about the outcomes of eating fruits and vegetables every day, five showed a decrease in score. Though outcome expectancies have been shown to directly correlate to eating behaviors in adults, the same is not true for children, and the effect of nutrition and culinary interventions on outcome expectancies is not consistent in the literature (McClain et al., 2009). The concepts suggested in the outcome expectancies survey questions were not consistently directly addressed in the curriculum 
of Pink and Dude Chefs, Phase 2. Rather the concepts were embedded into the multicultural component through examining the connection between dietary patterns and health outcomes of each topic country. For example, the participants learned that people living in Japan tend to consume a diet rich in plant foods, and subsequently these people tend to have very low rates of heart disease and cancer and have a high life expectancy. Participants were then asked to draw conclusions about this relationship, which often led to a discussion about the importance of eating fruits and vegetables. While these discussions may have impacted their beliefs about the outcomes of eating fruits and vegetables, they may not have applied to the specific outcomes addressed in the survey questions (i.e. If I eat fruits and vegetables every day, I will think better in class). Further research is needed to explore both the impact of nutrition and culinary interventions on outcome expectancies and the relationship between outcome expectancies and dietary habits in adolescents.

Perceived social and family norms both increased post-intervention but neither increase was statistically significant. Family norms scores were consistently found to be higher than scores for social norms, potentially surprising in a teenaged population. The social and family norms survey questions focused on injunctive perceived norms (beliefs about what friends and family believe) and normative beliefs (beliefs about the extent to which friends and family think the subject should or should not perform a particular behavior). Both peer and family influences have been shown to be important determinants of behaviors such as healthful eating (Ball et al., 2010; Fitzgerald et al., 2013). Although there is strong evidence that peer and family norms may directly influence dietary behaviors, very little research has examined the relationship between 
peer and family norms and culinary behaviors (Nelson et al., 2013). A goal of the current research was to examine whether a nutrition and culinary intervention with a group of peers may cause participants to adopt more positive beliefs about how their friends and family perceive cooking and eating healthfully, which may in turn lead to behavioral changes in the home and at school.

The group-work component of Pink and Dude Chefs, Phase 2 program emphasized the importance of teamwork and allowed participants a chance for observational leaning from their peers. Furthermore, the weekly goal sheets and recipe cards gave participants a chance to practice the skills and knowledge learned in the program with their family and friends. However, the fact that the program did not significantly influence social and family norms is not surprising, perhaps due to the short duration (eight weeks) and once weekly lessons. Normative beliefs are often deeply rooted and may be difficult to change in a short period of time. Another consideration is that the post-intervention surveys were administered just before the Family Fiesta celebration. Had participants answered these questions afterwards, responses may have reflected any positive influence Family Fiesta had on participants' perceptions of their families' reception to cooking. The increase in mean index scores for both social and family norms may indicate a slight shift towards more positive beliefs about how friends and family perceive cooking and eating healthfully, but further research is needed to explore how culinary programs influence normative beliefs.

Potentially of greater interest was whether peers or family were a stronger influential motivator towards healthy eating and cooking practices for the participants. At pre-intervention, the mean score for each question pair was higher for perceived family 
norms than for perceived social norms. For example, the mean score for the question that asked participants how important it is to learn to cook for their friends was 3.13 and for family was 3.38 . The mean index scores at pre-intervention were 12.8 for social norms and 15.1 for family norms, a statistically significantly higher difference. The same was true at post-intervention; the mean score for each question pair was higher for family norms and for social norms and the mean index score for family norms was significantly higher than the mean index score for social norms. These results suggest that in this population of middle school students, family may have a stronger influence on behaviors such as cooking and eating healthfully than peers do. Middle school students may perceive cooking to be an activity that takes place at home in a family context, which causes them to be less concerned about what their peers think about cooking. Participants also perceived that their family placed greater value on eating fruits and vegetables every day and exercising than their peers did. Thus, an implication for future nutrition and culinary interventions is a need to improve curriculum design by incorporating greater family involvement. This may be achieved through pre-intervention parent meetings, including parents in one or more lessons, additional take-home recipes and activities, and greater parent involvement at the Family Fiesta celebration.

Weekly goal sheets were designed as a take-home component of Pink and Dude Chefs, Phase 2. The purpose was to extend learning beyond the program and encourage participants to share their newly acquired knowledge and skills with their friends and family. Goal sheets were incentivized through raffle tickets for the Family Fiesta celebration. Of the 58 goals completed throughout both sessions, the proportion of friendbased goals and family-based goals were close to equal (48.3\% and 51.7\%, respectively). 
An original hypothesis was that an analysis of goal sheet selections (family-based activities versus friend-based activities) might help to explain whether perceived social norms or perceived family norms were more highly associated with the eating/culinary behaviors of participants. However, this relationship turned out to be difficult to assess in this dataset for several reasons. First, different participants completed the goal sheets in different ways. The instructions asked them to circle and complete one of the two goals with the intent of having each participant make a choice of completing one weekly goal with either peers or with family. Instead multiple students completed both the peer and the family activities on the goal sheet over the weeks of the program. To accommodate how the students were using the goal sheets, the Program Coordinator announced in class that they were encouraged to complete both. Many students, however, continued to follow the directions and complete just one goal. The participants' reasoning behind circling both goals may have had more to do with the raffle ticket incentive than any regard to family and social considerations. Second, while there were several students who completed many goals over the weeks, other participants by the end had only completed one or two. No clear pattern could be detected through the completion of such a small number of goals. Third, there may have been fundamental reasons why participants could not complete certain goals (i.e. they were not able to prepare meals for their family because of household norms and rules). These issues may have led to an inaccurate measure of any relationship between the selection of family- or friend-based goals and perceived social or family norms. 
Although no conclusions could be reached in the current investigation, finding salient norms for this age group is an important area for future research into obesity prevention.

\section{Strengths and Limitations}

Pink and Dude Chefs, Phase 2 combined instruction of nutrition concepts with culinary skills in a safe, encouraging environment. Nutrition and culinary interventions that reconnect youth with where their food comes from are an important step towards reducing the rate of adolescent obesity (Nelson et al., 2013). Interventions that combine both knowledge and skills building increase the likelihood that a behavior change will occur. The program was based on behavioral change theories that have been previously shown to target constructs most likely to induce change, thus increasing the odds of program success. Several factors that have been shown to mediate healthful eating in adolescents were targeted and participants drew connections between dietary behaviors and positive health outcomes.

The Bright Futures after-school program at Mesa Middle School was an ideal platform for Pink and Dude Chefs, Phase 2. Because the program had been implemented at the site previously, there were preexisting relationships with staff members that facilitated implementation. In addition the program was already a popular afterschool choice in the student community after having been run there for several years. A wellstocked kitchen and classroom were made available for the program and the Bright Futures staff assisted as needed. Because incorporating nutrition/culinary education into the school day has many curricular time constraints and competes with mandatory educational content, the after-school program setting was ideal. Nelson et al. (2013) 
suggested that after-school are likely the best setting for in-depth education programs to help youths apply culinary concepts in experiential cooking classes.

The multicultural theme of Pink and Dude Chefs, Phase 2 was an excellent way to increase participants' international awareness and expose them to various food and eating cultures from around the globe. Participants drew connections between dietary habits and health outcomes for each topic country. Very few school-based culinary programs with a multicultural theme exist currently, but a UK-based after-school culinary program called Cooking Communities found significant improvements in meal preparation skills and cultural awareness in adolescents following a series of cooking classes focused on various cultural events (Gatenby et al., 2011). This approach has potential to become a valuable method for delivering culinary skills education. Furthermore, future Pink and Dude Chefs, Phase 2 program development might include a measure of cultural awareness.

Seventh and eighth grade students were an ideal target audience for this intervention because adolescence is a key time to establish healthy behaviors (Story et al., 2002). The group-work aspect allowed the participants to practice team-building skills and learn from their peers. Employers increasingly look for the soft skills of communication, team work and group problem solving in future employees (Andrews \& Higson, 2008). An unmeasured but important outcome of this program may be in acquisition and improvement in some of these soft skills. The teamwork aspect also forced each participant to be held accountable for their actions; for example, the students could not leave the kitchen until each small group had entirely cleaned their workstation and washed all of their dishes. 
Another positive aspect of the program design and staffing was that the participants seemed to deeply value the mentoring and attention from the college-aged program leaders and enjoyed building relationships throughout the session. A majority of the program leaders were Nutrition students at Cal Poly. Using Nutrition majors was highly beneficial because the leaders possessed the appropriate science-based knowledge of nutrition, food safety, and other relevant topics needed to address topics as discussions developed. Most of the program leaders enjoyed cooking themselves and therefore were able to offer tips and stories from their own experiences to the middle school students. Possibly through observation of college students demonstrating their passion for food and cooking, the participants developed a deeper admiration of these skills. Nelson et al. (2013) confirmed that observational learning raises self-efficacy by increasing the belief that one can complete the task equally well. Furthermore, program leaders may have served as positive role models for the participants. Previous studies have shown that a strong positive relationship exists between adolescents having an adult role model and reduction of risky behaviors, higher self-esteem, and higher grades (Beier, Rosenfeld, Spitalny, Zansky, \& Bontempo, 2000; Yancey, Siegel, \& McDaniel, 2002).

Pink and Dude Chefs, Phase 2 allowed for exposure to new foods and ingredients each week. For example, most of the participants had never tried quinoa before the program. Though skeptical at first, all participants loved this new ingredient after creating the Quinoa Black Bean Salad recipe. Participants also learned to draw connections between a prepared meal and the individual ingredients within it. For example, the participants were surprised to experience that an entire bag of fresh chopped spinach was added to the filling for the Stuffed Manicotti recipe. They were even more surprised to 
learn that once cooked, they could not even taste the spinach although the nutritional value of the dish was improved. This type of direct experience helps to dispel common myths such as healthy foods not tasting good (Nicklas et al., 2013). Another valuable skill that participants practiced was recipe substitution and flexibility. If a specific recipe ingredient was not available, a suitable replacement item was used instead. When participants learn to creatively work with what's available and seasonal, barriers to healthy eating such as affordability and accessibility are decreased.

Another strength was the family involvement aspect of Pink and Dude Chefs, Phase 2. At the beginning of each lesson, participants were encouraged to share their weekly goal sheet experiences with the class. A majority of them reported recreating the recipes at home for their families. The Family Fiesta celebration was not only an enjoyable experience for the participants and their loved ones; it was also an opportunity for the students to showcase their newly developed culinary skills. As stated previously, survey measures (i.e. perceived social and family norms) taken after the Family Fiesta celebration may be an area for future research. Most importantly, the participants seemed to thoroughly enjoy the Pink and Dude Chefs, Phase 2 program. They demonstrated excitement each week and felt a strong sense of pride in their recipe creations.

Study limitations should also be noted. Most importantly, the small sample size $(n=16)$ limited the statistical power to detect changes in some variables. The participants were self-selected through a convenience sample, since participation was limited to a small group of students enrolled in the Bright Futures after-school program. Furthermore, there was no control group used in this study and therefore the results may have been due to other environmental influences that occurred during this time. In addition participants 
were not randomized to any condition therefore the sample may have been biased. For example, participants who chose this afterschool program may have had prior interest or experience with cooking and not be representative of a more general group of middle school students. Randomization of participants into a treatment group and a control group would have eliminated this bias and allowed for better generalization of results to other populations. However, program results may be generalizable to $7^{\text {th }}$ and $8^{\text {th }}$ grade students from predominantly Hispanic and low SES communities in semi-rural, agricultural counties in California.

Another limitation was that the Program Coordinator was responsible for running the program, collecting data, performing statistical analysis, and analyzing results. This may have led to inflated outcome measures if the participants had a desire to please the Program Coordinator. Assigning a Site Manager or another research assistant to some of these roles could have eliminated this potential researcher bias. Additionally, the fact that the Program Coordinator running the program may influence the outcomes based on personal factors (i.e. teaching style, enthusiasm, knowledge and skill) limits the generalizability of the results. Lastly, it was assumed that the program was run the same during Fall 2013 and Winter 2014 and therefore the data was combined. However, there may have been unacknowledged differences that influenced the data.

Attendance was a significant limitation for this study. Although attendance for the Bright Futures program is generally high, there were several other programs within Bright Futures that conflicted with Pink and Dude Chefs. For example, about half of the participants missed lessons because they were also involved with drama class. Additionally, there were several participants who attended Pink and Dude Chefs regularly 
but missed the last lesson and so did not fill out a post-survey. These students were not counted in the final analysis sample. Results could have been analyzed by attendance, but this was not done due to the very small sample size. Results from Pink Chefs showed that although participants with high attendance demonstrated a greater in scores to knowledge-based questions, a statistically significant difference was not detected (Chessen, 2008). Attendance would likely be a problem for most after-school programs; therefore programmers should be prepared for attendance issues when working with after-school populations. 


\section{CHAPTER 5}

\section{Conclusions}

Due to the complex of interrelated causes of adolescent obesity, approaches to prevent and treat the problem must also be multifactorial in nature. There appears to be a relationship between the decline of culinary skills and poor dietary habits which contribute to childhood obesity (Nelson et al., 2013). Nutrition and culinary interventions based on behavioral change theories have been shown to be an effective way to improve mediators of healthy eating in adolescents, which, if causally associated, may lead to long-term health-promoting behaviors. It is crucial that such interventions address both knowledge- and skill-building for individuals to be able to enact dietary recommendations. Furthermore, these skills impact both individual and environmental determinants of behavior. Pink and Dude Chefs, Phase 2 significantly improved participants' nutrition knowledge and cooking skills.

A limited number of studies have assessed the impact of nutrition and culinary interventions on modifying obesity-related risk factors in adolescents. Although evidence does exist that supports their ability to improve mediators of healthful eating, the limitations are abundant. A majority of these studies did not have control groups, did not collect follow-up data, and did not include parents in the program (Nelson et al., 2013). Most importantly, it is difficult to reliably measure factors such as cooking skills and normative beliefs through surveys; therefore it is not fully understood how these factors translate into long-term behavioral changes.

Directions for future research include initiating large-scale randomized-controlled trials in nutrition and culinary education, improving the scales and survey questions 
currently used to measure mediators of healthy eating, and exploring the relationship between social and family normative beliefs and attitudes about cooking and eating healthfully in adolescents. Additionally, maximizing family involvement in nutrition and culinary interventions may lead to better outcomes. Another area of exploration for future research may be the psychological outcomes of adolescents from involvement in nutrition and culinary interventions. A possibility exists that teaching nutrition and cooking in a group setting using college-aged leaders may have a variety of unmeasured positive influences on the young teens' development.

There is a need for nutrition and culinary interventions in the United States, especially those which target high-risk populations such as Hispanic and low SES adolescents. Empowering youth by improving food-related knowledge and skill is an important step towards improving the health of our nation. 


\section{REFERENCES}

Alberga, A. S., Sigal, R. J., Goldfield, G., Prud'homme, D., \& Kenny, G. P. (2012). Overweight and obese teenagers: why is adolescence a critical period? Pediatric Obesity, 7(4), 261-73.

Allen, M. L., Elliott, M. N., Morales, L. S., Diamant, A. L., Hambarsoomian, K., \& Schuster, M. A. (2007). Adolescent participation in preventive health behaviors, physical activity, and nutrition: differences across immigrant generations for Asians and Latinos compared with Whites. American Journal of Public Health, 97(2), 33743.

Andrews, J., \& Higson, H. (2008). Graduate employability, 'soft skills' versus 'hard' business knowledge: A European study. Higher Education in Europe, 33(4), 411-22.

Arcan, C., Larson, N., Bauer, K., Berge, J., Story, M., \& Neumark-Sztainer, D. (2014). Dietary and weight-related behaviors and body mass index among Hispanic, Hmong, Somali, and White adolescents. Journal of the Academy of Nutrition and Dietetics, 114(3), 375-83.

Ball, K., Jeffery, R. W., Abbott, G., McNaughton, S. A., \& Crawford, D. (2010). Is healthy behavior contagious: Associations of social norms with physical activity and healthy eating. The International Journal of Behavioral Nutrition and Physical Activity, 7(1), 86.

Baranowski, T., Davis, M., Resnicow, K., Baranowski, J., Doyle, C., Lin, L. S., et al. (2000). Gimme 5 fruit, juice, and vegetables for fun and health: Outcome evaluation. Health Education \& Behavior, 27(1), 96-111.

Beier, S. R., Rosenfeld, W. D., Spitalny, K. C., Zansky, S. M., \& Bontempo, A. N. (2000). The potential role of an adult mentor in influencing high-risk behaviors in adolescents. Archives of Pediatrics \& Adolescent Medicine, 154(4), 327.

Branscum, P., \& Sharma, M. (2012). After-school based obesity prevention interventions: a comprehensive review of the literature. International Journal of Environmental Research and Public Health, 9(4), 1438-57.

Brown, B. J., \& Hermann, J. R. (2005). Cooking classes increase fruit and vegetable intake and food safety behaviors in youth and adults. Journal of Nutrition Education and Behavior, 37(2), 104-05.

Butte, N. F., Cai, G., Cole, S. A., \& Comuzzie, A. G. (2006). Viva la Familia Study: Genetic and environmental contributions to childhood obesity and its comorbidities in the Hispanic population. The American Journal of Clinical Nutrition, 84(3), 64654. 
California Department of Education. (2014). Student poverty: FRPM data. Retrieved from http://www.cde.ca.gov/ds/sd/sd/filessp.asp

California Department of Public Health: The Network for a Healthy California. (2012). Compendium of surveys for nutrition education obesity prevention. Retrieved from http://www.cdph.ca.gov/programs/cpns/Documents/Compendium\%20of\%20Surveys .pdf

Cawley, J., \& Meyerhoefer, C. (2012). The medical care costs of obesity: An instrumental variables approach. Journal of Health Economics, 31(1), 219-30.

Centers for Disease Control and Prevention. (2014). Adolescent and school health: Childhood obesity facts. Retrieved January 20, 2014, from http://www.cdc.gov/healthyyouth/obesity/facts.htm

Centers for Disease Control and Prevention. (2012a). Overweight and obesity: Basics about childhood obesity. Retrieved January 20, 2014, from http://www.cdc.gov/obesity/childhood/basics.html

Centers for Disease Control and Prevention. (2012b). Overweight and obesity: What causes overweight and obesity? Retrieved January 20, 2014, from http://www.cdc.gov/obesity/adult/causes/index.html

Chessen, J. A. (2008). The development and pilot of a culinary intervention designed using the social cognitive theory to teach nutrition to adolescent girls. San Luis Obispo, California: California Polytechnic State University.

Christakis, N. A., \& Fowler, J. H. (2007). The spread of obesity in a large social network over 32 years. The New England Journal of Medicine, 357, 370-79.

Condrasky, M. D., \& Hegler, M. (2010). How culinary nutrition can save the health of a nation. Journal of Extension, 48(2), 1-6.

Condrasky, M., Parisi, M., Wall-Bassett, B., \& Warmin, A. (2008). Process evaluation of the "Cooking with a CHEF" culinary nutrition education program. Journal of Nutrition Education and Behavior, 40(4), S73.

Condrasky, M. D., Williams, J. E., Catalano, P. M., \& Griffin, S. F. (2011). Development of psychosocial scales for evaluating the impact of a culinary nutrition education program on cooking and healthful eating. Journal of Nutrition Education and Behavior, 43(6), 511-6.

Cunningham, S.A., Kramer, M.R., Venkat Narayan, K. M. (2014). Incidence of childhood obesity in the United States. The New England Journal of Medicine, 370, 403-411. 
Cunningham-Sabo, L., \& Lohse, B. (2014). Impact of a school-based cooking curriculum for fourth-grade students on attitudes and behaviors is influenced by gender and prior cooking experience. Journal of Nutrition Education and Behavior, 46(2), 11020.

Davis, J. N., Ventura, E. E., Cook, L. T., Gyllenhammer, L. E., \& Gatto, N. M. (2011). LA Sprouts: A gardening, nutrition, and cooking intervention for Latino youth improves diet and reduces obesity. Journal of the American Dietetic Association, $111(8), 1224-30$.

Dougherty, K., \& Silver, C. (2007). Chef-nutritionist teams spark enjoyment and learning in cooking education series for 8- to 12-year-olds. Journal of Nutrition Education and Behavior, 39(4), 237-38.

Fitzgerald, A., Heary, C., Kelly, C., Nixon, E., \& Shevlin, M. (2013). Self-efficacy for healthy eating and peer support for unhealthy eating are associated with adolescents' food intake patterns. Appetite, 63, 48-58.

Freedman, D. S., Khan, L. K., Serdula, M. K., Dietz, W. H., Srinivasan, S. R., \& Berenson, G. S. (2005). The relation of childhood BMI to adult adiposity: The Bogalusa Heart Study. Pediatrics, 115(1), 22-7.

Freedman, D. S., Mei, Z., Srinivasan, S. R., Berenson, G. S., \& Dietz, W. H. (2007). Cardiovascular risk factors and excess adiposity among overweight children and adolescents: The Bogalusa Heart Study. The Journal of Pediatrics, 150(1), 12-17.

Gatenby, L. A., Donnelly, J., \& Connell, R. (2011). Cooking Communities: Using multicultural after-school cooking clubs to enhance community cohesion. Nutrition Bulletin, 36(1), 108-112.

Glanz, K., Rimer B. K., \& Viswanath. K. (2008). Health behavior and health education: Theory, research, and practice ( $4^{\text {th }}$ ed.). San Francisco, CA: Jossey-Bass.

Halford, J., Caraher, M., Seeley, A., Wu, M., \& Lloyd, S. (2013). When chefs adopt a school? An evaluation of a cooking intervention in English primary schools. Appetite, 62, 50-59.

Hartmann, C., Dohle, S., \& Siegrist, M. (2013). Importance of cooking skills for balanced food choices. Appetite, 65, 125-31.

HealthyPeople.gov. (2014). 2020 topics and objectives: Nutrition and weight status. Retrieved from http://www.healthypeople.gov/2020/topicsobjectives2020/objectiveslist.aspx?topicI $\mathrm{d}=29$ 
Jacobson, D., \& Melnyk, B. M. (2011). Psychosocial correlates of healthy beliefs, choices, and behaviors in overweight and obese school-age children: A primary care healthy choices intervention pilot study. Journal of Pediatric Nursing, 26(5), 45664.

Keihner, A. J., Meigs, R., Sugerman, S., Backman, D., Garbolino, T., \& Mitchell, P. (2011). The Power Play! Campaign's School Idea \& Resource Kits improve determinants of fruit and vegetable intake and physical activity among fourth- and fifth-grade children. Journal of Nutrition Education and Behavior, 43(4 Suppl 2), S122-9.

Krebs-Smith, S. M., Guenther, P. M., Subar, A. F., Kirkpatrick, S. I., \& Dodd, K. W. (2010). Americans do not meet federal dietary recommendations. The Journal of Nutrition, 140(10), 1832-8.

Kushi, L. H., Byers, T., Doyle, C., Bandera, E. V., McCullough, M., Gansler, T., et al. (2006). American Cancer Society guidelines on nutrition and physical activity for cancer prevention: Reducing the risk of cancer with healthy food choices and physical activity. CA: A Cancer Journal for Clinicians 2006(56), 254-81.

Larson, N. I., Perry, C. L., Story, M., \& Neumark-Sztainer, D. (2006). Food preparation by young adults is associated with better diet quality. Journal of the American Dietetic Association, 106(12), 2001-07.

Larson, N. I., Story, M., Eisenberg, M. E., \& Neumark-Sztainer, D. (2006). Food preparation and purchasing roles among adolescents: Associations with sociodemographic characteristics and diet quality. Journal of the American Dietetic Association, 106(2), 211-18.

Laska, M. N., Larson, N. I., Neumark-Sztainer, D., \& Story, M. (2012). Does involvement in food preparation track from adolescence to young adulthood and is it associated with better dietary quality? Findings from a 10-year longitudinal study. Public Health Nutrition, 15(7), 1150-58.

Levy, J., \& Auld, G. (2004). Cooking classes outperform cooking demonstrations for college sophomores. Journal of Nutrition Education and Behavior, 36(4), 197-203.

Li, C., Ford, E. S., Zhao, G., \& Mokdad, A. H. (2009). Prevalence of pre-diabetes and its association with clustering of cardiometabolic risk factors and hyperinsulinemia among US adolescents: NHANES 2005-2006. Diabetes Care, 32(2), 342-47.

Liquori, T., Koch, P. D., Ruth Contento, I., \& Castle, J. (1998). The Cookshop Program: Outcome evaluation of a nutrition education program linking lunchroom food experiences with classroom cooking experiences. Journal of Nutrition Education, 30(5), 302-13. 
Lukas, C. V, \& Cunningham-Sabo, L. (2011). Qualitative investigation of the Cooking with Kids program: Focus group interviews with fourth-grade students, teachers, and food educators. Journal of Nutrition Education and Behavior, 43(6), 517-24.

McClain, A. D., Chappuis, C., Nguyen-Rodriguez, S. T., Yaroch, A. L., \& Spruijt-Metz, D. (2009). Psychosocial correlates of eating behavior in children and adolescents: A review. The International Journal of Behavioral Nutrition and Physical Activity, 6, 54.

Morland, K., Wing, S., \& Diez Roux, A. (2002). The contextual effect of the local food environment on residents' diets: The atherosclerosis risk in communities study. American Journal of Public Health, 92, 1761-67.

National Institutes of Health: National Heart, Lung and Blood Institute. (2012). Disease and conditions index: What are overweight and obesity? Retrieved January 20, 2014, from http://www.nhlbi.nih.gov/health/health-topics/topics/obe/

Nelson, S. A., Corbin, M. A., \& Nickols-Richardson, S. M. (2013). A call for culinary skills education in childhood obesity-prevention interventions: Current status and peer influences. Journal of the Academy of Nutrition and Dietetics, 113(8), 103136.

Nicklas, T. A., Baranowski, T., Baranowski, J. C., (2013). Family and child-care provider influences on preschool children's fruit, juice, and vegetable consumption. Nutrition Reviews, 59(7), 224-35.

Ogden, C. L., Carroll, M. D., Kit, B. K., \& Flegal, K. M. (2014). Prevalence of childhood and adult obesity in the United States, 2011-2012. JAMA: The Journal of the American Medical Association, 311(8), 806-14.

Ogden, C. L., Lamb, M. M., Carroll, M. D., Flegal, K. M. (2010). Obesity and socioeconomic status in children: United States 1988-1994 and 2005-2008. National Center for Health Statistics Data Brief no. 51. Retrieved from http://www.cdc.gov/nchs/data/databriefs/db51.pdf

Opalinski, A. (2010). What are key factors in Haitian and Hispanic children and obesity: Parent answers. The Journal for Nurse Practitioners : JNP, 6(4), 281-286.

Oude Luttikhuis, H., Baur, L., Jansen, H., Shrewsbury, V. A., O’Malley, C., Stolk, R. P., et al. (2009). Interventions for treating obesity in children. The Cochrane Database of Systematic Reviews 2009, (1), CD001872.

Plantinga, A. J., \& Bernell, S. (2007). The association between urban sprawl and obesity: Is it a two-way street? Journal of Regional Science, 47, 857-879. 
Popkin, B. M. (1993). Nutritional patterns and transitions. Population and Development Review, 19, 138-57.

Pulgarón, E. R. (2013). Childhood obesity: A review of increased risk for physical and psychological comorbidities. Clinical Therapeutics, 35(1), A18-32.

Rabiei, L., Sharifirad, G. R., Azadbakht, L., \& Hassanzadeh, A. (2013). Understanding the relationship between nutritional knowledge, self-efficacy, and self-concept of high-school students suffering from overweight. Journal of Education and Health Promotion, 2, 39.

Resnicow, K., Davis-Hearn, M., Smith, M., Baranowski, T., Lin, L. S., Baranowski, J., et al. (1997). Social-cognitive predictors of fruit and vegetable intake in children. Health Psychology, 16(3), 272-76.

Salvy, S. J., de la Haye, K., Bowker, J. C., \& Hermans, R. C. J. (2012). Influence of peers and friends on children's and adolescents' eating and activity behaviors. Physiology and Behavior, 106(3), 369-78.

Sheehan, T. (2013). Pink and Dude Chefs: A nutrition and culinary intervention for middle school students. San Luis Obispo, California: California Polytechnic State University.

Singh, G. K., Kogan, M. D., Van Dyck, P. C., \& Siahpush, M. (2008). Racial/ethnic, socioeconomic, and behavioral determinants of childhood and adolescent obesity in the United States: Analyzing independent and joint associations. Annals of Epidemiology, 18(9), 682-95.

Skelton, J. A., DeMattia, L., Miller, L., \& Olivier, M. (2006). Obesity and its therapy: From genes to community action. Pediatric Clinics of North America, 53(4), 77794.

Story, M., Neumark-Sztainer, D., \& French, S. (2002). Individual and environmental influences on adolescent eating behaviors. Journal of the American Dietetic Association, 102(3), S40-S51.

Stevenson, C., Doherty, G., Barnett, J., Muldoon, O. T., \& Trew, K. (2007). Adolescents' views of food and eating: Identifying barriers to healthy eating. Journal of Adolescence, 30(3), 417-34.

Townsend, N., \& Scriven, A. (2014). Public health mini-guides: Obesity. London, England: Churchill Livingstone.

Turner, G. (1999). Peer support and young people's health. Journal of Adolescence, 22, $567-572$. 
United States Census Bureau. (2014). State and county quick facts: Arroyo Grande California. Retrieved from http://quickfacts.census.gov/qfd/states/06/0602868.html

United States Department of Agriculture: Agricultural Marketing Service. (2014). Food deserts. Retrieved from https://apps.ams.usda.gov/fooddeserts/foodDeserts.aspx/

United States Department of Agriculture: ChooseMyPlate.gov. (2014). 10 tips nutrition education series. Retrieved from http://www.choosemyplate.gov/healthy-eatingtips/ten-tips.html

Wall, D. E., Least, C., Gromis, J., \& Lohse, B. (2012). Nutrition education intervention improves vegetable-related attitude, self-efficacy, preference, and knowledge of fourth-grade students. The Journal of School Health, 82(1), 37-43.

Winter, Y., Sankowski, R., \& Back, T. (2013). Genetic determinants of obesity and related vascular diseases. Vitamins and Hormones, 91, 29-48.

World Health Organization. (2014). Global strategy on diet, physical activity, and health: Child overweight and obesity. Retrieved from http://www.who.int/dietphysicalactivity/childhood/en/

Wrieden, W. L., Anderson, A. S., Longbottom, P. J., Valentine, K., Stead, M., Caraher, M., et al. (2007). The impact of a community-based food skills intervention on cooking confidence, food preparation methods and dietary choices - An exploratory trial. Public Health Nutrition, 10(2), 203-11.

Yancey, A. K., Siegel, J. M., \& McDaniel, K. L. (2002). Role models, ethnic identity, and health-risk behaviors in urban adolescents. Archives of Pediatrics \& Adolescent Medicine, 156(1), 55.

Zeinstra, G. G., Koelen, M. A., Kok, F. J., \& de Graaf, C. (2007). Cognitive development and children's perceptions of fruit and vegetables; A qualitative study. The International Journal of Behavioral Nutrition and Physical Activity, 4, 30. 


\section{APPENDICES}

\section{Appendix A: Pre- and Post-Survey}

\section{PINK AND DUDE CHEFS PHASE 2- AROUND THE WORLD Before we get started}

Instructions: We would like you to complete this survey. You may skip questions you do not want to answer but we hope that you will answer all of them. Any information about who you are will be kept secret. We will use a number on each survey instead of using your name. These numbers will not be connected to you in any way and will only be used for reports.

Part 1: Detalls about yourself The first few questions are about you. Please circle your answer.

1. What grade are you in?
a. 6th grade
b. 7 th grade
c. Bth grade

2. How old are you?

3. Are you a boy or a girl?
a. Boy
b. Girl

4. How many people live in your household (including yourself)?
a. 2
b. 3
c. 4
d. 5 or more

5. How would you describe yourself? (Circle ALL that apply to you)
a. American Indian or Alaska Native
b. Asian
c. Black or African American
d. Hispanic or Latino Mexican
e. Native Hawaiian or Other Pacific Islander
f. White/Caucasian
g. Other (

6. Have you participated in a cooking class before?
a. Yes
b. No

7. If so, what was it called?

a. 
Part 2: Cooking Skills How do YOU prepare meals and snacks? Please circle your answer.

1. In a normal week, what kinds of cooking did you do at home? (Circle ALL that apply)

a. Cook convenience foods and ready-made meals from a package

b. Put together ready-made ingredients to make a complete meal

c. Prepare dishes from basic ingredients, from scratch

d. Don't cook at all

e. Other, please explain

2. In a normal week, how often do you prepare and cook a meal from basic ingredients, from scratch? For example, making a meat and vegetable stir fry starting with raw meat and fresh vegetables. (Please circle only ONE)
a. Every day
b. Most days
c. Few days
d. Never

3. In a normal week: (Check ALL that apply)
a. I make meals for myself
b. I make snacks for myself
c. I make meals for others in my family
d. I make snacks for others in my family
e. I did NOT make any meals or snacks for myself or for my family

4. How important is it to learn to cook for YOURSELF?
a. Extremely important
b. Somewhat important
c. Important
d. Not very important
e. Not at all important

5. How important is it to learn to cook for your FAMILY?
a. Extremely important
b. Somewhat important
c. Important
d. Not very important
e. Not at all important

6. How important is it to learn to cook for your FRIENDS?
a. Extremely important
b. Somewhat important
c. Important 
d. Not very important

e. Not at all important

7. How confident are you that you can cook from basic ingredients, from scratch?

a. Extremely confident

b. Somewhat confident

c. Confident

d. Not very confident

e. Not at all confident

8. How confident are you that you can create meals using new foods and ingredients?
a. Extremely confident
b. Somewhat confident
c. Confident
d. Not very confident
e. Not at all confident

9. How confident are you that you can follow a simple recipe when cooking?
a. Extremely confident
b. Somewhat confident
c. Confident
d. Not very confident
e. Not at all confident

10. How confident are you that you can safely use a knife when cooking?
a. Extremely confident
b. Somewhat confident
c. Confident
d. Not very confident
e. Not at all confident 
Part 3: MyPlate We want you to tell us what you know about heaithful eating. Please fil in the bianks or circle your response.

1. Please fill in the names of the $\mathbf{5}$ food groups found on the USDA MyPlate. Just leave the line blank if you do not know the name of the group.

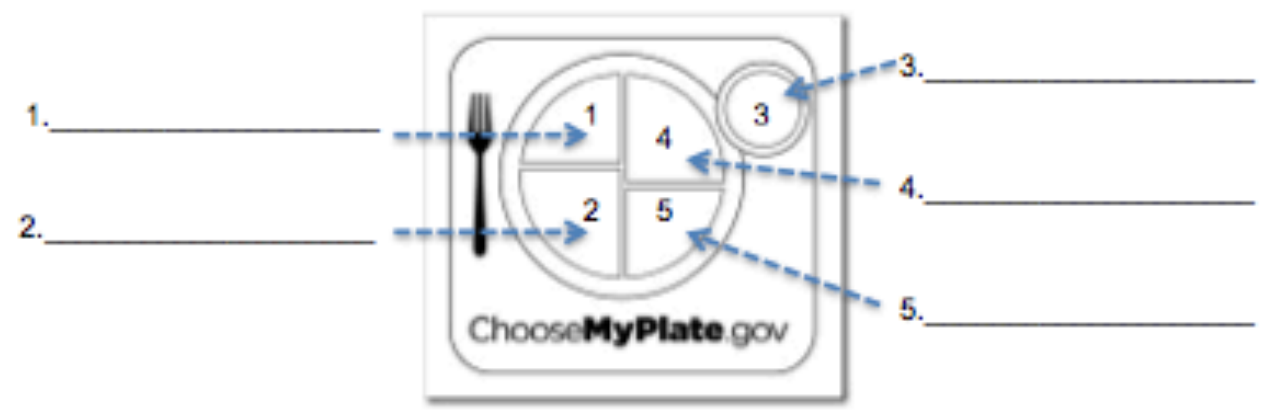

For questions 2 through 4, please circle either True or False for each statement.

2. Half of the grains I eat should be whole grains.

a. True

b. False

3. When eating a balanced meal, half of the food on my plate should be fruits \& vegetables.

a. True

b. False

4. Eating too fast may lead to eating too much food.

a. True

b. False

5. Eating while watching TV may lead to eating too much food.

a. True

b. False

6. Which of the following foods are good sources of protein? (Please circle ALL that apply)
a. Meat
f. Nuts \& seeds
b. Vegetables
g. Seafood
c. Eggs
h. Dairy products
d. Fruits
i. Tofu
e. Beans $\&$ peas 
Part 4: Family and Friends Since family and friends may affect your eating habits, we'd like to know more about them.

1. I think cooking for my FAMILY is fun and cool.
a. Agree strongly
b. Agree
c. Disagree
d. Strongly disagree

2. What my FAMILY thinks about cooking is important to me.
a. Agree strongly
b. Agree
c. Disagree
d. Strongly disagree

3. My FRIENDS think cooking is fun and cool.
a. Agree strongly
b. Agree
c. Disagree
d. Strongly disagree

4. What my FRIENDS think about cooking is important to me.
a. Agree strongly
b. Agree
c. Disagree
d. Strongly disagree

5. Most people in my FAMILY think that eating $3-4$ cups of fruits and vegetables each day is:
a. A very good thing
b. A good thing
c. Not important
d. I don't know

6. Most KIDS MY AGE think that eating 3-4 cups of fruits and vegetables each day is:
a. A very good thing
b. A good thing
c. Not important
d. I don't know 
Please circle the answer that best describes how much you agree ar disagree with each sentence beiow.

7. Many of my FRIENDS care about eating healthy food.
a. Agree strongly
b. Agree
c. Disagree
d. Strongly disagree

8. Many of my FRIENDS care about staying fit and exercising.
a. Agree strongly
b. Agree
c. Disagree
d. Strongly disagree

9. Most people in my FAMILY care about eating healthy food.
a. Agree strongly
b. Agree
c. Disagree
d. Strongly disagree

10. Most people in FAMILY care about staying fit and exercising.
a. Agree strongly
b. Agree
c. Disagree
d. Strongly disagree

Part 4: Fruits \& Vegetables We want to know what you think will happen if you eat fruits and vegetables every day. There are no right or wrong answers, just your opinion. Please circle the answer that best describes how much you agree or disagree with each sentence below.

If I eat fruits and vegetables every day

1. I will become stronger.
a. I agree very much
b. I agree a little
c. I am not sure
d. I disagree a little
e. I disagree very much

2. My friends will start eating them too.
a. I agree very much
b. I agree a little
c. I am not sure 

d. I disagree a liftle
e. I disagree very much

3. I will have more energy.
a. I agree very much
b. I agree a little
c. I am not sure
d. I disagree a little
e. I disagree very much

4. I will be healthier.
a. I agree very much
b. I agree a little
c. I am not sure
d. I disagree a little
e. I disagree very much

5. My family will be proud of me.
a. I agree very much
b. I agree a little
c. I am not sure
d. I disagree a little
e. I disagree very much

6. I will think better in class.
a. I agree very much
b. I agree a little
c. I am not sure
d. I disagree a little
e. I disagree very much

7. I will have a nicer smile and clearer skin.
a. I agree very much
b. I agree a little
c. I am not sure
d. I disagree a little
e. I disagree very much

Thanks for your help!!

Your time and opinions are very valuable to us!

\section{We look forward to having a GREAT Pink and Dude Chefs series and we can't wait to get} started cooking some DELICIOUS recipes! 


\section{Appendix B: Goal Sheets}

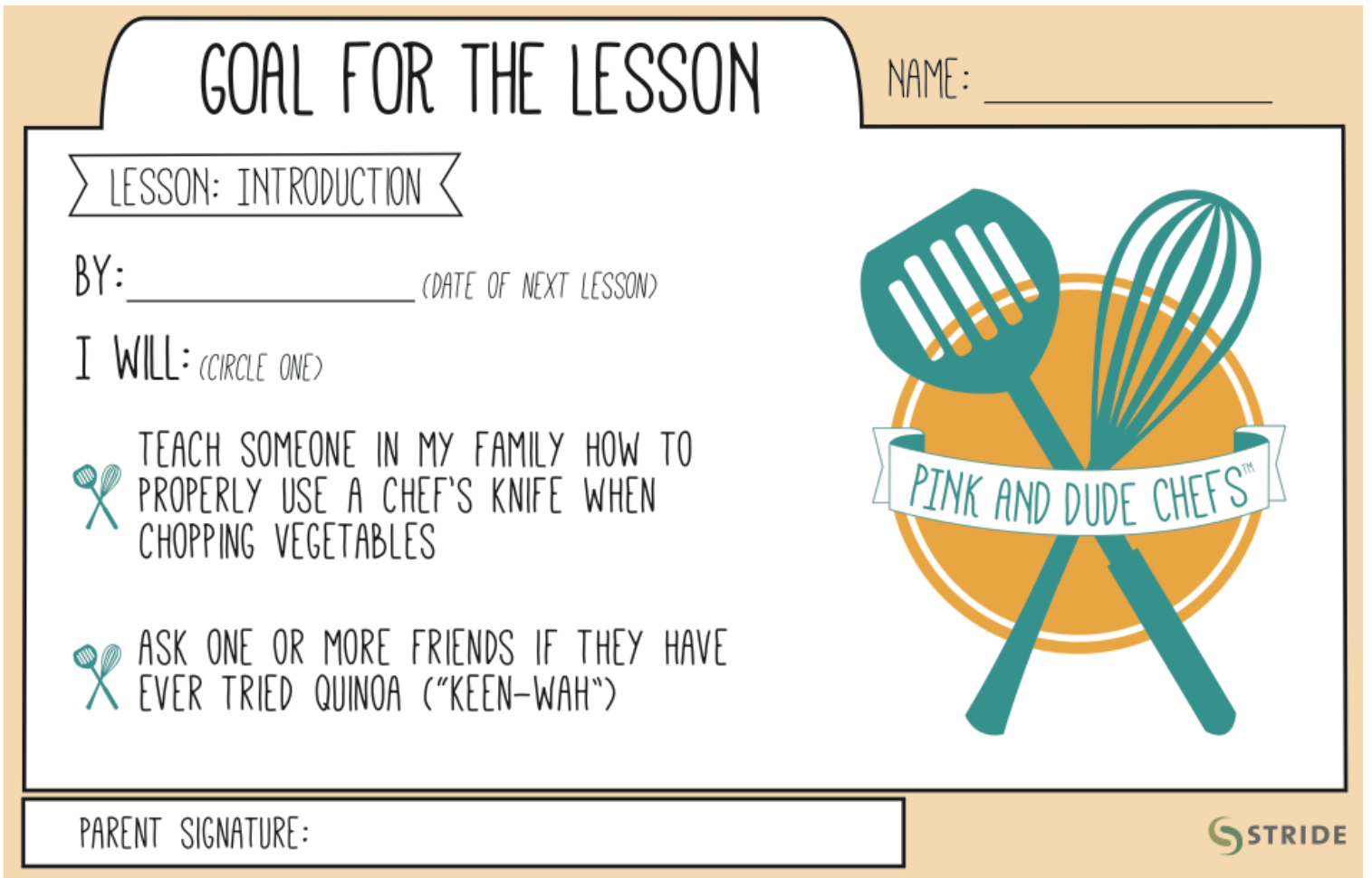

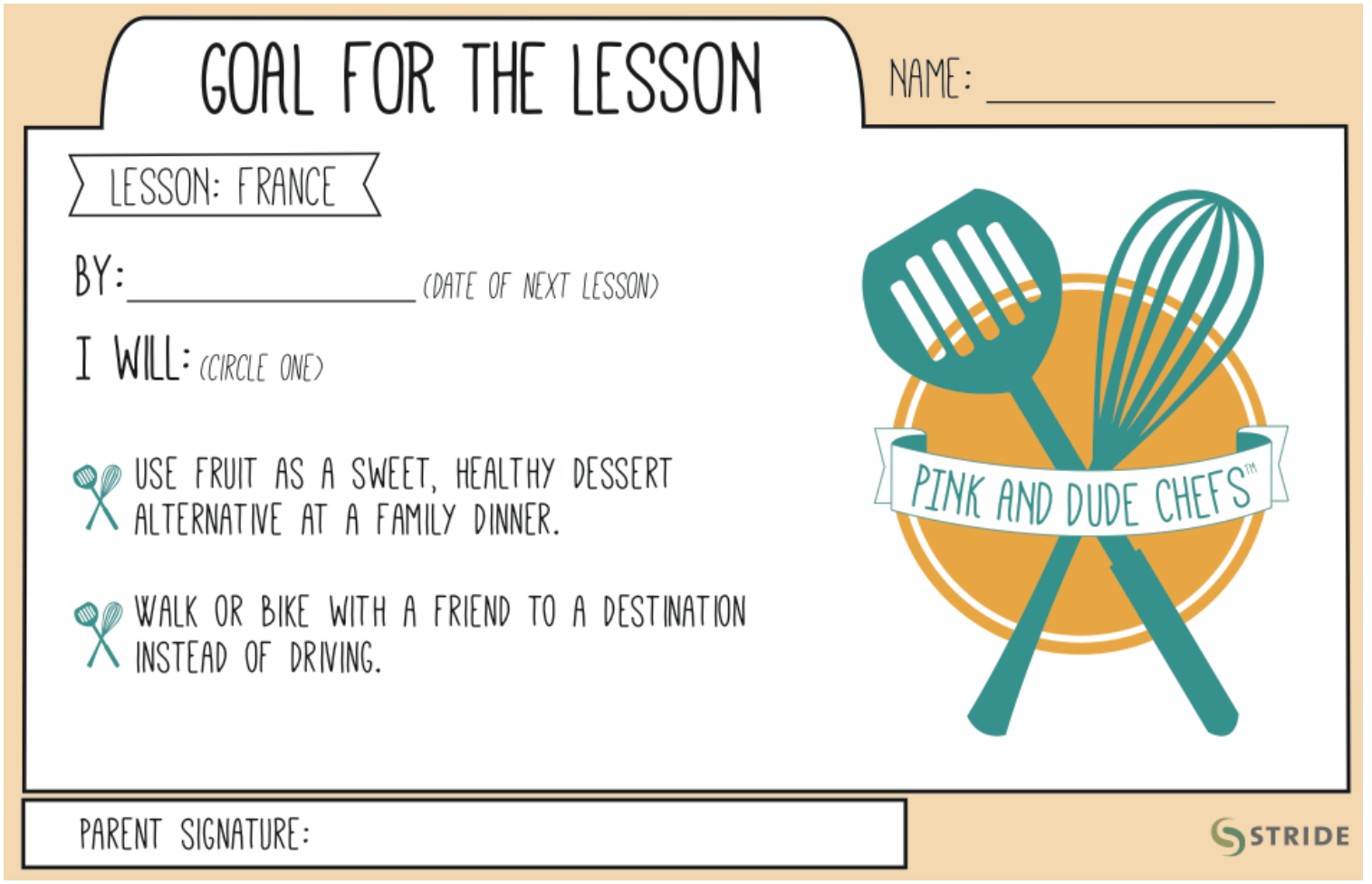



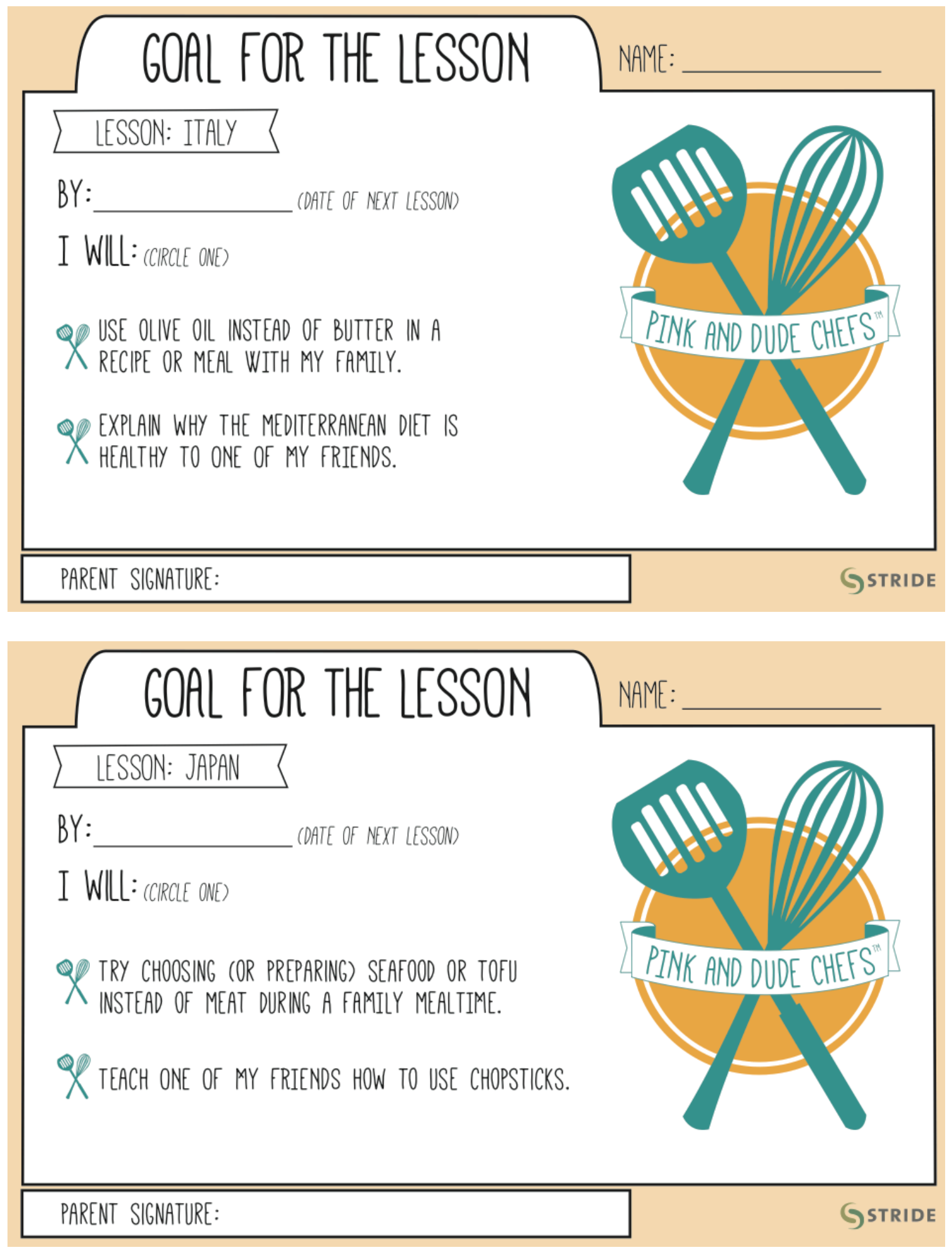


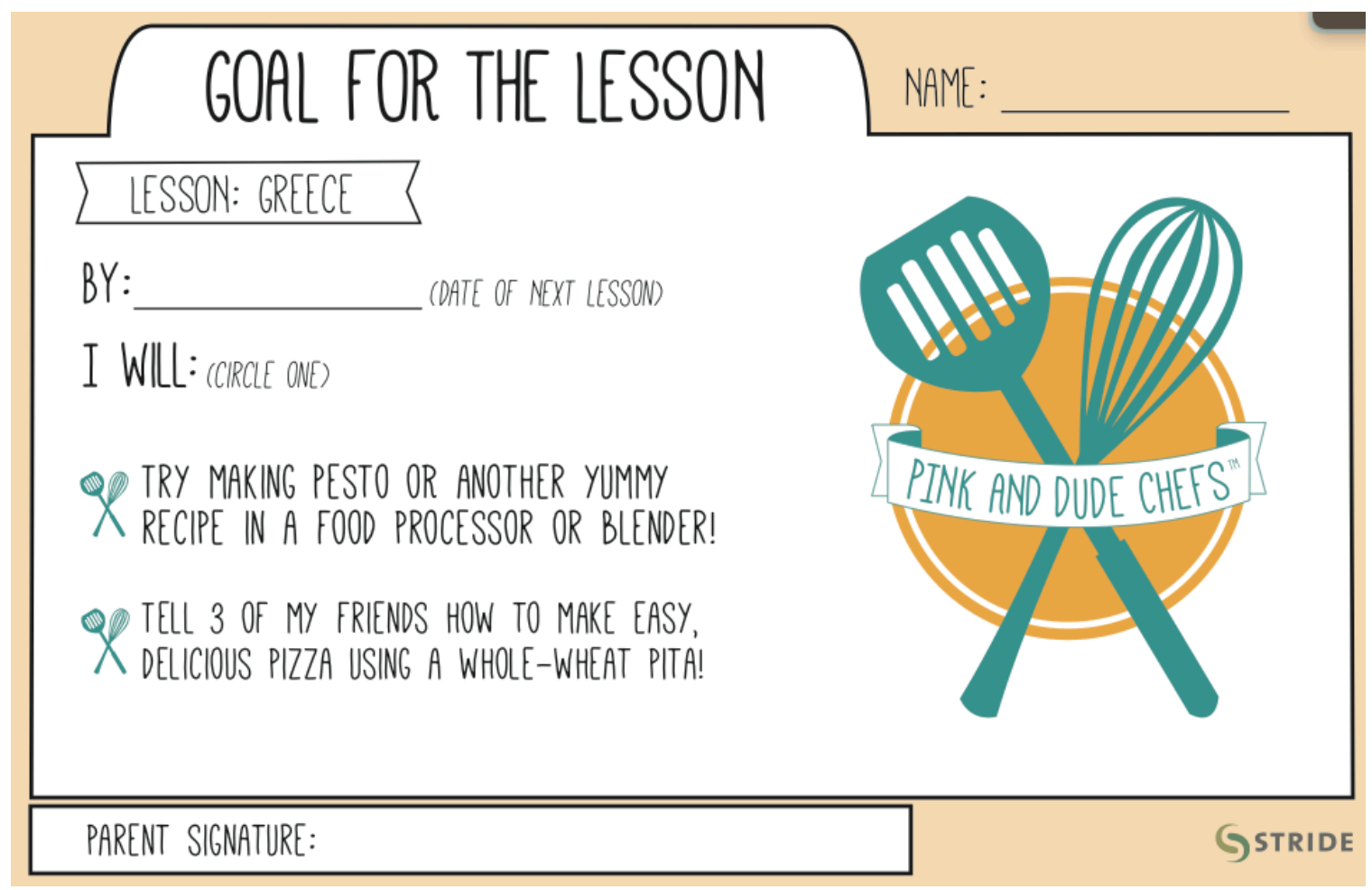

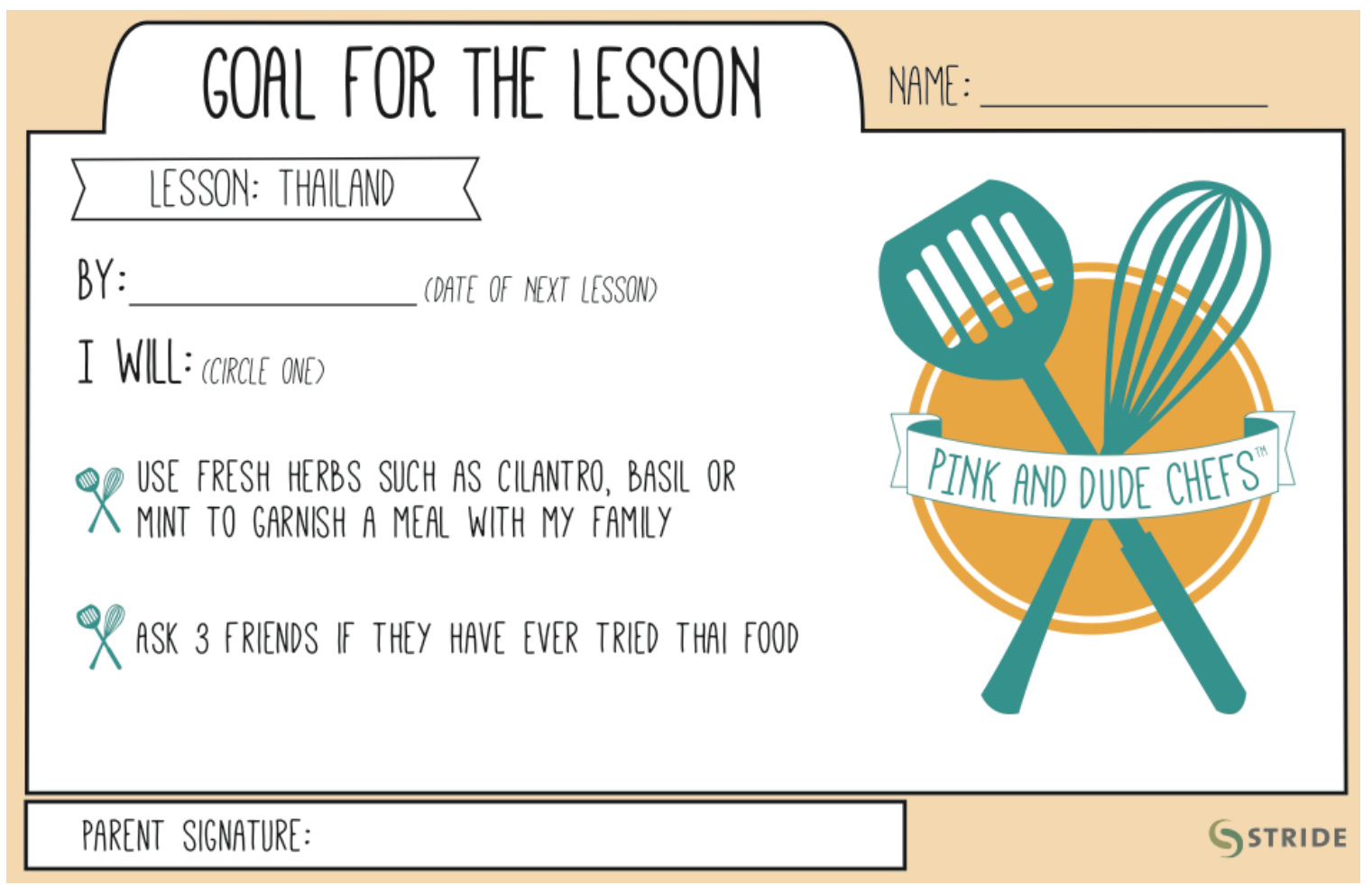




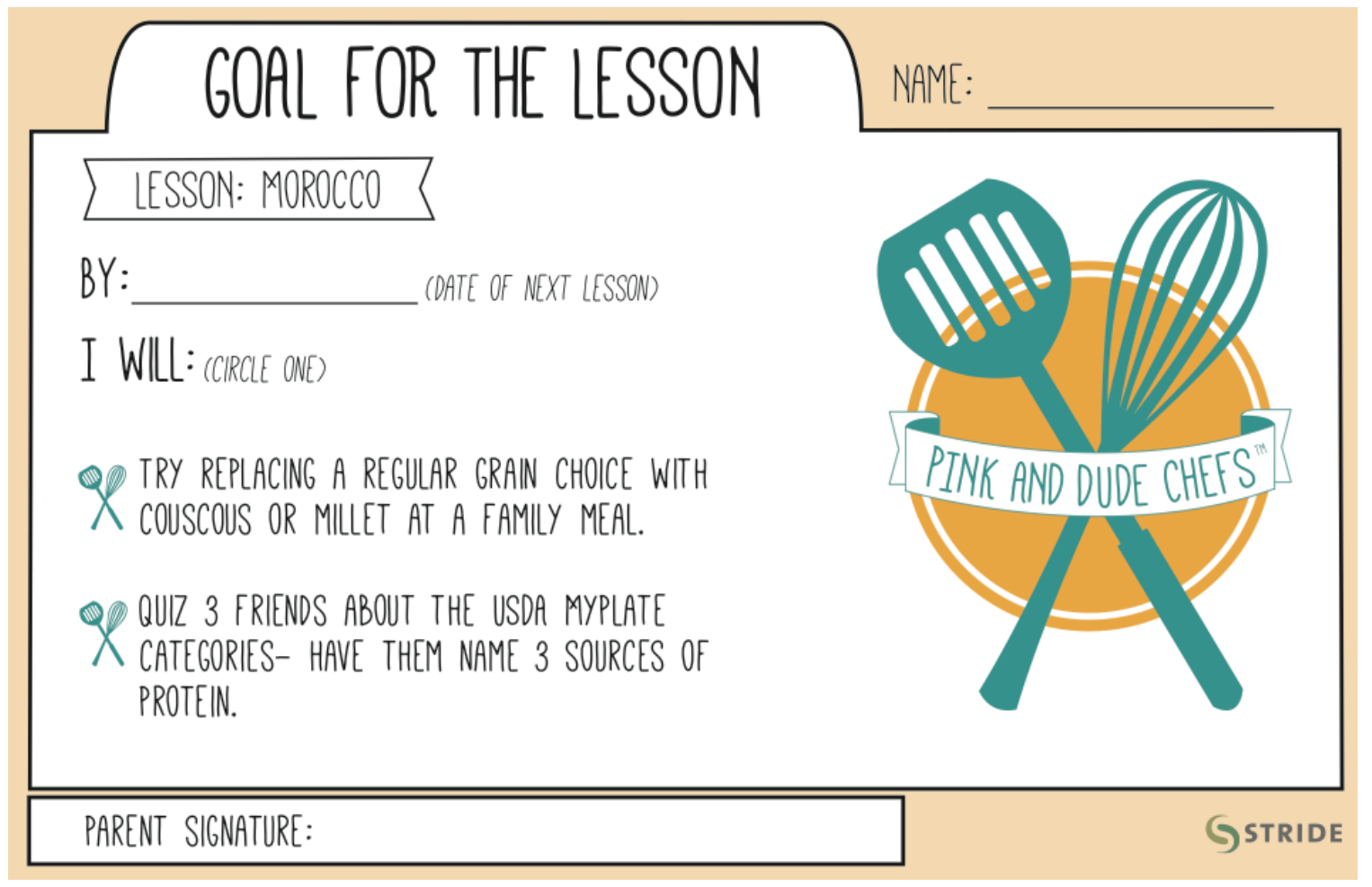




\section{Appendix C: Recipes}

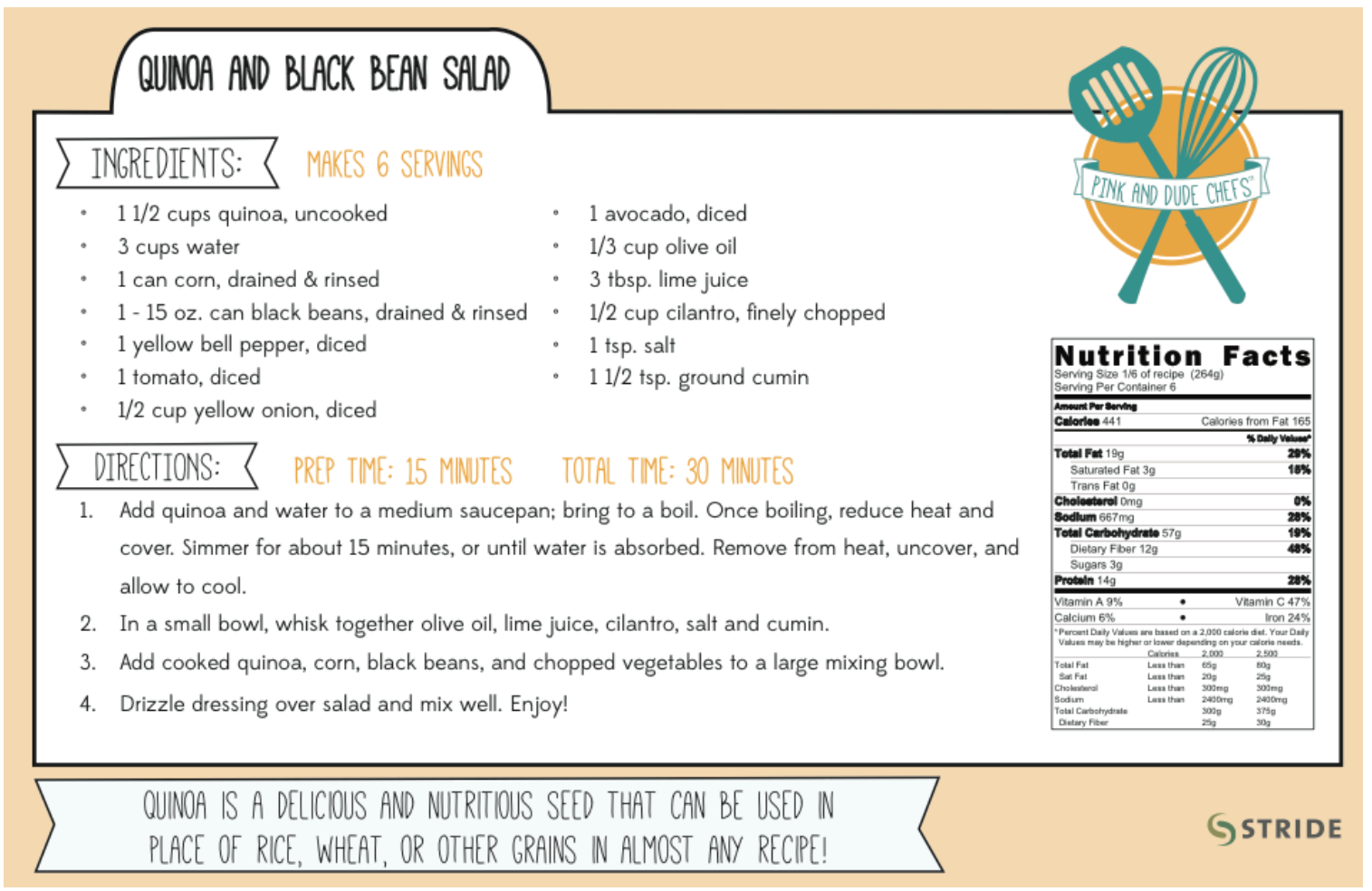

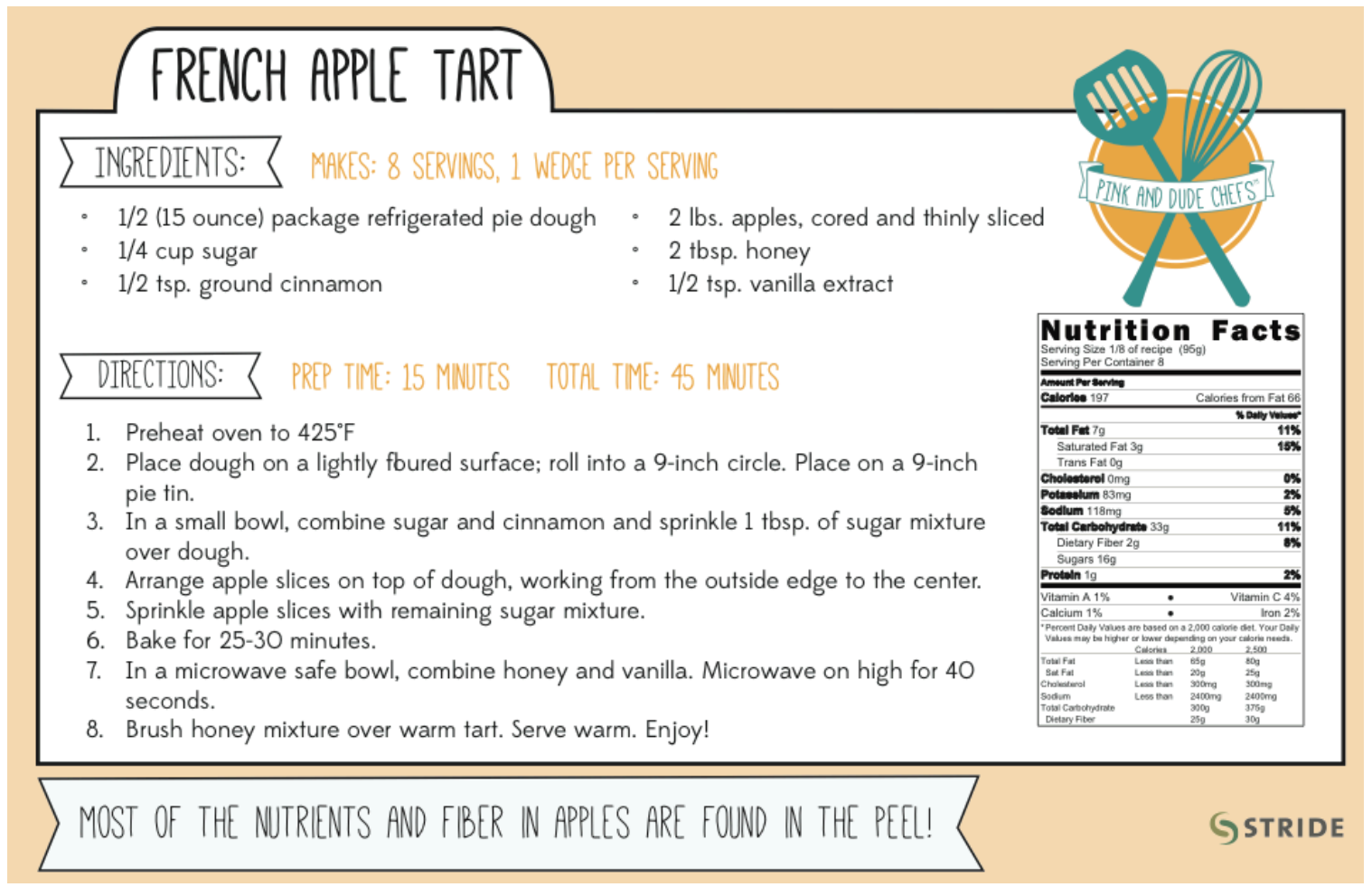




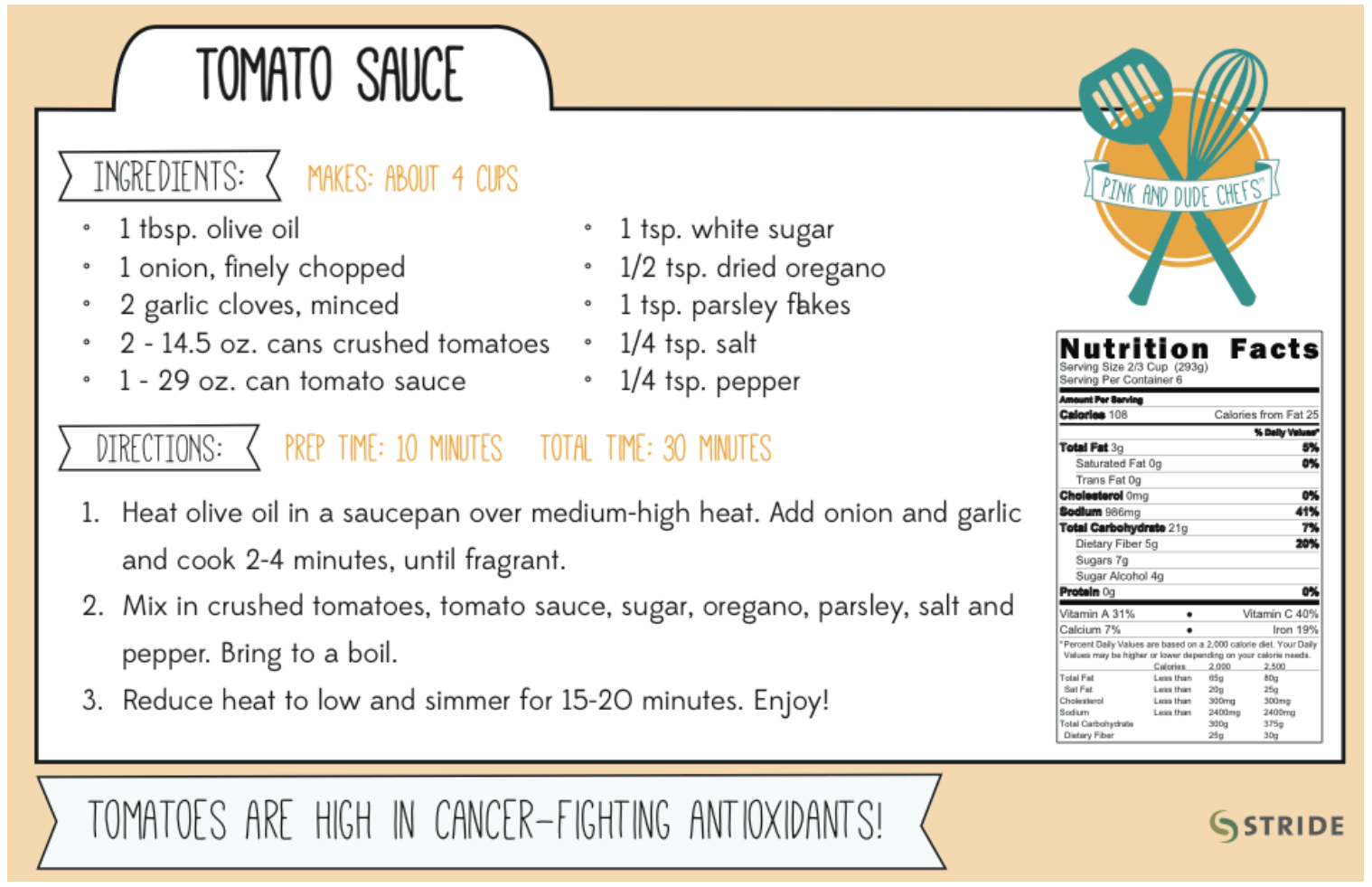

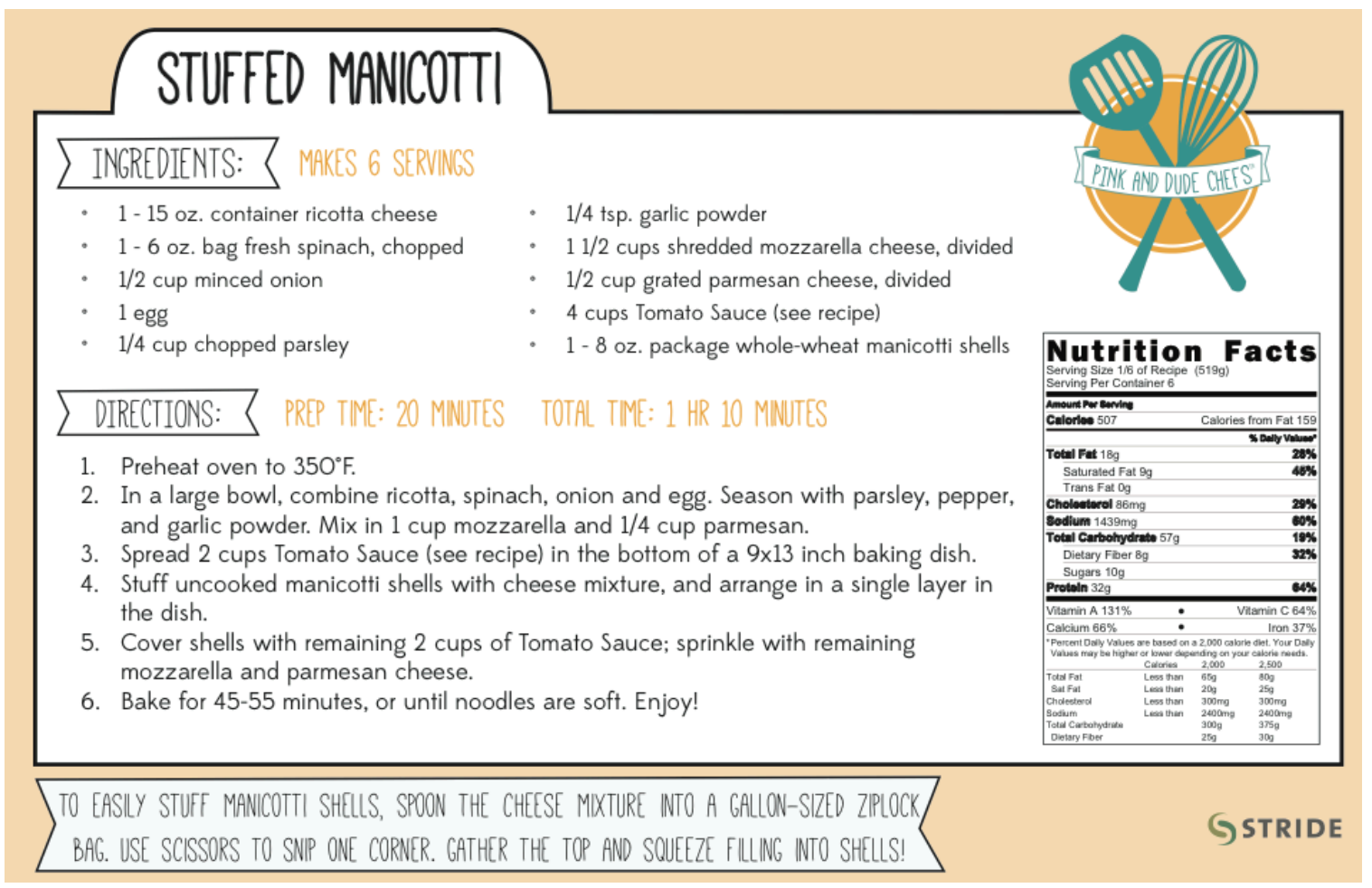



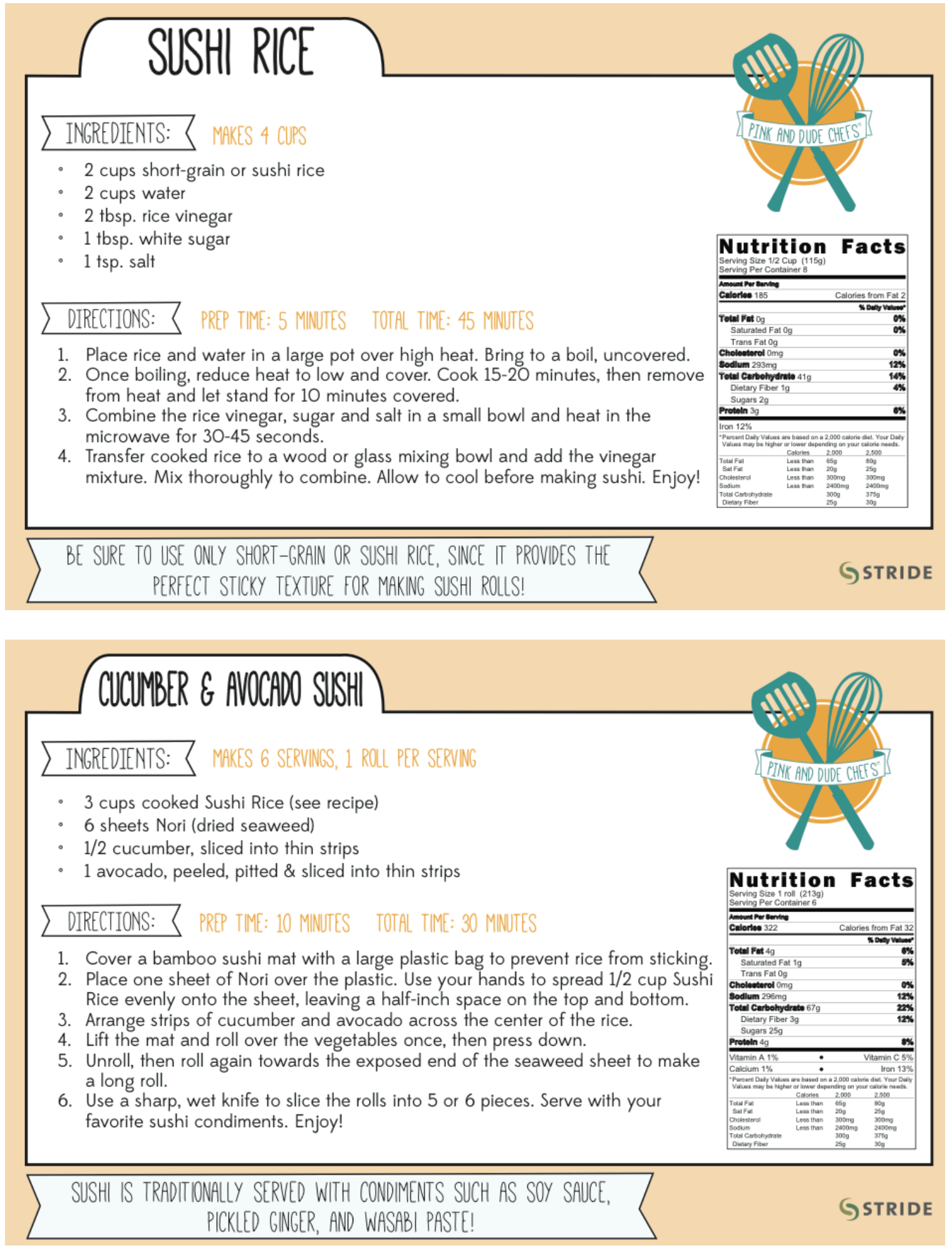

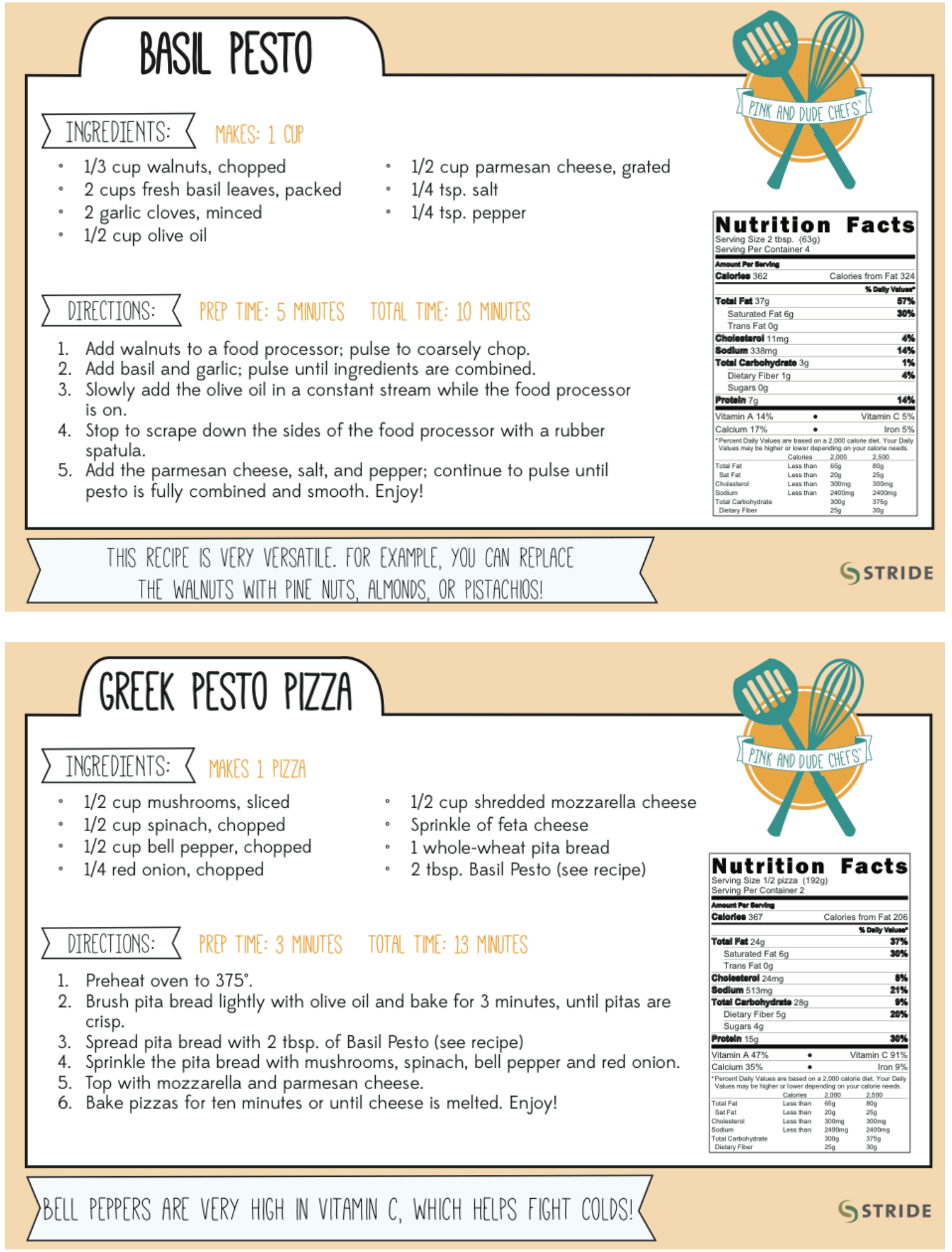


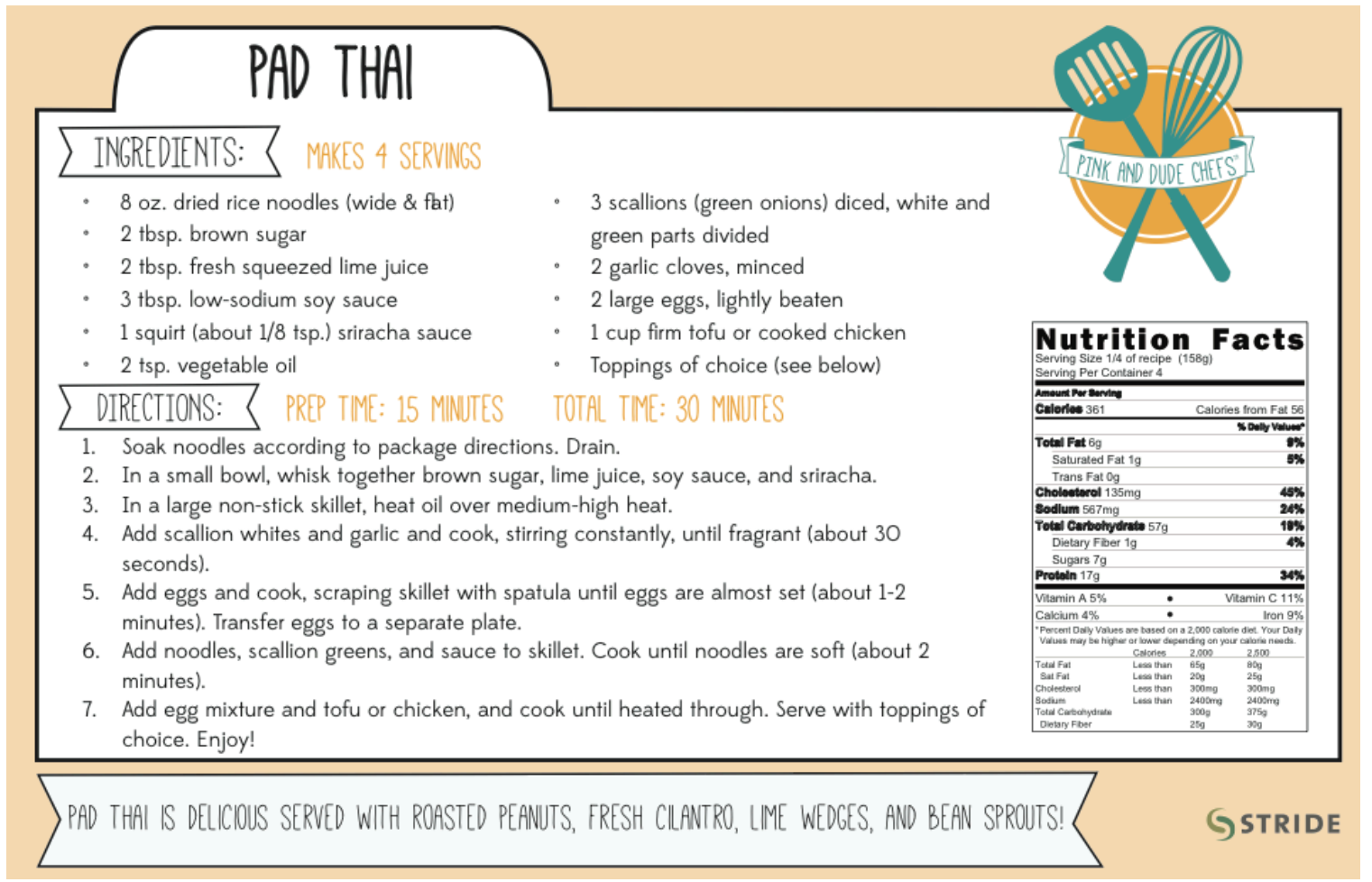

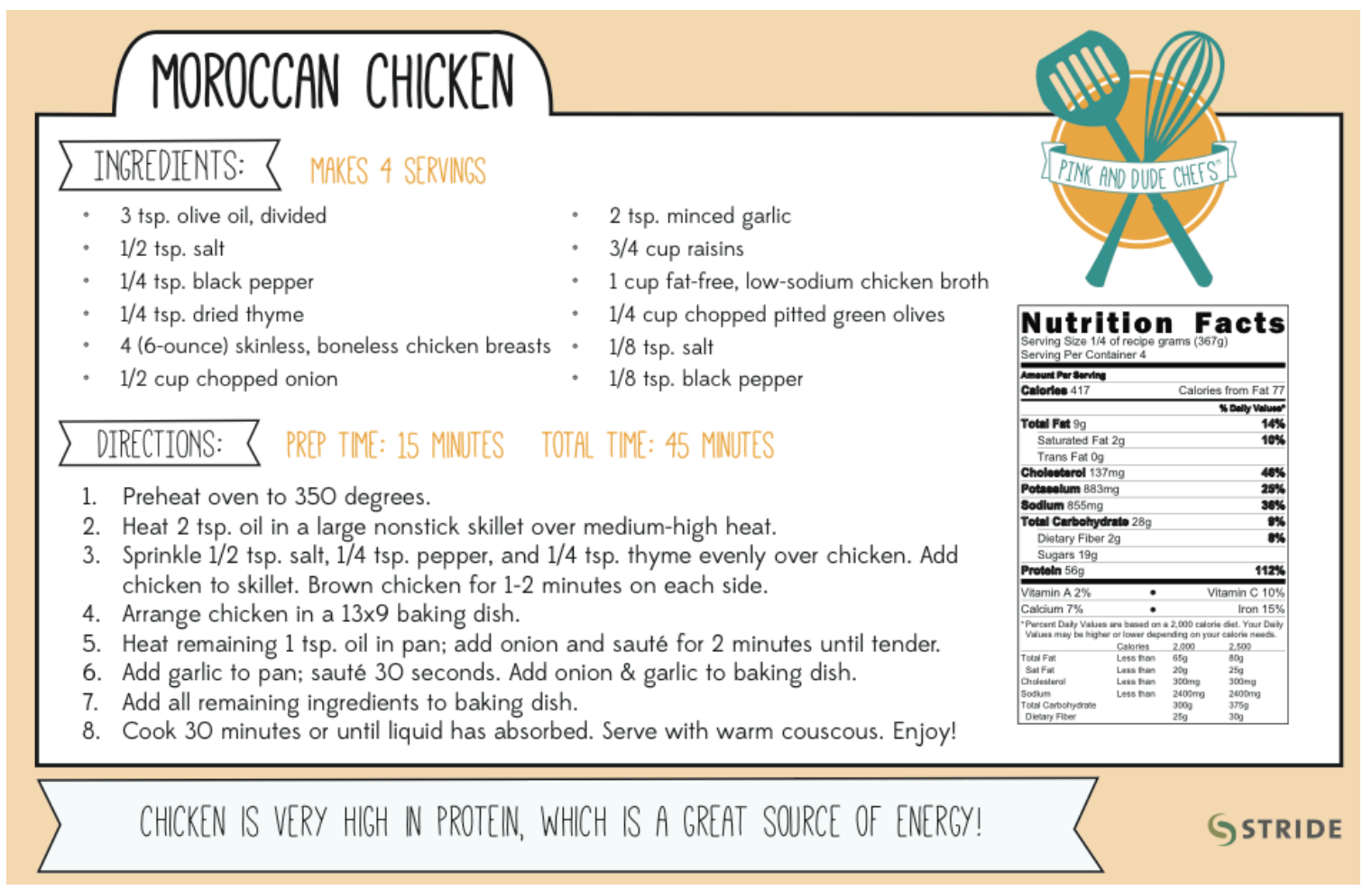




\title{
Appendix D: Human Subjects Protocol Approval Form
}

\author{
HUMAN SUBJECTS PROTOCOL APPROVAL FORM \\ Cal Poly, San Luis Obispo
}

All Cal Poly faculty, staff, and student research with human subjects, as well as ocher research imolving human subjects that is conduested at Cal Poly, must be reviewed by the Cal Poly Human Subjects Committee for the protection of human subjects, the researchers, and the University. Human subjects research is defined as any systematic investigation of living buman subjects that is designed to develop or contribute to generalizable knowledge. While the ethical gaidelines for research are applicable to classroom activities, demonstrations, and assigements, the Human Subjects Committee does not review classroom activities unless data will be collected and used in a sysuetratic investigation.

Researchers should complete all items on this approval form and submit three copies of it, along with three copies of a researts protocol (coetaining the informstion detailed is Curidelines for $\mathrm{H}$ man Subjects Reseirch Prowoeol), wo the Office of Research and Industry Relations (Debbic Hart, Bldg. 38, Room 154). Please feel free to attach an additional page if your responses to any of the items require more space. Your answers to the items on this form, as well as the reseirch protocol, sibald be typed. The Committee will make every effort no respood to your submission within two to four woeks. Committee approval should be received prior to contacting prospective subjects and collecting data. Please read carefully Cal Poly's Policy for the Use of Human Subjects in Research prior to completing this application

If you require assiztence in completing this form, contact the Office of Research and Indistry Relations at (805) 756-I508.

1. Date:

$$
\text { August 28, } 2013
$$

\section{Title of Research Project:}

Pink and Dude Chefs Phase 2: Evaluating the Impact of "Around the Worle" Curriculum
3. Type of Research:

\begin{tabular}{|l|l|}
\hline & Senior project \\
\hline $\mathrm{XX}$ & Master's thesis \\
\hline $\mathrm{X}$ & Faculty research \\
\hline $\mathrm{D}$ & Other: \\
\hline & please explain \\
\hline
\end{tabular}

4. Name(s) of Researcher(s)

Prineipal Investigator:

Department or other affiliation: $\quad$ Food Seience \& Nutrition

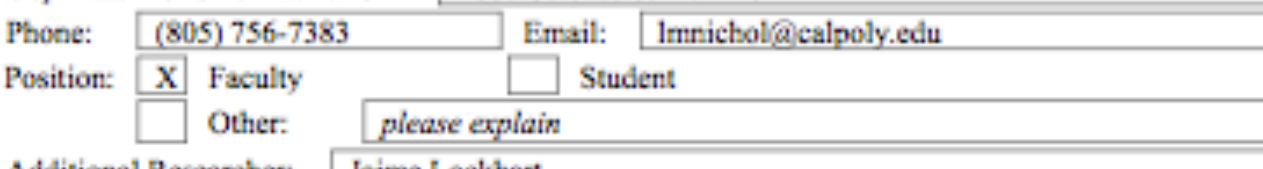

Additional Researcher: Jaime Lockhart

Department ot other affilistion: Food Seience \& Nutrition

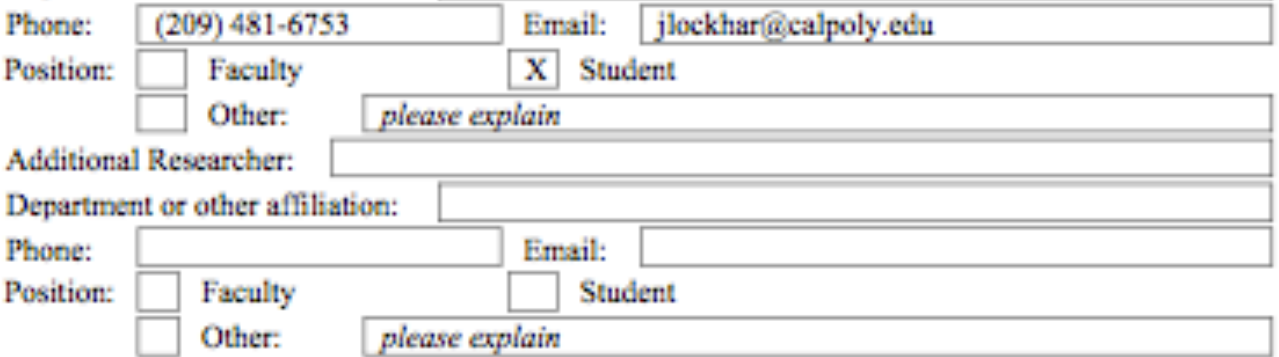

Any additional researchers involved in the project should be listed with the descriptive information requested above an a separate sheet. 
5. Faculty Advisor (if applicable)

Name: Dr. Lisa Nicholson Email: 1mnicbol@ealpoly.edu

\begin{tabular}{l|l|l|l|l|} 
Department or other affiliation: & Food Science \& Nutrition & Phone: (805) 756-7383
\end{tabular}

Other thesis committee members if the research is a thesis:

\begin{tabular}{|c|c|c|c|}
\hline Name: & Dr. Aydin Nazmi & Email: & nazmi@ealpoly.edu \\
\hline \multicolumn{2}{|c|}{ Department or other affiliation: } & Food Science \& Nutrition & Phone: (805) 756-6183 \\
\hline Name: & Dr. Arlene Grant-Holc & Email: & agrantbo@calpoly.edu \\
\hline \multicolumn{2}{|c|}{ Depsrtment or other affiliation: } & Food Science \& Nutrition & $(805) 756-5495$ \\
\hline Name: & & Email: & \\
\hline \multicolumn{2}{|c|}{ Department or other affiliation: } & & Phone: \\
\hline
\end{tabular}

6. Is there an external funding source for the project:

$\mathrm{X}$ Yes, and the source is: San Luis Oobispo Food Bank
No

7. Is this a modification of a project previously reviewed by Cal Poly's Human Subjects Committee?

$\mathrm{X}$ Yes, and the approximate date of the last review was: June 2011 No

8. Estimated duration of the project:

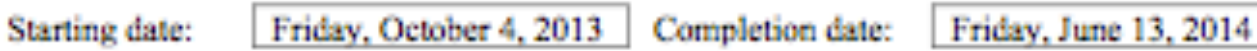

9. Describe any risks (physical, psychological, social, or economic) that may be involved.

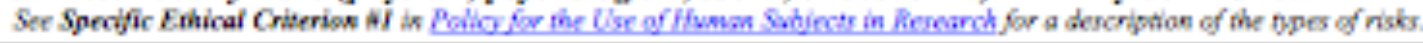

The possible risks associated with participation in this study are minor but may include burns from stove top, oven, scalding water, cuts from knives; falls from spillage; cboking from food consumption; or psychological stress from eompleting survey questions.

10. Indicate what measures will be taken to minimize risks. Sec Specific Euhicel Crinerion al in Poấig for

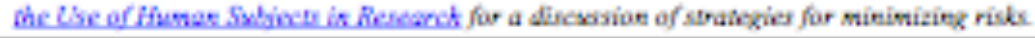

During food preparation, the subjects will be direetly supervised to reduce the risk of injury. The subjects will be reçuired to wear elosed-toed sboes with traction (slip-resistant sboes) for their protection. The subjects will be taught safety rules for extinguishing a fire (a fire extinguisher is located in the kitchen), proper knife use and storage, consistent use of potholders to reduce the risk of burns, food safety and the proper handling of food to prevent food-borne illnesses. Participants will be given contact information for the researchers to adéress any psycbological stress resulting from survey completion or participation.

11. Explain how subjects' confidentiality will be protected. See Specific Entical Criteriow w5 is Bolicy for the

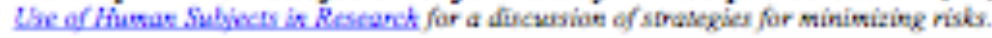


To protect the subjects' confidentiality, all identifying information will be kept in a filing eabinet in a locked room. Participants will only be identified by a code number on data sheets or other paperwork. Only project coordinators will have aceess to the identifying information. The subjects' responses will remain private and only presented as anonymous or group data.

12. Describe any incentives for participation that will be used. See Specific Estical Crixerian $2 z$ in Rolicy for

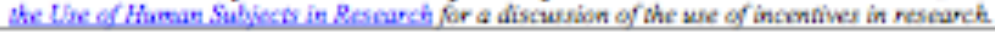

Incentives will inclade: cooking tools and supplies, which are given for atterdarce and participation in activities, games, and çuestionnaires. In addition, participants will be given the foods prepared in class to take home.

13. Will deception of subjects be involved in the research procedures? Yes* $\mathrm{X}$ No

"If so, explain the deception and how it will be handled. See Specifie Entical Criterian *3 in Folicy for the Use

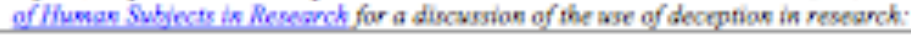

14. Type of review requested:

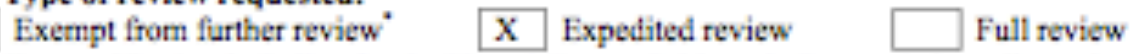

See Types of Review in Policy for the Use of Human Subjects in Research for a discussion of the criteria for exempt, expecited, and full reviews.

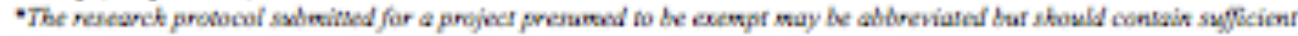

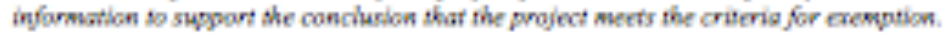

\section{Sipnatures:}

Your signature below irdicates that the information presented in this applieation (the approval form and research protocol) is aecurate and that you have read, understard, and agree to follow the Policy for the Use of Human Subjects in Research.

Name of Primary Researcher: Jaime Lockhart

Signature:

Cal Poly Faculty Advisor's Signature (Required if this is student research)

I have reviewed this research proposal which has been prepared by my actvisee(s) in accoedarce with the Guicelines for Obtaining Human Subjects. Approval.

Name of Faculty Advisor:

Lisa M. Nicholson

Signature 


\title{
Appendix E: Human Subjects Research Protocol
}

\author{
Human Subject Research Protocol
}

Title

Pink and Dude Chefs Phase 2: Evaluating the Impact of "Around the World" Curriculum

Faculty and Department

Lisa Nicholson, PhD, RD. Professor, Food Science and Nutrition Department

\section{Statement of Purpose}

Based on the Social Cognitive Theory (SCT) and Kolb's Model of Experiential Learning, this afterschool program expands on the pilot study done in 2008 and a formative research project completed in 2013 by other Nutrition graduate students. The key questions of interest are whether teaching middle-school-aged children cooking skills, along with basic food and nutrition information, will increase confidence for cooking and whether the increased confidence influences self-regulation of food and nutrition related choices. The previous formative work suggests that the increased knowledge and skills does increase confidence but that many other factors, including social norms, also influence a middle school-aged child's food choices. The current proposal will research the impact of Phase 2 of Pink \& Dude Chefs, called "Around the World." The Phase 2 curriculum explores the culture and cuisine of a different country each week while still focusing on building cooking skills and nutrition knowledge. Additionally, the use of weekly class-related goals designed to be completed between classes will measure selfregulation and the influence of goal attainment on social norms. We hope to test whether Phase 2 increases culinary self-efficacy and nutrition knowledge and whether the Phase 2 design changes family \& social norms associated with cooking.

\section{Methods}

Subjects: The current project will take place at Mesa Middle School, 2555 Halcyon Road, Arroyo Grande. Subjects will be drawn from students who are signing up for the Bright Futures afterschool program. Both boys and girls, ages 11-14 years, are welcome in the Bright Futures program. Students will need to have private transportation to the locations after school which may limit participation. We intend to run the program in each of the Cal Poly quarters with Jaime Lockhart as project supervisor, supervisory responsibilities include maintaining quality control and adherence to research protocols with the work of the Cal Poly Health Ambassadors class who will be teaching and assisting in the weekly cooking classes. Children do constitute a vulnerable group, therefore, parents' consent for participation will be sought. Research assents will also be attained from these middle-school participants.

Experimenter: Dr. Lisa Nicholson will be the primary investigator on this study. Dr. Nicholson is a Registered Dietitian and has her doctorate in Health Behavior Research in Preventative Medicine. In addition to her teaching in the Nutrition program she has researched, published and given talks on tailoring nutrition education approaches to match specific audiences. She has experience creating nutrition curricula for children and young teens.

Jaime Lockhart, the individual administering the study, will be receiving a Master's in Agriculture with a specialization in Food Science and Nutrition. The experimenter received a Bachelor of Arts degree in Geography at University of California, Santa Barbara. The experimenter is currently the STRIDE Health Ambassador Program coordinator after having completed two quarters as an enrolled student in KINE 471-03 Health Ambassadors, one quarter

Pink \& Dude Chefs: Phase 2 - Human Subjects Research Protocol. 8.28.13 
as a STRIDE Health Ambassador intern, and one quarter as the STRIDE Health Ambassador Program co-coordinator. These experiences provided the essential knowledge for implementing a community-based cooking course: practical cooking classes, meal planning and budgeting, community involvement, classroom control, lesson planning, and knowledge of the USDA Food Guide "My Plate" recommendations, etc.

\section{Materials \& Procedures:}

Instruments: Copies of the questionnaires can be found at the end of this protocol. There will be a written survey given to the Bright Future students at the beginning of the first week of each program and at the last week of the final session. In addition, students will be asked to participate in a one-hour focus group at the end of the each session to determine attitudes regarding the program, cooking, and health. These focus group questions follow the Kirkpatrick method of evaluating trainings and workshops.

Locations: The program will take place at Mesa Middle School, 2555 Halcyon Road, Arroyo Grande, California.

Chronological description of procedures: Each Pink and Dude Chef Program consists of a twohour class held once weekly for eight weeks. These eight classes each start with a didactic component that teaches specific cooking skills with a focus on incorporating fruits and vegetables into recipes. The didactic component primarily focuses on familiarizing middleschool participants with basic cooking skills, food and nutrition knowledge, and cultural foodways. Weekly active discussion of time management and goal setting is incorporated into the initial didactic part of the session. Additional content area includes web-based food, nutrition, and cooking resources. The majority of time in each class is spent on hands on learning and practicing the targeted cooking skills to create the recipes for the day. The San Luis Obispo Food Bank will be donating foods to the program based on weekly availability. Cal Poly students enrolled in KINE 471-03 Health Ambassadors class and STRIDE volunteers will be trained to teach, assist, and supervise the cooking classes. We anticipate at least one Cal Poly student per two to three afterschool participants. Cal Poly students will in turn be supervised by Dr. Lisa Nicholson and Jaime Lockhart, the STRIDE Health Ambassador Program coordinator.

\section{Informed Consent}

Please see attached informed consent form for the parents or guardians of potential participants. 


\title{
Appendix F: Informed Assent Form for Participant
}

\author{
Informed Assent Form for Cal Poly STRIDE Research \\ INFORMED ASSENT TO PARTICIPATE IN: \\ Pink and Dude Chefs, a program of Cal Poly STRIDE \\ For the Participant
}

Pink and Dude Chefs is a research project that was created by professors and students at Cal Poly, San Luis Obispo. The people who are currently running the project are Dr. Lisa Nicholson in the Food Science and Nutrition Department, and Jaime Lockhart, a graduate student in the Food Science and Nutrition Department at Cal Poly, San Luis Obispo, California. The goal of Pink and Dude Chefs is to use cooking classes to make you more confident in your ability to cook meals at home. We hope to do this by teaching you the skills to prepare food, showing you how to buy food, and allowing you to create a menu.

Pink and Dude Chefs has a total of 8 educational cooking classes: one class per week on Tuesdays. These classes will include a short talk regarding the daily topic; group activities and games; and cooking the recipe of the day. The classes are held at Mesa Middle School, 2555 Halcyon Road, Arroyo Grande, California. Cal Poly STRIDE will not be able to provide you with rides to or from Mesa Middle School.

As part of Pink and Dude Chefs, we will be asking you to take a survey at the beginning and end of the 8 -week program. The questions in the survey will ask you about what you usually eat, how you feel about cooking, how you rate your cooking skills, and basic nutrition questions.

There will also be a 1- to 2-hour focus group at the end of the 8-week program. Focus groups are a chance for you to tell us how you felt about the program and for us to ask you more questions about your experiences with the program. During the focus group, Jaime Lockhart, the STRIDE Health Ambassador Coordinator, will lead a discussion with you on how you feel about cooking, diet, and exercise. This group discussion will be audio recorded.

You are not required to take the survey, go to the focus group, or take the questionnaire home and you can stop coming to the Pink and Dude Chefs classes at any time. You can also skip any questions you prefer not to answer.

There will always be adults present to prevent injuries during cooking and activities. You'll be required to wear closed-toed shoes with traction (slip-resistant shoes) for your protection. You will be taught safety rules for extinguishing a fire (a fire extinguisher is located in the kitchen), proper knife use and storage, consistent use of pot holders to reduce the risk of burns, food safety and the proper handling of food to prevent food-borne illnesses.

There is a chance you may be injured as a result of participating in this program. The possible risks may include: burns from stovetop, oven, scalding water, cuts from knives, falls from spillage, choking from food consumption, or psychological stress from completing survey questions. If you get hurt while participating in Pink and Dude Chefs activities, notify an adult in the room immediately. Your parents have been told how to get medical attention if you require it. If you're uncomfortable with any of the questions or activities, please contact Jaime Lockhart, Program Coordinator for Pink and Dude Chefs at (209) 481-6753 for assistance.

The surveys that you take are all confidential. This means that we will write a code number on your survey instead of your name. Any information about you will be kept in a filing cabinet in a locked room. We will use a code number on any data sheets or other paperwork instead of your name. Only project coordinators will have access to any information about you. Your answers will remain private and only presented as anonymous or group results.

1 out of 2 
There are benefits that you might gain from participating in this program. These include: increased knowledge of nutrition and web-based cooking resources, building skills for healthful cooking, communication, time management and goal setting, and increased confidence for cooking family meals. You will be able to bring food home weekly. Other incentives you might receive include cooking tools and supplies, which are given for attendance and participation in activities and games.

We would like to take photographs and video of you as you participate in the program and use these images in presentations and publicity. Please indicate below if you don't want us to take photographs or video of you. You will not be identified by name.

NO, I do not give permission for my photo and video to be taken for presentations and media use.

If you want to participate in this research project as described, please indicate this by signing below. Please keep one copy of this form for your parent/guardian.

Thank you for your participation in this study!

$\begin{array}{ll}\text { (PRINT) Name of Child Volunteer } & \text { Date }\end{array}$

(SIGNATURE) Name of Child Volunteer 


\title{
Appendix G: Informed Permission Form for Parents
}

\author{
Informed Consent Form for Cal Poly STRIDE Research \\ INFORMED PERMISSION TO PARTICIPATE IN: \\ Pink and Dude Chefs, a program of Cal Poly STRIDE
}

A research project using cooking classes to increase confidence for cooking meals prepared at home is being conducted by Dr. Lisa Nicholson in the Food Science and Nutrition Department and Jaime Lockhart, a graduate student in the Food Science and Nutrition Department at Cal Poly, San Luis Obispo, Califormia. The purpose of this study is to improve your child's confidence for cooking and to teach your child menu planning, food buying and food preparation skills.

Your child is being asked to take part in this study by attending a total of 8 educational cooking elasses: one 2-hour class per week held on Tuesdays. These classes will include a short talk regarding the daily topie; group activities and games; and cooking the recipe of the day. The program will take place at Mesa Middle School, 2555 Halcyon Road, Arroyo Grande, California. No transportation to or from Mesa Middle School will be provided by our program.

Your child will be asked to complete a survey before and a survey after the 8-week program. These questions ask about your child's current diet, attitudes towards cooking, cooking skills, and general nutrition knowledge.

There will also be a 1- to 2-hour focus group following the end of the 8-week program. During this focus group, Jaime Lockhart, the STRIDE Health Ambassador Coordinator, will lead a discussion with the children about their attitudes towards cooking, diet, and exercise. These group discussions will be audio recorded.

Please be aware that your child is not required to participate in this research and your child may discontinue his/her participation at any time. Your child may also omit any questions he/she prefers not to answer.

During food preparation, your child will be directly supervised to reduce the risk of injury. Your child will be required to wear closed-toed shoes with traction (slip-resistant shoes) for their protection. Your child will be taught safety rules for extinguishing a fire (a fire extinguisher is located in the kitchen), proper knife use and storage, consistent use of pot holders to reduce the risk of burns, food safety and the proper handling of food to prevent food-borne illnesses.

The possible risks associated with participation in this study are minor but may include burns from stovetop, oven, scalding water, cuts from knives, falls from spillage, choking from food consumption, or psychological stress from completing survey questions. If your child should experience any injuries due to possible cuts or burns, please be aware that you may contact your own physician/clinic or the Arroyo Grande Fire Department at 2391 Willow Rd., Arroyo Grande (805) 473-7171. Insurance coverage is not offered for this program, should your child experience any injuries due to participation in the classes. If you should experience any discomfort with any part of the study, please be aware that you may contact Jaime Lockhart, Program Coordinator for Pink and Dude Chefs (209) 481-6753 for assistance.

To protect your child's privacy all identifying information will be kept in a filing cabinet in a locked room. Participants will only be identified by code number on data sheets or other paperwork. Only project coordinators will have access to the information. Your child's responses will remain private and only presented as anonymous or group data.

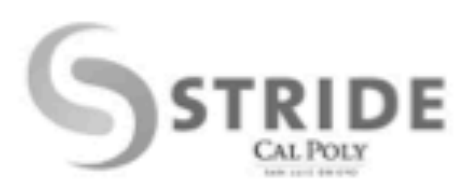


Potential benefits associated with this program include: increased knowledge of nutrition and webbased cooking resources, building skills for healthful cooking, communication, time management and goal setting, and increased confidence for cooking family meals. All participants will bring food home weekly. Other incentives your child may receive include cooking tools and supplies, which are given for attendance and participation in activities and games.

We would like to take photographs and video of the children as they participate in the program and use these images in presentations and publicity. Please indicate below if you do not authorize this. Your child will not be identified by name.

NO, I do not give permission for my child's photo and video to be taken for presentations and media use.

If you have questions regarding this study or would like to be informed of the results when the study is completed, please feel free to contact Dr. Lisa Nicholson at (805) 756-7383 and/or the STRIDE office (805) 756-0673, STRIDE@calpoly.edu. If you have concerns regarding the manner in which the study is conducted, you may contact Dr. Steve Davis, Chair of the Cal Poly Human Subjects Committee, at (805)756-

2754, sdavis@calpoly.edu or Dr. Dean Wendt, Interim Dean of Research at (805) 756-2988

ordwendt@calpoly.edu. 